\title{
Discovery of new members of the nearby young stellar association in Cepheus $\star, \star \star$
}

\author{
A. Klutsch ${ }^{1,2,4}$, A. Frasca ${ }^{2}$, P. Guillout ${ }^{3}$, D. Montes ${ }^{4}$, F.-X. Pineau ${ }^{3}$, N. Grosso ${ }^{5}$, and B. Stelzer ${ }^{1,6}$ \\ ${ }^{1}$ Institut für Astronomie und Astrophysik, Eberhard Karls Universität, Sand 1, 72076 Tübingen, Germany \\ e-mail: klutsch@astro.uni-tuebingen. de \\ 2 INAF-Osservatorio Astrofisico di Catania, Via S. Sofia 78, 95123 Catania, Italy \\ 3 Observatoire Astronomique, Université de Strasbourg \& CNRS, UMR 7550, 11 Rue de l'Université, 67000 Strasbourg, France \\ ${ }^{4}$ Departamento de Física de la Tierra y Astrofísica and IPARCOS-UCM (Instituto de Física de Partículas y del Cosmos de la UCM), \\ Facultad de Ciencias Físicas, Universidad Complutense de Madrid, 28040 Madrid, Spain \\ 5 Aix Marseille Univ, CNRS, CNES, LAM, Marseille, France \\ 6 INAF-Osservatorio Astronomico di Palermo, Piazza del Parlamento 1, 90134 Palermo, Italy
}

Received 29 November 2019 / Accepted 7 March 2020

\begin{abstract}
Context. Young field stars are hardly distinguishable from older ones because their space motion rapidly mixes them with the stellar population of the Galactic plane. Nevertheless, a careful target selection allows for young stars to be spotted throughout the sky. Aims. We aim to identify additional sources associated with the four young comoving stars that we discovered towards the CO Cepheus void and to provide a comprehensive view of the Cepheus association.

Methods. Based on multivariate analysis methods, we have built an extended sample of 193 young star candidates, which are the optical and infrared counterparts of ROSAT All-Sky Survey and XMM-Newton X-ray sources. From optical spectroscopic observations, we measured their radial velocity with the cross-correlation technique. We derived their atmospheric parameters and projected rotational velocity with the code ROTFIT. We applied the subtraction of inactive templates to measure the lithium equivalent width, from which we infer their lithium abundance and age. Finally, we studied their kinematics using the second Gaia data release.

Results. Our sample is mainly composed of young or active stars and multiple systems. We identify two distinct populations of young stars that are spatially and kinematically separated. Those with an age between 100 and $300 \mathrm{Myr}$ are mostly projected towards the Galactic plane. In contrast, 23 of the 37 sources younger than $30 \mathrm{Myr}$ are located in the CO Cepheus void, and 21 of them belong to the stellar kinematic group that we previously reported in this sky area. We report a total of 32 bona fide members and nine candidates for this nearby (distance $=157 \pm 10 \mathrm{pc}$ ) young (age $=10-20 \mathrm{Myr}$ ) stellar association. According to the spatial distribution of its members, the original cluster is already dispersed and partially mixed with the local population of the Galactic plane.
\end{abstract}

Key words. stars: pre-main sequence - stars: fundamental parameters - stars: kinematics and dynamics - X-rays: stars

\section{Introduction}

The "natural" birth sites of stars are young open clusters and starforming regions (SFRs) which are often tightly associated with emission nebulae and molecular clouds (e.g., Piskunov et al. 2008; Zinnecker 2008). However young stars are also found in wide regions around SFRs and also in the field, apparently unrelated to any of the known star-forming sites. Such populations of young stars are composed of both classical T Tauri stars (CTTSs) and weak-line T Tauri stars (WTTSs). There is evidence of different space distributions for CTTSs and WTTSs, the former

\footnotetext{
* Tables 1, 3-5 are only available at the CDS via anonymous ftp to cdsarc.u-strasbg. fr $(130.79 .128 .5)$ or via http://cdsarc. u-strasbg.fr/viz-bin/cat/J/A+A/637/A43

$\star \star$ Based on observations collected at the Isaac Newton Telescope (INT) operated on the island of La Palma by the Isaac Newton Group in the Spanish Observatorio del Roque de Los Muchachos of the Instituto de Astrofísica de Canarias, the $2.2 \mathrm{~m}$ telescope of the GermanSpanish Astronomical Centre, Calar Alto (Almería, Spain), operated by the Max-Planck-Institute for Astronomy, Heidelberg, jointly with the Spanish National Commission for Astronomy, and the SOPHIE spectrograph on the $1.93 \mathrm{~m}$ telescope at Observatoire de Haute-Provence (CNRS), France (program 09A.PNPS.GUIL).
}

located near the cloud cores while the latter are spread all around the SFR (e.g., Alcala et al. 1997). Moreover, WTTSs are on average older than CTTSs and have already dissipated their accretion disks (Bertout et al. 2007). These young stars may form substructures that can be located up to tens of parsecs away from the SFR's core (e.g., Mapelli et al. 2015).

In the 1990s, various scenarios for explaining the presence of dispersed young stars were proposed. For those located in the outskirts of SFRs or in the space surrounding them, the simplest explanation was that they had drifted by thermal velocity dispersion of gas within star-forming sites. On the other hand, those far away from any conventional SFRs should have formed locally from cloudlets in turbulent giant molecular clouds (Feigelson 1996). However neither of these theories could explain the presence of T Tauri stars (TTSs) with high space velocity, the so-called "runaway" TTSs (Sterzik et al. 1995). Their ejection could have occurred during the dynamical evolution of young multiple systems and come from close encounters with other members of their parent cloud (Sterzik \& Durisen 1995, 1998; Gorti \& Bhatt 1996).

The picture of star formation in our Galaxy is still not welldefined and new important details are being added by the recent 
large spectroscopic surveys and astrometric space missions, such as Gaia. One of the most relevant results of the Gaia-ESO survey (Gilmore et al. 2012; Randich et al. 2013) is the discovery of two kinematically distinct populations (A and B) in the field of both the Gamma Velorum (Jeffries et al. 2014) and NGC 2547 (Sacco et al. 2015) clusters. The properties of sources belonging to Gamma Velorum B and NGC 2547 B are fairly similar in terms of age and kinematics. Since these two clusters are close to each other $\left(\approx 6^{\circ}\right.$ on the sky corresponding to about $40 \mathrm{pc}$ at their distance) and both are in the field of the Vela OB2 association, the $\mathrm{B}$ populations of these two clusters might form an extended low-mass population in the Vela OB2 association.

Over the past two decades, several nearby $(30-150 \mathrm{pc})$ young (5-70 Myr) associations were identified, mostly in the southern hemisphere (Zuckerman \& Song 2004). Torres et al. (2006, 2008) found many of them and their members during the SACY survey. The use of a Bayesian analysis and, subsequently, the BANYAN $\Sigma$ multivariate Bayesian algorithm contributed significantly to the continued identification of new members, mainly low-mass stars and brown dwarfs (Malo et al. 2013; Gagné et al. 2014, 2015a,b, 2018a). The first release of the Gaia mission (Gaia Collaboration 2016) improved the astrometric accuracy for the Tycho sources. This has made it possible to search for new comoving stars (Oh et al. 2017), as well as stellar kinematic groups and clusters (Faherty et al. 2018; Gagné et al. 2018b). Based on the very accurate astrometry from the second data release of the Gaia mission (Gaia DR2, Gaia Collaboration 2018), Gagné \& Faherty (2018) discovered a considerable number of likely new members in these young associations.

However, it remains difficult to recognize the young stars without a circumstellar disk in the field among the Galactic plane stellar population. Neither their global photometric properties nor the presence of nearby gas differentiates them from older stars. An efficient methodology for identifying the young stars is through the use of large X-ray surveys because the stellar $\mathrm{X}$-ray sources in the ROSAT catalog are mainly stars younger than 1 Gyr (e.g., Motch et al. 1997a). Guillout et al. (1998a) showed that this stellar population can be used as a tracer of young local structures, like the late-type stellar population in the Gould Belt (Guillout et al. 1998b). To this end, Guillout et al. (1999) cross-correlated the ROSAT All-Sky Survey (RASS) with the Tycho catalog building the first large dataset $(\approx 14000$ objects) of late-type stellar X-ray sources, the so-called RasTyc sample.

Spectroscopic surveys of northern RasTyc sources were performed by Guillout et al. (2009, Paper I) and Frasca et al. (2018, Paper III). In Paper I, we identified five young field stars in the optically bright $(V<9.5 \mathrm{mag})$ sample. Afterwards, $\mathrm{BD}+443670$ and $\mathrm{BD}+45598$ were recognized as members of the Columba association ( 30 Myr; Zuckerman et al. 2011) and of the $\beta$ Pic moving group ( $\sim 12$ Myr; Moór et al. 2011), respectively. Klutsch (2008) noticed that an almost uniform spatial density of young stars in the optically bright RasTyc sample. At the same time, the spatial density in the northern hemisphere (Paper I) is about one order of magnitude lower, on average, than that in the southern hemisphere (the SACY survey). This is consistent with the significant asymmetry in the all-sky RasTyc map with respect to the Galactic plane, as reported by Guillout et al. (1998a), and is likely related to the structure of the Gould Belt. A higher fraction of young stars was found in the optically faint sample (Paper III). This may be due to the larger distances involved, on average, in the faint survey, which facilitated the detection of many more intrinsically brighter $\mathrm{X}$-ray (more active and younger) sources when compared to the bright sample. A larger contribution from the Gould Belt, which is more distant in the northern hemisphere, can also explain these differences.

Klutsch (2008) analyzed the early spectroscopic observations of optically faint RasTyc sources. This led to the discovery of an over-density of stellar X-ray sources near the Cepheus-Cassiopeia complex. We refer the reader to the reviews on SFRs in the Cassiopeia and Cepheus constellations of Kun (2008) and Kun et al. (2008), respectively. Although this sky area is rich in CO molecular regions (Dame et al. 2001) and dark clouds (Dobashi et al. 2005; Kiss et al. 2006), Guillout et al. (2010, Paper II) identified four comoving TTSs towards a region devoid of interstellar matter that is denoted as the fourth void in the Cepheus Flare region by Kiss et al. (2006). Moreover, Tachihara et al. (2005) had already reported the discovery of 16 WTTSs in this region. In Paper III, we found four other likely members of this group based on their spectral characteristics, position in the HR diagram, and kinematic properties. The group 38 in Oh et al. (2017) and Faherty et al. (2018) is composed of seven stars all belonging to the young association analyzed in the present paper and formerly discovered by our group (Klutsch 2008; Paper II), which already included four of their seven stars; here, we extend the census of this young association.

The main aim of the present work is to identify other young stars surrounding the four comoving TTSs in Cepheus reported in Paper II, and to characterize their physical and kinematic properties, including the WTTSs provided by Tachihara et al. (2005). In Sect. 2 we detail our procedure to build a sample of "young star candidates", based on the data available in 2009, when it was created. We also describe our campaign of optical spectroscopic observations. Section 3 presents our analytical methods and results thereof. In Sect. 4 we mainly focus the discussion on the properties of the selected sources, on the spatial distribution and kinematics of the young stars belonging to the young association towards the CO Cepheus void, and on its reliability. Finally, we outline our conclusions and perspectives in Sect. 5. We present the extraction of spectra with a double Gaussian profile in Appendix A. We also provide further information on multiple systems (Appendix B), low-mass stars (Appendix C), and a few specific targets (Appendix D).

\section{Sample and observations}

\subsection{Sample selection}

The greatest difficulty in finding young stars in the field is the selection of a suitable sample of candidates. The best manner for identifying such a population is to excavate into an extensive and comprehensive sample of stellar X-ray sources, such as the RasTyc sample (Guillout et al. 1999, Papers I and III).

To this end, we picked out all counterparts of the X-ray sources by cross-matching the RASS catalog (Voges et al. 1999, 2000) with the Two Micron All-Sky Survey catalog (2MASS, Skrutskie et al. 2006) using a likelihood ratio approach and an original way to estimate the rate of spurious associations (Pineau et al. 2008a,b, 2011; Pineau 2009). We discarded all the matches having an angular separation larger than $15^{\prime \prime}$ and a probability of identification less than 0.7 . We then correlated the remaining sources with the Guide Star Catalog II (GSC II, Lasker et al. 2008). We kept all the optical sources at an angular distance not exceeding $5^{\prime \prime}$ from 2 MASS sources and $15^{\prime \prime}$ from RASS ones.

We proceeded in a similar way for the XMM-Newton X-ray sources of the 2XMMi catalog (Watson et al. 2009). This turned 


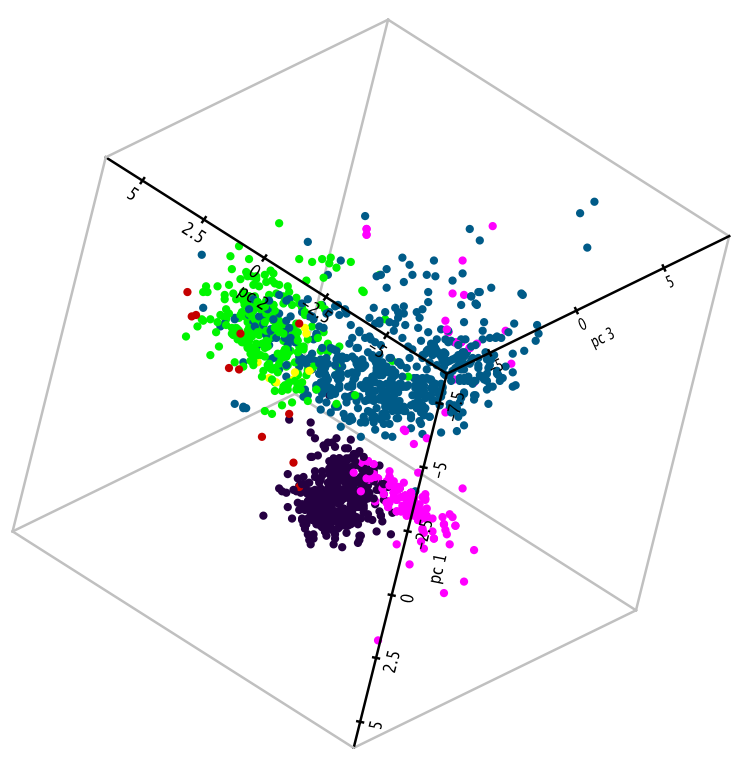

Fig. 1. Result of the principal component analysis applied to RASS X-ray sources. The 3D scatter plot displays the three first components, adopting different colors for quasars (green dots), galaxies (blue dots), and stars (other dots). Such a method optimizes the disentangling of various classes of X-ray emitters. The pink dots denote the locus of a priori young stars selected using our learning sample (see text).

out to be quite inconclusive because we only selected three stars. However, we note that the source 1RXS 184257 (=[KP93] 2-43 in Kun \& Prusti 1993), which is a well-known young visual binary, is part of the selection. That makes us confident in the effectiveness of our approach, even for optically faint sources.

We then applied a multivariate analysis on these two X-ray selected datasets. A principal component analysis, which takes measurements errors (Pineau et al. 2008b) on 19 parameters into account, was used to build up linear combinations of flux ratios from X-ray to near-infrared (NIR) wavelength domain, X-ray hardness ratios, and color indexes. Applying a mean shift procedure on the three first principal components, we were able to disentangle the stellar population from the extragalactic component (galaxies and quasars) that also emits X-rays (Fig. 1). We followed the guidelines of Pineau et al. (2011) to identify the local maximum of the population of well-known young stars in this three-dimensional space. We therefore built a reliable learning sample composed of sources whose object type is either young stellar object, T Tau-type star, or pre-main-sequence star in the Simbad database. We finally labeled all the likely stellar X-ray sources located in the latter region as our young star candidates. The objects are distributed all over the sky.

This work is focused on the identification of young stars towards the CO Cepheus void and its surroundings. Therefore, we selected all the young stars located in a $30^{\circ}$-wide region encompassing the four comoving TTSs discovered in Paper II in that region and we then restricted our sample to the population of late-type $(B-V>0.5 \mathrm{mag})$ and faint $(V>9 \mathrm{mag})$ stars. Relying on the photometric distance estimate of the group (Paper II), we retain all the candidates within $170 \mathrm{pc}$ of the Sun and with X-ray luminosity $L_{X} \geq 10^{29} \mathrm{erg} \mathrm{s}^{-1}$ (Guillout et al. 1998b). This $\mathrm{X}$-ray emission threshold picks the majority of 1 Myr-old stars with spectral type $G$ and K (Preibisch et al. 2005). The choice of these selection criteria and of this vast sky area allows us (i) to look for additional bona fide members both in a restricted region around the Cepheus void and far away (i.e., to search for any runaway WTTSs), and (ii) to characterize the population of stellar X-ray sources in an under-researched sky area. The 162 strongest stellar X-ray sources, which are also a priori the youngest stars selected, form our list of prime targets.

The low-resolution spectroscopic survey by Tachihara et al. (2005) includes 14 sources common to our sample, as well as one analyzed in Paper II (i.e., TYC 4500-1478-1). We decided to add the remaining 31 stars from Tachihara et al. (2005) to our sample, which leads to a total of 193 candidates. Table 1 lists their basic data, together with that of the eight young stars reported in Papers II and III (labeled as G1-G4 and F1-F4, respectively).

\subsection{Photometric and astrometric data}

The selection of young star candidates to be observed spectroscopically was performed before the launch of Gaia. At that time, only two of the 193 pre-selected candidates had reliable HIPPARCOS parallaxes, while 102 sources, which are included in the Tycho catalog (ESA 1997), had unreliable parallaxes and proper motions with a fair accuracy. We therefore needed multiband photometric data (from optical to infrared wavelengths) to select the young star candidates with the multivariate analysis described in Sect. 2.1. However, meanwhile, the Gaia DR2 catalog reports very accurate parallaxes and proper motions for nearly all the sources investigated in the present paper, which allows us to study their kinematics and evolutionary status.

Since we only selected stellar X-ray sources with a 2MASS counterpart, their photometry in the NIR wavelength domain is homogeneous. The $J, H$, and $K_{s}$ magnitudes of the targets are in the range 7-12 mag and have generally a high level of accuracy (in more than $96 \%$ of cases). For seven sources, at least one of NIR magnitudes is an upper limit or a poor-quality value in the final release of 2MASS data.

We encountered difficulties with the homogenization of photometry in optical bands. Indeed, when we started our target selection, no catalog provided Johnson $B$ and $V$ magnitudes up to $V=14.5 \mathrm{mag}$. The completeness of the Tycho-2 cata$\log (\mathrm{H} \varnothing \mathrm{g}$ et al. 2000) is to about $90 \%$ for sources brighter than $V \sim 11.5 \mathrm{mag}$, although it also contains fainter stars. For the nonTycho sources fainter than $V=12 \mathrm{mag}$, only the photographic magnitudes were available and reliable. For the 102 sources with an entry in the Tycho- 2 catalog, the $V_{T}$ magnitude ranges from 9 to $13 \mathrm{mag}$ (Fig. 2). We converted the $B_{T}$ and $V_{T}$ magnitudes into Johnson $B$ and $V$ magnitudes following the transformation given in the introduction of the Tycho-2 catalog: $B=B_{T}-0.24\left(B_{T}-V_{T}\right)$ and $V=V_{T}-0.09\left(B_{T}-V_{T}\right)$.

For 79 of the remaining sources with both $B_{J}$ and $R_{F}$ photographic magnitudes in the USNO-B1.0 catalog (Monet et al. 2003), we estimated the $V$ magnitude using the formula proposed by Lépine \& Shara (2005): $V=B_{J}-0.46\left(B_{J}-R_{F}\right)$. We used the $B_{J}$ and $R_{F}$ magnitudes extracted from the second Palomar Observatory Sky Survey (POSS) scans in the IIIa-J and IIIa-F passbands, respectively. All these sources are fainter than $V=11$ mag. Additional magnitudes in the Johnson $B$ band were retrieved either from the GSC II catalog or from the unpublished Yellow-Blue 6 catalog (YB6 - USNO). The latter is available through the NOMAD catalog (Zacharias et al. 2004).

In Table 1 , we mainly list the $B$ and $V$ magnitudes in the Johnson-Cousins system ( $\sim 88 \%$ and $\sim 94 \%$ of sources). If one of these values are not available, we substitute them by the $B_{J}$ or $V$ photographic passbands taken from GSC II (Fig. 2).

However, for the analysis of the spectral energy distribution (Sect. 3.3) we have updated the magnitudes with more 

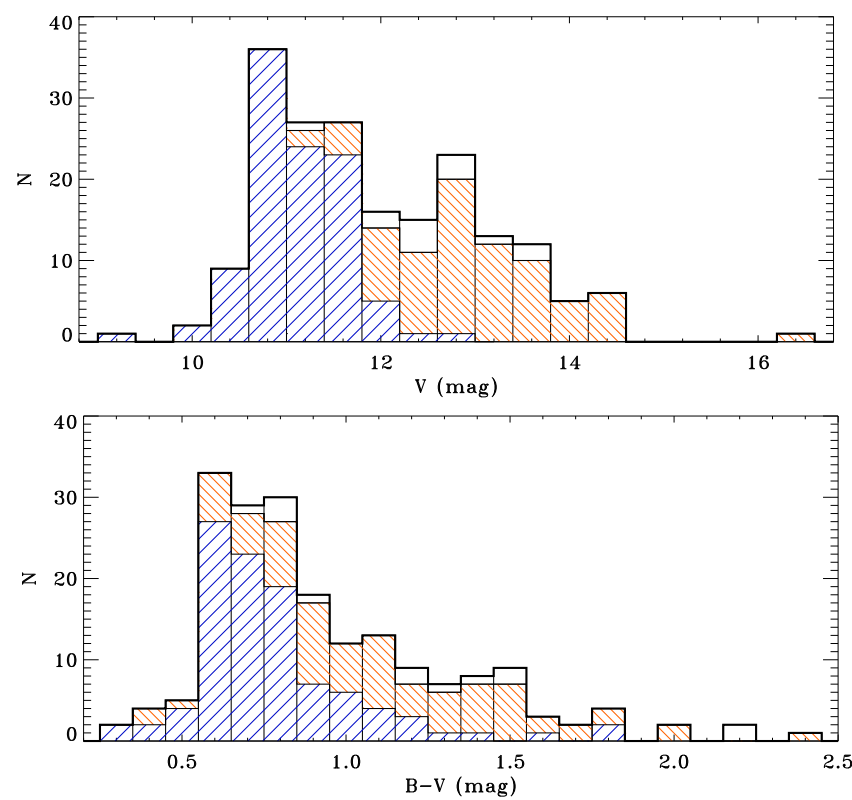

Fig. 2. Distributions of $V$ magnitude (upper panel) and $B-V$ color index (lower panel) for our targets. The blue and red stripes correspond to the Tycho and USNO-B1.0 magnitudes converted into the Johnson $V$ magnitudes based on the relations of Høg et al. (2000) and Lépine \& Shara (2005), respectively (see text for details). The white areas mark the $V$ photographic (GSC II or YB6) ones.

accurate values of $B$ and $V$ retrieved from the AAVSO Photometric All Sky Survey (APASS, Henden et al. 2015) catalog. The $V$ magnitudes are also available in the TASS Mark IV patches photometric catalog (Droege et al. 2006). For most sources we retrieved the $I_{C}$ magnitudes from the TASS catalog or, if not available, from the catalog of Gorbikov \& Brosch (2014). In this way, the dataset of the magnitudes is homogeneous and avoids a mixture of photometric and photographic estimates. We found 177 (plus three whose $V$ magnitude is not provided) and 166 sources in common with the APASS and TASS catalogs, respectively. The different datasets are consistent with the $V$ magnitudes in Table 1, with a Pearson's correlation coefficient (PCC) of $\sim 0.967$. We also note that the $V$ magnitudes in APASS and TASS catalogs are fully consistent with each other (PCC 0.992). In the case of the bright sources $(V \leq 13 \mathrm{mag})$, we obtained a good agreement between the various magnitudes and the spread increases towards the faintest objects, as expected in such a comparison. Regarding the faint objects $(V>13 \mathrm{mag})$, the $V$ magnitudes in Table 1 tend to be overestimated by $0.5 \mathrm{mag}$. This corresponds to the typical accuracy of the Lépine \& Shara (2005) relation at this brightness, which might be subject to systematic errors and other effects.

The photometric standard errors of the Tycho sources with $9<V_{T}<12$ are typically 0.15 and $0.1 \mathrm{mag}$ in $B$ and $V$ passbands, respectively, while they are $\sim 0.25 \mathrm{mag}$ in both bands for the faintest sources. The tabulated errors of the GCS II sources are $\sim 0.3$ mag for the $V$ magnitudes and $\sim 0.4$ mag for both the $B$ and $B_{J}$ magnitudes. The error on the optical magnitudes for the sources fainter than $V=12 \mathrm{mag}$ is considered to be at least of $0.5 \mathrm{mag}$. For our targets, the typical error for the APASS photometry is instead $0.05 \mathrm{mag}$.

Five of the six sources with a $B-V$ color index below our threshold of $0.5 \mathrm{mag}$ come from the inclusion of the Tachihara et al. (2005) sample. The use of APASS magnitudes leads to the same statement for sources \#176 and \#178 only.
Table 2. Instrumental setup summary.

\begin{tabular}{llccr}
\hline \hline Telescope & Inst. & Spectra & Spectral region & $\lambda / \Delta \lambda$ \\
\hline INT $2.5 \mathrm{~m}$ & IDS & 1D & $5800-7200 \AA$ & 9200 \\
CAHA 2.2 m & FOCES & Échelle & $3800-10000 \AA$ & 28000 \\
OHP $1.93 \mathrm{~m}$ & SOPHIE & Échelle & $3872-6943 \AA$ & 39000 \\
\hline
\end{tabular}

Regarding the remaining source (\#102), its color index of $0.546 \pm$ $0.276 \mathrm{mag}$ in the APASS catalog fulfills our initial selection criterion.

\subsection{Observations and data reduction}

To study the physical and kinematic properties of all these young star candidates, we conducted observing campaigns using three spectrographs (Table 2). We acquired both intermediate- and high-resolution optical spectra, sharing our candidates in an optimized way, depending on their brightness.

We observed the brightest ( $V \leq 12 \mathrm{mag}$ ) targets with two instrumental setups. From 26 to 27 September 2009, we used the cross-dispersed échelle SOPHIE ${ }^{1}$ spectrograph (Perruchot et al. 2008; Bouchy et al. 2009) mounted on the $1.93 \mathrm{~m}$ telescope of the Observatoire de Haute Provence (OHP, France). The EEV-4482 detector is a thinned, back-illuminated, anti-reflection coated $4 k \times 2 k 15 \mu \mathrm{m}$-pixel CCD cooled at $-100^{\circ} \mathrm{C}$, with fastreadout mode. We chose the high-efficiency mode equipped with a double-fiber scrambler to homogenize and stabilize the illumination of the spectrograph entrance. This allowed us to record the 39 spectral orders covering effectively the wavelength range $3872-6943 \AA$, with a resolution of about 39000 at $5500 \AA$. The data were treated using the automatic data-reduction pipeline, adapted from the HARPS software ${ }^{2}$.

From 2 to 5 October 2009 and on 12 December 2009, we completed our survey of the bright targets. The échelle spectra were taken with the fibre optics Cassegrain échelle spectrograph (FOCES, Pfeiffer et al. 1998) at the $2.2 \mathrm{~m}$ telescope of the German-Spanish Calar Alto Observatory (CAHA, Sierra de Los Filabres, Spain). We adopted the unique-fiber mode and chose the Site\#1d detector, a $2048 \times 204824 \mu$ m-pixel CCD. We used the configuration with the $400 \mu \mathrm{m}$-slit width, leading to a resolution of about 28000 and covering the wavelength range from 3800 to $10000 \AA$, in a total of 100 orders. Using tasks of the ECHELLE package within the IRAF environment, we reduced the data following the standard steps of background subtraction, division by a flat-field spectrum given by a halogen lamp, wavelength calibration using the emission lines of the Th-Ar arc lamp, and normalization to the continuum through a polynomial fit.

The optically faint candidates $(12<V<14.5 \mathrm{mag})$ were observed with the Intermediate Dispersion Spectrograph (IDS) ${ }^{3}$ at the $2.5 \mathrm{~m}$ Isaac Newton Telescope (INT) of the Observatorio del Roque de los Muchachos (La Palma, Canary Islands, Spain). We acquired long-slit spectra during several observing runs that took place in 4-10 September 2009, 23-28 November 2010, 24-27 October 2012, 27 March to 2 April 2013, 16-20 November 2013, and 18-20 February 2014. During the observing night of 12 to 13 December 2009, some of our targets were

\footnotetext{
1 http://www . obs-hp.fr/guide/sophie/sophie-eng.shtml

2 http://WwW.eso.org/sci/facilities/lasilla/

instruments/harps/

3 http://www.ing.iac.es/Astronomy/instruments/ids/
} 
also observed in queue mode. We used a slit width of 0 '.95 and the $\mathrm{H} 1800 \mathrm{~V}$ dispersion grating with the $235 \mathrm{~mm}$ Camera $(2148 \times 4200$ EEV10a CCD detector until 2010 and $2 k \times 4 k$ RED +2 CCD detector since 2012) and with slow-readout mode. We chose the central wavelength of $6500 \AA$. As the outer regions of the dispersed light beam are severely vignetted by the camera optics, only 2070 of the pixels (roughly from pixel number 1000 to 3070 in the direction of the dispersion) are clear and unvignetted regarding the $\mathrm{H} 1800 \mathrm{~V}$ grating. This allows for efficient coverage of the wavelength range 6200-6800 $\AA$, with a resolving power of about 9200 . For most of our targets and standard stars, we set the slit position angle to the current parallactic angle. Using the typical IRAF tasks, all spectra were debiased, flat-fielded, distortion-corrected, wavelength calibrated (using the emission lines of the $\mathrm{Cu}-\mathrm{Ar}$ and $\mathrm{Cu}-\mathrm{Ne}$ lamps), and finally normalized to the continuum. When a source was already reported as a visual binary in the literature or was identified as such during our observing runs (i.e., with a small angular separation), we aligned the slit on its position angle to simultaneously acquire the spectra of both sources. In this way we made sure to observe the optical counterpart(s) of the unresolved X-ray source. We subsequently examined whether or not the two stars form a physical binary system. When their seeing profiles are partially overlapped on the CCD, we extracted the two spectra by means of a IDL code similar to that of Frasca et al. (1997), rather than the IRAF task APALL (see Appendix A for details). The use of a double Gaussian profile optimizes the spectrum extraction of the fainter source, which may be heavily contaminated by the brighter one.

We removed the telluric water vapor lines only in the spectral region around the $\mathrm{H} \alpha$ line of every spectrum, in all instrumental setups. We applied a method similar to that described by Frasca et al. (2000) using telluric templates taken with the same instrumentation as the targets. We show a few results for the case of SOPHIE spectra (Fig. E.1) to illustrate its effectiveness.

Owing to bad weather conditions during our IDS observing runs, we were unable to obtain a spectrum for 35 of our targets. All the unobserved targets have a lower priority because their Galactic latitude is higher than $25^{\circ}$ or they were not classified as WTTSs by Tachihara et al. (2005). Figure E.2 shows the spectra acquired in the region around the $\mathrm{Li}$ I $\lambda 6707.8$ lines.

\section{Analysis and results}

\subsection{Radial velocity and multiplicity}

We measured the heliocentric radial velocities $(R V \mathrm{~s})$ using the cross-correlation technique. We distinguished spectroscopic systems from single stars (or single-lined binaries) based on the number of peaks visible in the cross-correlation function (CCF).

Regarding the IDS data, we cross-correlated each long-slit spectrum of our targets with that of RV-standard stars observed during the same night. For this purpose, we have upgraded the IDL procedure described in Klutsch (2008) and Paper I. We masked the spectral range around the $\mathrm{H} \alpha$ line because of its strong wings and the possible chromospheric emission in the core that can considerably broaden and distort the $\mathrm{CCF}$ peak. For the same reason we discarded the spectral regions strongly affected by telluric absorption lines. When only one CCF peak was detected, the code performs an additional analysis to report any asymmetry of the CCF profile, possibly interpreted as an SB2 system observed near the conjunction. Such a procedure is only relevant when the masses of the two stars differ significantly. We computed the errors of the pixel shift from the center of the Gaussian function fitting the CCF. We computed the $R V$ errors as a function of the fitted peak height and the antisymmetric noise through the $R$ factor, as described in Tonry \& Davis (1979).

For the FOCES data we derived the $R V$ values with the IRAF task FXCOR, by cross-correlating each échelle order of the target spectra with the corresponding one of the most similar RV-standard star, which was acquired during the same run. We discarded the orders heavily contaminated by telluric lines or those including very broad lines and activity indicators, such as the Ca II $\mathrm{H} \& \mathrm{~K}$ lines, the Balmer series $(\mathrm{H} \alpha$ and $\mathrm{H} \beta)$, and the $\mathrm{Ca}$ II IR triplet lines. We fitted the entire CCF peak with a Gaussian to measure more precisely the $R V$ values, which were obtained as the weighted average of all the individual $R V$ measurements computed by FXCOR per each échelle order. The resulting $R V$ uncertainty is the standard error of the weighted mean, $\sigma_{\mathrm{RV}}$.

The $R V$ measurements derived from the target spectra acquired with the SOPHIE spectrograph were performed with the cross-correlation analysis that is included in the automatic data-reduction pipeline developed by the OHP staff. We used the most appropriate mask depending on the $B-V$ color index (or spectral type) of the target, among the five available ones. This procedure failed for some of the multiple systems and fast rotators. From a first guess of their spectral type (Sect. 3.2), we selected the most appropriate synthetic spectrum from the POLLUX database (Palacios et al. 2010) to be used as template for computing the $\mathrm{CCF}$, which was automatically fitted by a procedure similar to that used for IDS data. Taking advantage of the high resolution of these spectra, we improved the code to detect and fit the CCF peak of a companion even having a much lower brightness in a stellar binary system, that is, with a low mass-ratio. This requires careful analysis of the $\mathrm{CCF}$ residues after subtracting the best fit, especially when the two peaks are severely blended (i.e., one CCF peak with an asymmetric shape).

Table 3 contains all our $R V$ measurements and associated errors, with one entry for each acquired spectrum and, in case of multiple systems, for each component.

Since our IDL procedure and the task FXCOR use the RVstandard stars as templates, we need an accurate $R V$ value for them. So we used the $R V$ measurements available from the Élodie archive ${ }^{4}$, which were derived from high-resolution spectra. Table E.1 lists all these values that agree with those in Nidever et al. (2002) or other compilations of $R V$ measurements (e.g., Gontcharov 2006; Kharchenko et al. 2007).

For multiple systems, we adopted an independent Gaussian fit for each CCF peak whenever they are not blended (i.e., far from the conjunctions). We used instead a multiple-Gaussian fit algorithm when the spectral lines are partially blended. Moreover, we automatically looked for any significant peaks in the $\mathrm{CCF}$ and fitted them with single, double, or multiple functions (i.e., up to four Gaussian profiles), using our IDL code that computes the CCF and treats the errors as FXCOR does. We refer the reader to Paper I for details on this code and its performances.

Among the 47 sources observed at multiple epochs, eleven sources display a large variation in radial velocity (Table 3 ). We classified those with $R V$ change significantly larger than the $R V$ errors as single-lined binaries (SB1) and those with smaller $R V$ variation as possible SB1 systems (SB1?). We discovered that 44 of the surveyed sources are multiple spectroscopic systems, which are distributed as follow: nine SB1 (4.8\%), 28 doublelined (SB2) plus four likely SB2 (17.2\%), and three triple (SB3; $1.6 \%$ ) systems (see Table B.2). This corresponds to $20 \%$ of the

4 http://atlas.obs-hp. fr/elodie/ 


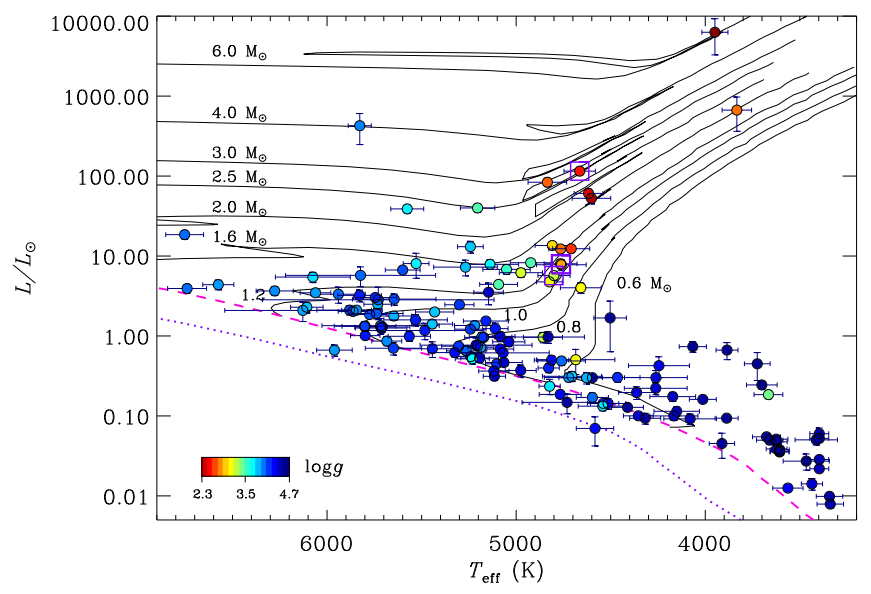

Fig. 3. HR diagram of targets with known APs and parallaxes. The symbols are color coded by $\log g$ derived from spectra (Sect. 3.2). The four lithium-rich sub-giant or giant stars are enclosed in open squares (Sect. 3.4). The evolutionary tracks from the ZAMS of Girardi et al (2000) are shown as solid lines with the labels representing their masses. The dashed and dotted lines display the ZAMS with solar metallicity ( $Z=0.019)$ and $Z=0.001$ by the same authors, respectively.

overall sample (i.e., our targets, plus the 20 sources listed in Table B.1 and the eight sources from Papers II and III) and to $24 \%$ of the sources with new observations presented here, as listed in Table 3. For the 17 sources classified as possible spectroscopic systems (Table B.2), additional observations are needed to draw firm conclusions on their multiplicity.

\subsection{Spectral classification and projected rotational velocity}

We applied the IDL code ROTFIT (Frasca et al. 2006) to perform the physical characterization of SB1 systems and single stars, that is, those with only one peak in the CCF profile. This code searches for the best combination of parameters by comparing the target spectrum with reference star spectra that are rotationally broadened until a minimum $\chi^{2}$ was reached. As in our previous works (Papers I and III), we determined the spectral type (SpT), effective temperature $\left(T_{\text {eff }}\right)$, gravity $(\log g)$, and metallicity $([\mathrm{Fe} / \mathrm{H}])$ using as templates a library of 270 high-resolution spectra of low-activity and slowly rotating stars retrieved from the Élodie archive (Moultaka et al. 2004). For these stars we took the atmospheric parameters (APs) from the PASTEL catalog (Soubiran et al. 2010). We report these results in Table 3 in which any source observed at several epochs ${ }^{5}$ has multiple entries. The typical accuracy on the spectral type is about one subclass. We refer the reader to Appendix $\mathrm{C}$ for a description of our procedures dedicated to the analysis of the M-type stars.

To minimize possible effects on projected rotational velocities $(v \sin i)$ due to different resolutions and instrumental setups between the target spectra (acquired with both IDS and FOCES) and the Élodie ones we also used a smaller library of template spectra taken with these spectrographs during our observing runs. Table E.1 lists them as well as all their relevant information. Analogous to the treatment of the full library of Élodie templates, the rotational velocity of each template is progressively increased by convolving its spectrum with a rotational profile of a given $v \sin i$. The best match of the template spectrum with the target spectrum, found by $\chi^{2}$ minimization, gives

\footnotetext{
5 Whether with different instruments (usually for the bright sources) or with the same instrumental setting (in case of the faint ones).
}

us the value of $v \sin i$. We note that most $v \sin i$ values derived with the Élodie templates are slightly underestimated compared to those obtained when the spectra are taken with the same instrumental setup. This is probably caused by the resampling of the Élodie spectra on the points of the IDS or FOCES ones. We thus adopted the $v \sin i$ values obtained with the template acquired with the same spectrograph as the target (Table 3). At the IDS, FOCES, and SOPHIE resolutions, any $v \sin i$ determination is reliable only if the $v \sin i$ value is larger than $15 \mathrm{~km} \mathrm{~s}^{-1}$, $5 \mathrm{~km} \mathrm{~s}^{-1}$, and $2 \mathrm{~km} \mathrm{~s}^{-1}$, respectively. We determined these lower limits with simulations similar to those made by Frasca et al. (2015).

\subsection{Stellar luminosity and extinction}

We analyzed the spectral energy distribution (SED) of our targets with the aim of checking the spectroscopic atmospheric parameters, of estimating the extinction, $A_{V}$, and of deriving the stellar luminosities. The $B V I_{C} J H K_{s}$ SEDs were constructed using the optical and NIR photometric data described in Sect. 2.2. We completed them with mid-infrared photometry provided by the WISE All-Sky survey (Wright et al. 2010; Cutri et al. 2012).

Figure 3 shows the Hertzsprung-Russell (HR) diagram for the targets with accurate parallaxes (Table 4) and APs derived in the present study (Table 3). For 187 of our targets we made use of the Gaia DR2 parallax, while we adopted the values of $18.4 \pm 2.7$ mas and $36.0 \pm 6.9$ mas for the sources \#15 and \#101, respectively, as quoted in the URAT Parallax Catalog (Zacharias et al. 2015; Finch \& Zacharias 2016). No parallax is available for the remaining four targets. Evidently, the values of $\log g$ found by our spectroscopic analysis are fully consistent with the evolutionary status in the HR diagram, demonstrating the reliability of APs derived by ROTFIT (Sect. 3.2). Most targets are located close to (or slightly above) the zero-age main sequence (ZAMS) and show $\log g>3.5$. These are good young star candidates. As for the 16 sub-giant or giant stars, the X-ray emission can be related to their evolutionary status or to the presence of an unseen companion, which forces the evolved star into fast rotation.

We adopted the grid of NextGen low-resolution synthetic spectra, with $\log g$ in the range 3.5-5.0 and solar metallicity by Hauschildt et al. (1999), to fit the optical-NIR portion (from $B$ to $H$ band) of the SEDs, in the same way as in Paper III. We fixed $T_{\text {eff }}$ and $\log g$ of each target to the values found with ROTFIT (Table 3 ) and let the stellar angular diameter and the extinction $A_{V}$ vary until a minimum $\chi^{2}$ was reached. For the stars with a known distance, this also provides us with a measure of the stellar radius and luminosity that was obtained by integrating the best-fit model spectrum. As listed in Table 4, we found low extinction values $\left(A_{V}<0.5 \mathrm{mag}\right)$, with the exception of the more distant targets, which are mostly sub-giant or giant stars.

In line with our selection criteria, most of our targets are consistent with late-type stars near the main sequence (Fig. 3), with no or low reddening (Table 4). These results fully agree with the distribution of our targets in the infrared color-color diagram (Fig. 4). We found nine sources that lie outside the locus of mainsequence (MS) stars. For a better readability the source \#120 with $H-K_{s}=-0.152 \pm 0.045 \mathrm{mag}$ is not shown. Sources \#8 and \#160 likely suffer from extinction, as they lie at the upper right corner in the color-color diagram $(J-H>0.9 \mathrm{mag}$ and $\left.H-K_{s}>0.3 \mathrm{mag}\right)$. These color excesses $E(J-H)$ and $E\left(H-K_{S}\right)$ allow us to estimate their extinction $A_{V}$ by means of the midinfrared extinction law of Wang \& Chen (2019) and the standard optical one $R_{V}=3.1$. We classified the source \#8 as a 


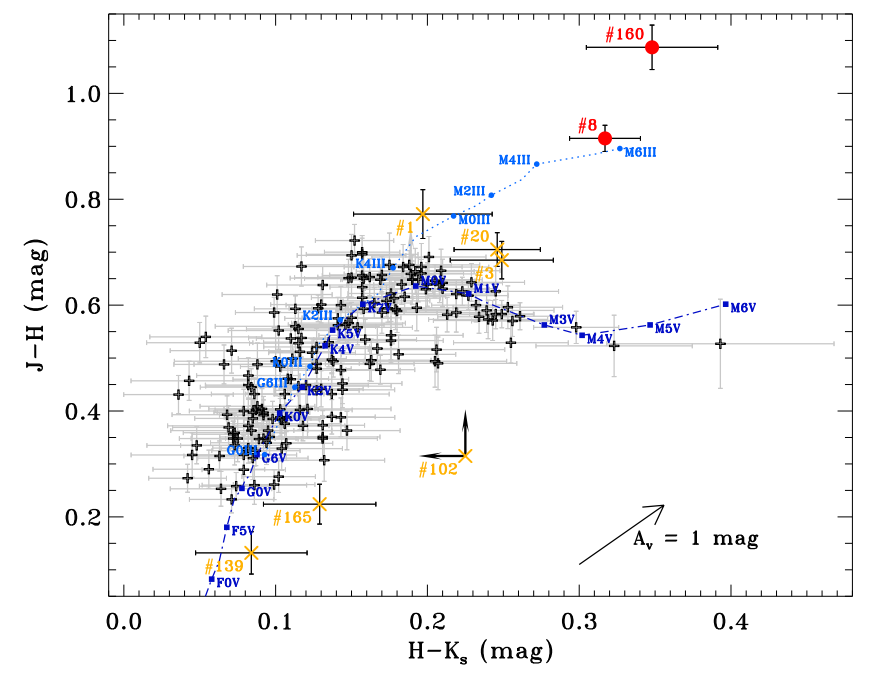

Fig. 4. $\left(H-K_{s}, J-H\right)$ color-color diagram of our targets. We also display the intrinsic color tracks of main-sequence (MS; dash-dotted line) and giant (dotted line) stars from Bessell \& Brett (1988), which we converted into the 2MASS photometric system using the Carpenter (2001) relation. Eight sources lie outside the locus of MS stars: two sources likely suffering from extinction (red circles) and six sources with possibly small extinction or poor-quality infrared magnitudes (orange crosses). We also overplot their error bars, except for source $\# 102$ because its $J$ and $K_{s}$ magnitudes are upper limits in 2MASS. The arrow indicates the reddening vector for $A_{V}=1 \mathrm{mag}$. For a better readability, the source \#120 with $H-K_{s}=-0.152 \pm 0.045$ mag does not appear.

K5 giant star with a temperature $T_{\text {eff }}=3833 \pm 78 \mathrm{~K}$ and a surface gravity $\log g=2.84 \pm 0.44$, while its infrared colors place it near the position of an M6 giant star, implying an extinction of $\sim 2$ mag. Moreover, this source is at least 2 mag fainter than giants having a similar $J-K_{s}$ color index in the LSPM-North catalog (Lépine \& Shara 2005). This is consistent with a high reddening, as also indicated by the SED analysis. Source \#160 is a known member of the L1251 cloud (Kun \& Prusti 1993) and classified as a WTTS with spectral type K5 (Kun et al. 2009), implying an extinction of $\sim 3 \mathrm{mag}$. The other sources outside the locus of MS stars include two visual binary candidates (\#102 and \#120, see Table 5), one spectroscopic system (\#20), and four possible single stars (\#1, \#3,\#139, and \#165). We note that the entire photometry of the source \#139 could be affected by the extended halo of light of the nearby B5 binary V447 Cep (see Appendix D).

\subsection{Lithium content and age}

Lithium is a fragile element that is progressively depleted in the deep convective envelopes of late-type stars where it is brought at temperatures of about $3 \times 10^{6} \mathrm{~K}$. Therefore, the strength of the Li I $\lambda 6707.8 \AA$ line can be used as an age proxy for dwarfs with spectral type later than mid-G; for these stars, a high lithium abundance is a clear sign of youth (e.g., Soderblom et al. 1998).

In all instrumental setups used in this study, the lithium line and the nearby Fe I $\lambda 6707.4 \AA$ line are blended, at least partly. However, when applying the spectral subtraction technique (see, e.g., Herbig 1985; Barden 1985; Frasca \& Catalano 1994; Montes et al. 1995), this contamination is automatically corrected because the best-fitted lithium-poor template, which is rotationally broadened by ROTFIT to the $v \sin i$ of the target, faithfully reproduces the spectral behavior around the lithium line (Fig. E.2). We measured the Li I equivalent width, $W_{\mathrm{Li}}$, for the whole sample of single stars and SB1 systems (Table 3). We then derived the lithium abundance, $\log \mathrm{N}(\mathrm{Li})$, based on the Pavlenko \& Magazzu (1996) calculations.

Following the classification of Papers I and III, we defined as Pleiades-like the stars lying between the lower and upper envelopes of the Pleiades cluster in the $T_{\mathrm{eff}}-W_{\mathrm{Li}}$ diagram (filled squares in Fig. 5). Since the lower envelope of the Pleiades nearly coincides with the upper envelope of the $300 \mathrm{Myr}$-old UMa cluster (Soderblom et al. 1993a), the age of Pleiades-like objects should range between 100 and 300 Myr. Analogously, we have considered as PMS-like the stars lying above the Pleiades upper envelope (filled circles in Fig. 5).

In the literature, a significant scattering of $\log \mathrm{N}(\mathrm{Li})$ was found for stars belonging to the same cluster. This can be due to a real age spread within the cluster or to further parameters affecting the lithium depletion, such as stellar rotation (e.g., Jeffries 2017; Bouvier et al. 2018, and references therein). For a field star, this prevents any direct conversion of $\log \mathrm{N}(\mathrm{Li})$ into age.

However, a relative age estimate can be obtained by comparing the position in the $T_{\text {eff }}-\log \mathrm{N}(\mathrm{Li})$ diagram with that of stars with a well-defined age and similar APs. We therefore overplot the envelopes of clusters IC 2602, Pleiades, and Hyades (see, e.g., Soderblom et al. 1993b,a; Jeffries et al. 2000), along with the locus of known members of the $\beta$ Pictoris and TucanaHorologium associations whose quoted ages are about 12 and $30 \mathrm{Myr}$, respectively (da Silva et al. 2009). As a result, most PMS-like stars are distributed similarly to the $\beta$ Pictoris members and slightly above those of the Tucana-Horologium association (Fig. 5).

We got an alternative estimate of the ages of our targets by comparing their position in the HR diagram with pre-mainsequence isochrones from Siess et al. (2000), as shown in Fig. 6. Most PMS-like stars are located between the isochrones at 10 and 30 Myr, while six sources (\#6 c2, \#189, \#191 c1 \& c2, F3, F4) are found between the isochrones at 1 and $10 \mathrm{Myr}$ (Fig. 6). This agrees with the young age assumed in Paper II. In contrast, the Pleiades-like stars are mostly below the isochrone at $30 \mathrm{Myr}$. We are finally able to confirm that all the PMS-like and Pleiades-like sources are likely young stars, with the exception of four sources (\#109, \#133, \#159, \#185 c1), which are likely lithium-rich sub-giant or giant stars (Figs. 3 and 6).

\subsection{Calculation of spatial-kinematic coordinates}

To study the kinematics of our targets in the $X Y Z U V W$ space, we combined the average radial velocities derived in Sect. 3.1 with the sky positions, proper motions, and parallaxes reported in Gaia DR2. For sources \#15 and \#101, we actually derived their kinematics by making use of the sky positions from the 2MASS catalog and the astrometry from the URAT Parallax Catalog. We then computed the Galactic positions $(X, Y, Z)$ and heliocentric space-velocity components $(U, V, W)$ in the left-handed coordinate system 6 . All these values are listed in Table 4.

As shown in Fig. 7, the $(U, V)$ plane of targets with astrometry and $R V$ measurement (Table 4 ) shows that most PMS-like and Pleiades-like stars are located in the young-disk (YD) population in the solar neighborhood, according to Eggen (1996). However we found three PMS-like (\#35, \#146, \#185c1) and two Pleiades-like (\#46, \#109) in the region populated by olddisk stars. While the sources \#109 and \#185c1 are Li-rich giant

\footnotetext{
${ }^{6} X$ and $U$ are pointing towards the Galactic anti-center; $Y$ and $V$ in the
} Galactic rotation direction; $Z$ and $W$ towards the North Galactic pole. 

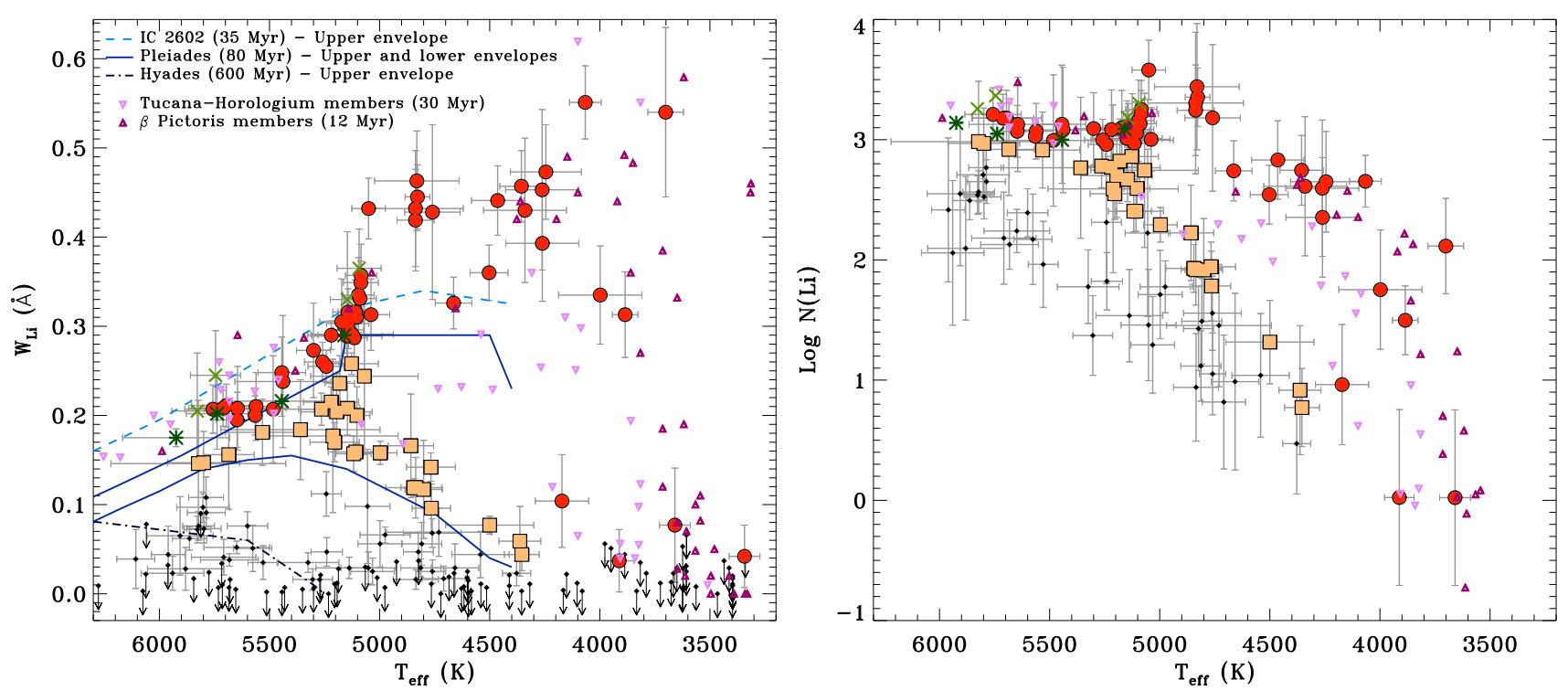

Fig. 5. Left panel: Li I $\lambda 6707.8$ equivalent widths as a function of effective temperatures for our targets. Since each symbol results from the analysis of a unique spectrum (Table 3), any source observed at multiple epochs is marked several times. The arrow symbols indicate the upper limits on lithium equivalent width. The lines of different color and style mark the boundaries for three young clusters. Right panel: lithium abundances versus effective temperatures. We disregard the upper limits. In both panels, we display the PMS-like (big filled circles), Pleiades-like (filled squares), and older (filled diamonds) stars (see Sect. 3.4 for details). The four comoving TTSs towards the CO Cepheus void (Paper II) and the additional members (Paper III) are highlighted with asterisks and crosses, respectively. For comparison, we also overplot the locus of the sources belonging to two young (age $=12-30 \mathrm{Myr}$ ) associations listed by da Silva et al. (2009) with different small triangles.

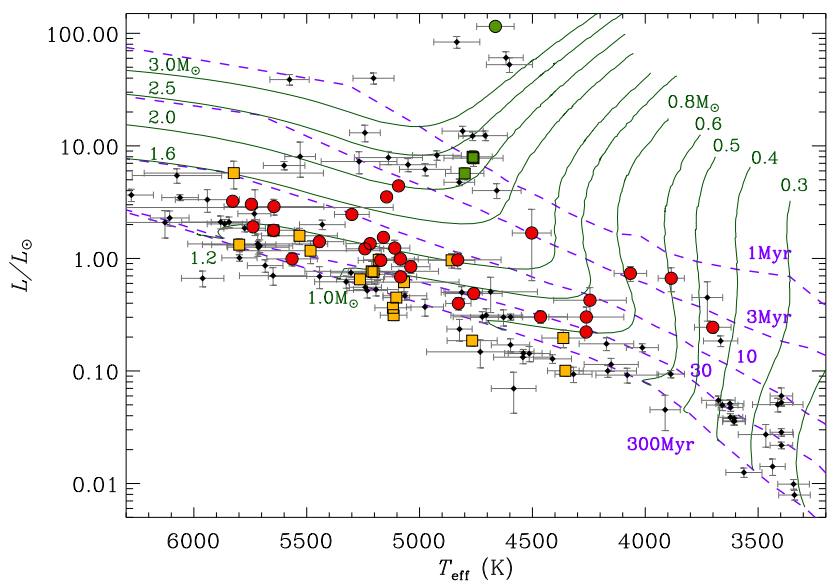

Fig. 6. HR diagram of our targets with known APs and parallaxes. We display the young stars selected as PMS-like (red circles) and Pleiadeslike (orange squares) sources, along with the lithium-rich sub-giant or giant stars (green symbols) and older (black diamonds) sources. The pre-main-sequence evolutionary tracks of Siess et al. (2000) are shown as solid lines with the labels representing their masses. The dashed lines denote the isochrones at ages of 1, 3, 10, 30, and $300 \mathrm{Myr}$.

stars, additional observations are needed to confirm any possible variation in radial velocities for the others. Our observations reveal that the sources \#35, \#46, \#109 are possible SB2 systems due to a double-peaked $\mathrm{H} \alpha$ profile or their CCF shape (i.e., slightly asymmetric or with a possible small peak below our detection limit; Table 3). Regarding source \#179, we do not exclude a problem in the wavelength calibration. From our $R V$ value of $-147.8 \pm 0.8 \mathrm{~km} \mathrm{~s}^{-1}$, this source might be located in the old-disk (OD) area. Thus this $R V$ value must be used with care, especially because this source is not a high-proper-motion star and its $R V$ value in Gaia DR2 is of $-9.74 \pm 2.88 \mathrm{~km} \mathrm{~s}^{-1}$, which we use afterwards (Table 4). Finally, we note that the signal-

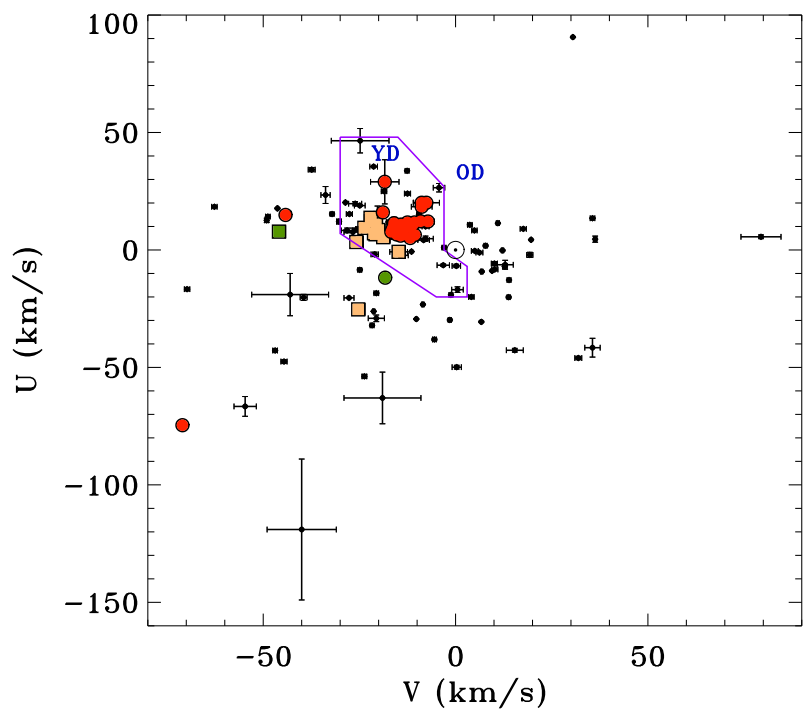

Fig. 7. $(U, V)$ plane of targets with astrometry and $R V$ value (Table 4). The solid line marks the separation between the young- (YD; age $<2$ Gyr) and old-disk (OD) populations in the solar neighborhood, according to Eggen (1996). We use the same symbols as in Fig. 6.

to-noise of the spectrum acquired for source \#146 is not high enough to obtain an accurate $W_{\mathrm{Li}}$ value.

Figure 8 shows the distribution of our targets in various $2 \mathrm{D}$ planes of the $X Y Z U V W$ space restricting the parameter ranges to the locus of the young sources. We used the probabilistic approach described in Klutsch et al. (2014) to assess their membership to the five young stellar kinematic groups denoted in Fig. 8. Their ages are adopted from Table 1 of Montes et al. (2001). In the kinematic velocity space, the populations of PMSlike and Pleiades-like stars are located in well-distinguishable regions. On the one hand, the heliocentric space-velocity 

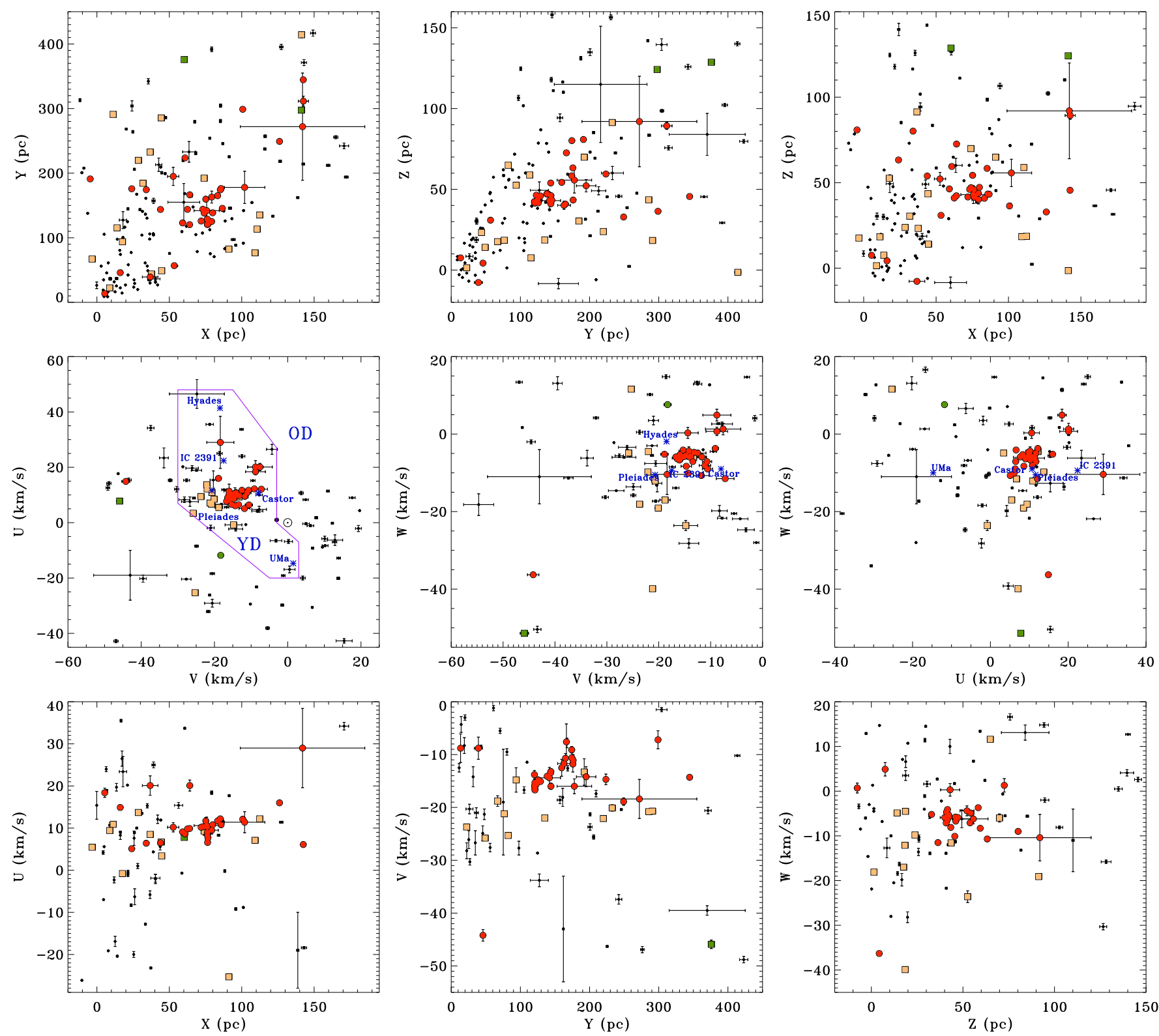

Fig. 8. Distribution of our targets in Galactic coordinates (upper panels) and space-velocity components (middle panels), along with the diagram of position versus velocity pointing in a given direction (lower panels). We restricted the parameter ranges on all diagrams to better see the distribution of the youngest sources. We use the same symbols as in Fig. 6. Middle panels: we also display the mean position of the IC 2391 ( $\sim 50 \mathrm{Myr})$ and Hyades ( $\sim 600 \mathrm{Myr})$ superclusters, Pleiades ( $\sim 100 \mathrm{Myr})$ and Castor ( $200 \mathrm{Myr})$ moving groups, and Ursa Major (UMa) group ( 300 Myr). In the $(U, V)$ plane, the solid line delimits the area of young- and old-disk populations in the solar neighborhood (Eggen 1996).

components of a compact group of PMS-like stars are marginally consistent with those of the Castor moving group. Nevertheless, such a link is hardly compatible because of the age difference between these two groups of young stars, but above all because the PMS-like stars are only weakly dispersed in the XYZUVW space. On the other hand, $75 \%$ of the 12 Pleiades-like single stars with an $R V$ value in Table 4 have membership probability to the Pleiades moving group larger than $40 \%$. These nine sources are $\# 16, \# 22$, \#102, \#112, \#123, \#125, \#129, \#151, and \#179. Our procedure disregarded two sources $(\# 31 \mathrm{c} 2, \# 86)$ in the YD area due to a space-velocity component $W$ lower than $-23 \mathrm{~km} \mathrm{~s}^{-1}$, along with source \#46 located in the OD area (Fig. 7). These three sources probably exhibit some variation in radial velocity.

\section{Discussion}

\subsection{Properties of the sample}

Our study confirms that the multivariate analysis allows for the optimization of the disentangling of the stellar population from X-ray emitting extragalactic components. In fact, only one (\#99) of the 162 sources is wrongly classified as a star due to a rather singular spatial configuration of the different sources (Fig. D.3) and the photometric properties of this galaxy that are similar to late M-type stars (Appendix D). The main stellar populations in our sample are young objects, multiple systems (Appendix B), and low-mass stars (Appendix C). This latter group includes 36 M-type dwarfs (i.e., from M0 to M4.5; see Table C.1). 


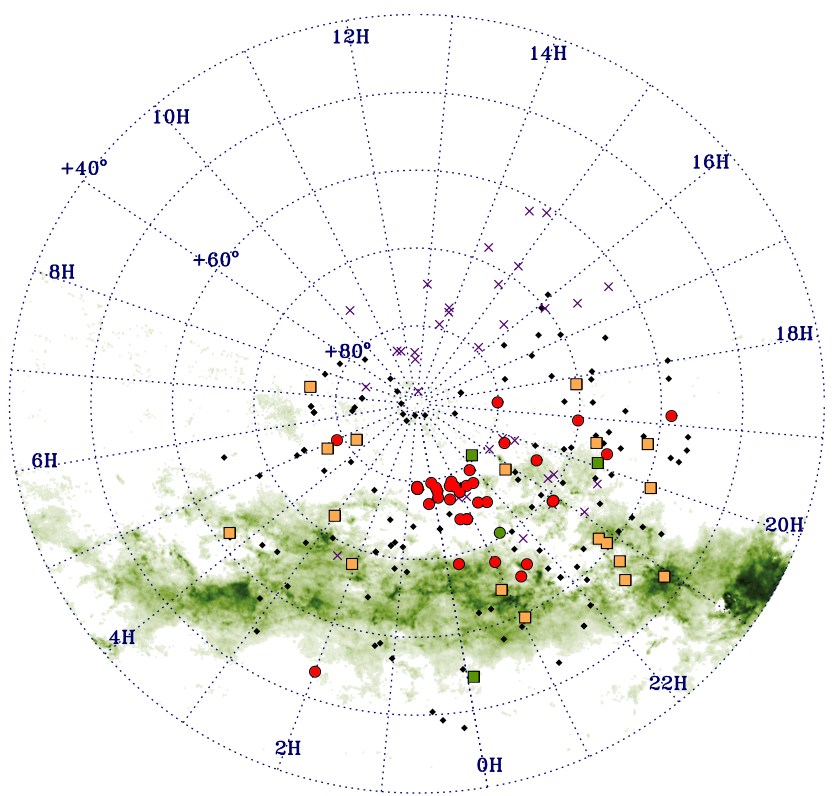

Fig. 9. Spatial distribution of our targets, overplotted on the extinction $\left(A_{V}\right)$ map of Dobashi et al. (2005). In addition to the symbols defined in Fig. 6, we denote the unobserved sources with crosses.

We selected young stars more efficiently than in our previous works. This may be linked to a sampling of more distant sources in comparison to previous studies, as explained by the Gould Disk scenario (Guillout et al. 1998b). It could also be related to the sky region considered here in which stars younger than $30 \mathrm{Myr}$ are found at three different distance scales: the visual binary V368 Cep and its comoving companion NLTT 56725 ( 20 pc; Makarov et al. 2007), the Cepheus association (Table 6), and the Cepheus Flare region (Kun 2008; Kun et al. 2008).

We identified 59 lithium-rich sources. Most of them prove to be young but four sub-giant or giant stars also display a strong lithium line in absorption (Sect. 3.4). This is consistent with the discovery of several lithium-rich giants from our previous studies of the stellar X-ray population (Papers I and III). We classified 18 targets as Pleiades-like sources (age $=100-300 \mathrm{Myr}$ ) and 37 as PMS stars (age $=10-30 \mathrm{Myr}$ ). These two distinct stellar populations are found in different sky areas (Fig. 9). While the former are mainly located towards the Galactic plane, the latter are mostly projected in front of the $\mathrm{CO}$ Cepheus void, in the Cepheus Flare region.

During our survey of 186 sources, we identified 44 (24\%) spectroscopic multiple systems. This fraction is $30 \%$ lower with respect to Papers I and III. Nine and six of them also display a strong and very strong lithium line (Table B.2), respectively. Using Gaia DR2 data, we found that 40 of our targets and six of eight young stars in Papers II and III have comoving companions with an angular separation ranging from a few arcsecond to a few arcmin (Table 5). Five of these visual binaries (\#6, \#18, \#119, \#131, G4) also have one component classified as an SB2 system. They are, therefore, likely hierarchical triple systems.

\subsection{Young stars towards the CO Cepheus void}

The possible detection of a new young association in Cepheus (age $=10-20 \mathrm{Myr}$; Paper II) is supported by our identification of 20 PMS stars (plus three small-separation companions observed during our spectroscopic campaigns) in the same region (Fig. 10) and our discovery of their eight comoving companions (Table 5). These are overplotted on the visible extinction $A_{V, \mathrm{RQ}}$ map of Planck Collaboration XXIX (2016) ${ }^{7}$, derived from the thermal dust emission modeling presented by Draine \& Li (2007). The PMS stars are highly clumped near the Galactic coordinates $(X, Y, Z)=(76,130,43) \mathrm{pc}($ Fig. 8$)$. To better see their distribution, we zoomed in this smaller region (Fig. 11).

Almost all PMS stars towards the CO Cepheus void are related to the Cepheus association (Fig. 10), except for sources $\# 160$ and \#189 that are located at larger distances $(354 \pm 9$ pc and $320 \pm 97$ pc, respectively). The former is a known member of the molecular cloud L1251 (see Sect. 3.3), while the astrometry of the latter suffers from large uncertainties. According to the $6 \mathrm{D}$ phase-space data of the remaining PMS stars in this restricted area, we can divide them into three subgroups, as explained below. We summarize their properties in Table 6 .

We first identified the highest density of PMS stars in the projection of the sky positions (Fig. 10) and in the various planes in Galactic coordinates (Fig. 11). Up to now, this group (henceforth Cep I) is composed of one visual binary (G3) and two triple hierarchical systems (\#6 and G4). It is highlighted by a hexagon in Figs. 10 and 11. Moreover, source \#6 has an additional wideseparation companion (Appendix B.2).

Five additional young sources with similar properties are located in a more extended area (henceforth Cep II; cyan circles in Figs. 10 and 11). In addition to the three Cep I members, this group includes two single stars (\#158 and \#193), one SB1 system (\#153), and two visual binaries (\#188 and G1).

Finally, the largest group (henceforth Cep III; blue circles in Figs. 10 and 11) is composed of five single stars (\#142, \#182, $\# 186$, F2, F4), one SB1 system (\#156), four visual binaries (\#152, \#191, G2 and F3), and the Cep II members. The source F3 has also a wide-separation companion (Table 5).

\subsection{Additional CO Cepheus void stars from Gaia}

To see the extent of the association in Cepheus, we built a new sample that is hereinafter referred to as Gaia-2MASS. We selected all sources brighter than $K_{s}=12$ mag from the PPMXL catalog (Roeser et al. 2010), using a cone search centered at $(\mathrm{RA}$, Dec $)=\left(0^{\mathrm{h}},+77^{\circ}\right)$ with a radius of $6^{\circ}$, which covers all the sky area devoid of interstellar matter. Subsequently we matched this dataset with Gaia DR2 (radius = 1").

An asymmetry is clearly visible in the distribution of the proper motions in right ascension (middle panels of Fig. 12), in particular in the range of proper motions of the members of the Cepheus association (delimited by vertical lines). This results in an excess of sources with positive $\mu_{\alpha} \cos \delta$ values with respect to a normal symmetric distribution. The total number of the excess Gaia-2MASS sources is significantly larger than the number of young stars identified up to now in the CO Cepheus void. To search for a possible link between this excess of Gaia-2MASS stars and the Cepheus association, we performed a comparison with a simulation based on the Besançon model (Robin et al. 2012; Czekaj et al. 2014; Luri et al. 2014).

Due to the right ascension of the members of the Cepheus association ranging from $22 \mathrm{~h}$ to $1 \mathrm{~h}$, we have to use a rectan-

\footnotetext{
7 The map results from the renormalization of the visible extinction one based on the analysis of quasi-stellar objects observed in the Sloan Digital Sky Survey. This $A_{V, \mathrm{RQ}}$ map is available from the Planck Legacy Archive (http://pla.esac.esa.int/pla/ \#home) and corresponds to the file: COM_CompMap_Dust-DL07AvMaps_2048_R2.00.fits[AV_RQ].
} 
Table 6. Properties of the three groups of PMS stars in Cepheus.

\begin{tabular}{llccc}
\hline \hline Parameter & Unit & Cep I & Cep II & Cep III \\
\hline$\langle l\rangle$ & {$\left[{ }^{\circ}\right]$} & $122.31 \pm 0.04$ & $120.7 \pm 2.0$ & $118.7 \pm 2.8$ \\
$\langle b\rangle$ & {$\left[{ }^{\circ}\right]$} & $16.31 \pm 0.14$ & $16.37 \pm 0.33$ & $15.7 \pm 1.7$ \\
$\left\langle\mu_{\alpha} \cos \delta\right\rangle$ & {$\left[\mathrm{mas} \mathrm{yr}^{-1}\right]$} & $23.88 \pm 0.29$ & $22.63 \pm 0.25$ & $20.35 \pm 0.34$ \\
$\left\langle\mu_{\delta}\right\rangle$ & {$\left[\mathrm{mas} \mathrm{yr}^{-1}\right]$} & $-3.11 \pm 0.25$ & $-0.31 \pm 0.51$ & $1.98 \pm 0.49$ \\
$\langle R V\rangle$ & {$\left[\mathrm{km} \mathrm{s}^{-1}\right]$} & $-9.05 \pm 0.24$ & $-8.98 \pm 0.3$ & $-8.30 \pm 0.43$ \\
$\langle\pi\rangle$ & {$[\mathrm{mas}]$} & $6.713 \pm 0.016$ & $6.439 \pm 0.054$ & $6.044 \pm 0.094$ \\
$\langle$ Trigo. distance $\rangle$ & {$[\mathrm{pc}]$} & $148.97 \pm 0.36$ & $155.7 \pm 1.3$ & $167.7 \pm 2.9$ \\
$\langle X\rangle$ & {$[\mathrm{pc}]$} & $76.43 \pm 0.19$ & $76.0 \pm 0.5$ & $75.9 \pm 1.0$ \\
$\langle Y\rangle$ & {$[\mathrm{pc}]$} & $120.86 \pm 0.29$ & $128.5 \pm 1.4$ & $141.7 \pm 2.7$ \\
$\langle Z\rangle$ & {$[\mathrm{pc}]$} & $41.75 \pm 0.13$ & $43.9 \pm 0.4$ & $45.1 \pm 0.8$ \\
$\left\langle U_{\odot}\right\rangle$ & {$\left[\mathrm{km} \mathrm{s}^{-1}\right]$} & $7.90 \pm 0.29$ & $9.15 \pm 0.28$ & $9.99 \pm 0.33$ \\
$\left\langle V_{\odot}\right\rangle$ & {$\left[\mathrm{km} \mathrm{s}^{-1}\right]$} & $-16.08 \pm 0.26$ & $-15.54 \pm 0.24$ & $-14.02 \pm 0.44$ \\
$\left\langle W_{\odot}\right\rangle$ & {$\left[\mathrm{km} \mathrm{s}^{-1}\right]$} & $-5.43 \pm 0.26$ & $-5.70 \pm 0.15$ & $-5.42 \pm 0.27$ \\
Age & {$[\mathrm{Myr}]$} & $15 \pm 5$ & $15 \pm 5$ & $15 \pm 5$ \\
\hline Number of targets $[$ sources] & $(:)$ & $3[7]$ & $8[14]$ & $18[29]$ \\
Fraction of systems & $100 \%$ & $75 \%$ & $61 \%$ \\
Number of single stars & 0 & 2 & 7 \\
Number of SB1 systems & 0 & 1 & 2 \\
Number of visual binaries & 1 & 3 & 7 \\
Number of multiple systems & 2 & 2 & 2 \\
\hline
\end{tabular}

Notes. ${ }^{(\ddagger)}$ We give for each group the number of targets listed in Table 1, while that of resolved sources in the Gaia DR2 catalog (Tables 4 and 5) is bracketed.

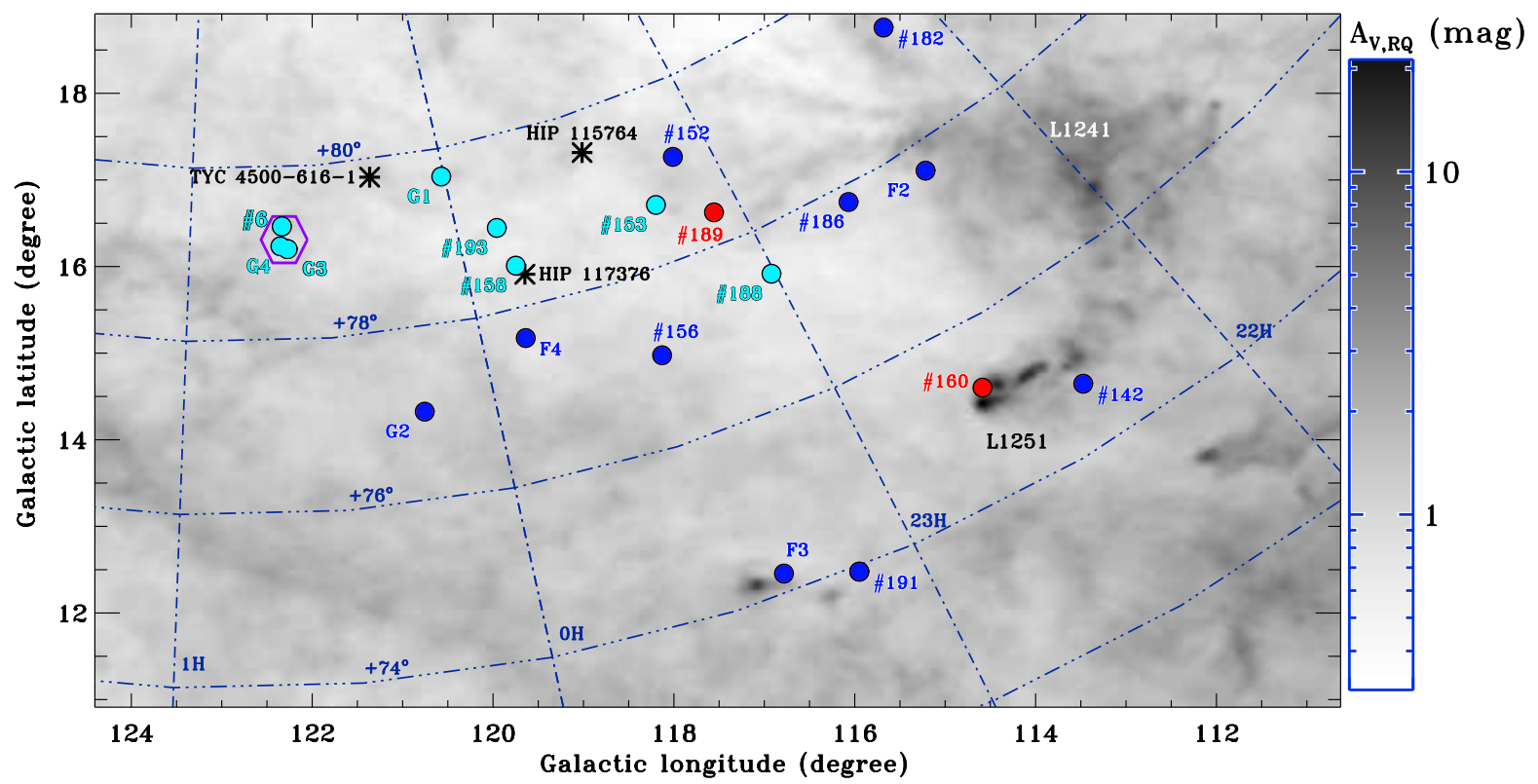

Fig. 10. Spatial distribution of the 20 PMS stars (circles) in the sky area towards the CO Cepheus void, overplotted on the visible extinction $A_{V, \text { RQ }}$ map of Planck Collaboration XXIX (2016) ${ }^{7}$. We display the Cep III members as blue circles and those still gravitationally bound (i.e., Cep II that is a subgroup of Cep III, see Sect. 4.2) as cyan circles, while the non-members are shown in red. The big hexagon encloses the Cep I members. The asterisks mark the locus of the three additional members reported in Oh et al. (2017) and Faherty et al. (2018), as described in Sect. 4.4.

gular area covering Galactic longitude $l$ ranging from $114^{\circ}$ to $126^{\circ}$ and Galactic latitude $b$ from 8.5 to 20.5 , together with the same cut in $K_{s}$ magnitude that we had applied to build the Gaia-2MASS sample. This region slightly differs from the area covered by the selected Gaia-2MASS sources but both are consistent in terms of number of sources. The distributions of distance and proper motions are well reproduced by the simulation for both cases of the maximum distance threshold considered here (400 and 1000 pc; Fig. 12). Therefore, the asymmetry seen in the distribution of the $\mu_{\alpha} \cos \delta$ values is likely due to largescale dynamical processes in the Galaxy rather than the result of the unknown supernova shock expected to be at the origin of the CO Cepheus void (Grenier et al. 1989). Thus, this excess alone cannot be used as a criterion to identify possible new members in this young association and a deeper analysis is required.

To this end, we compared the density map obtained from the Gaia-2MASS sources and the Besançon model (Fig. 13). While the $\left(\mu_{\delta}\right.$, distance) diagram looks the same for the 

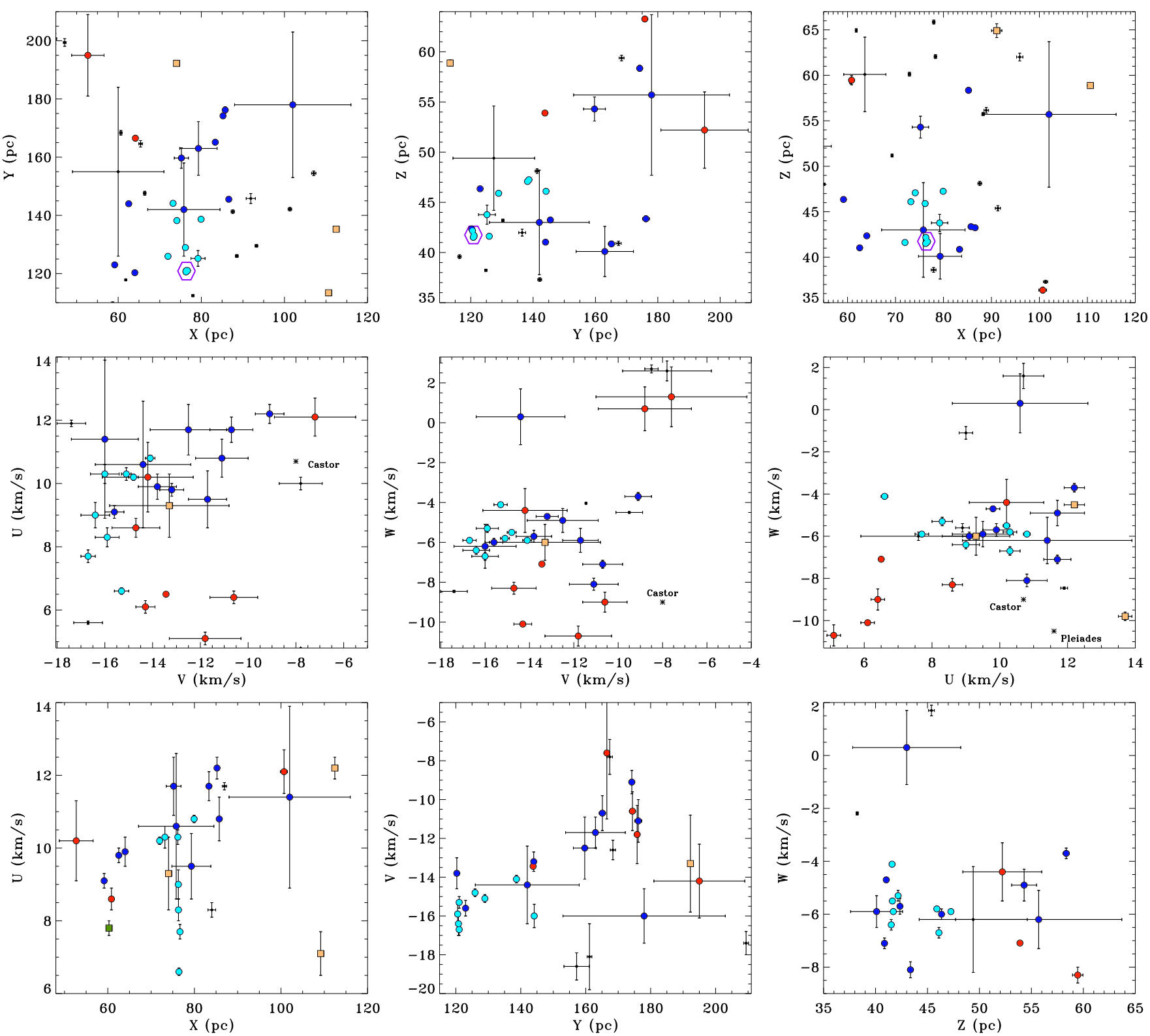

Fig. 11. Same diagrams as in Fig. 8 but covering only the region populated by the PMS-like stars towards the CO Cepheus void. In all the panels, we display the stars composing Cep III and its subgroup (i.e., Cep II) as blue and cyan circles, respectively. The red circles, the orange squares, and black diamonds correspond to the PMS-like that are non-members of Cep III, Pleiades-like stars, and older sources, respectively. In the upper panels, the big open hexagon marks the locus of the smaller concentration (i.e., Cep I).

Gaia-2MASS distribution and the Besançon model, the $\left(\mu_{\alpha} \cos \delta\right.$, distance) diagram reveals a clear over-density in the Gaia-2MASS sample (delimited by the polygonal shape). Coupled to the location of members of the Cepheus association on this density map (Fig. 14), it is indisputable that the excess Gaia2MASS sources are attributable to the young stars in this region. These are the best member candidates to the Cepheus association to investigate with future observations.

\subsection{Cepheus association and runaway stars}

Klutsch (2008) discovered an excess of stellar X-ray sources in Cepheus where Tachihara et al. (2005), and Papers II and III had identified together 15 young stars belonging to the association. The current study has added 14 new members (i.e., six PMS stars and eight comoving companions of these 21 young stars).
All but two PMS stars towards the CO Cepheus void have the same kinematic properties (Table 6) and therefore a common origin. We also found that eight of the young stars in Tachihara et al. (2005) belong to this association, confirming their assumption. The discovery of such a large number of young stars in this region is more easily explained by the insitu model than by the runaway hypothesis. As mentioned by Tachihara et al. (2005) and Paper II, these sources are close to a faint, low density cloud that could be the remnant of the parent cloud already dissipated.

We see a decline in multiple systems and a shift from the mean Galactic position $Y$ to positive values in the more scattered group (Cep III) with respect to the densest group (Cep I). This agrees with the dispersion of the cluster members driven by the rotation of the Galaxy. This also explains the gradient found in the $\left(\mu_{\alpha} \cos \delta\right.$, distance) diagram (Fig. 14) because 

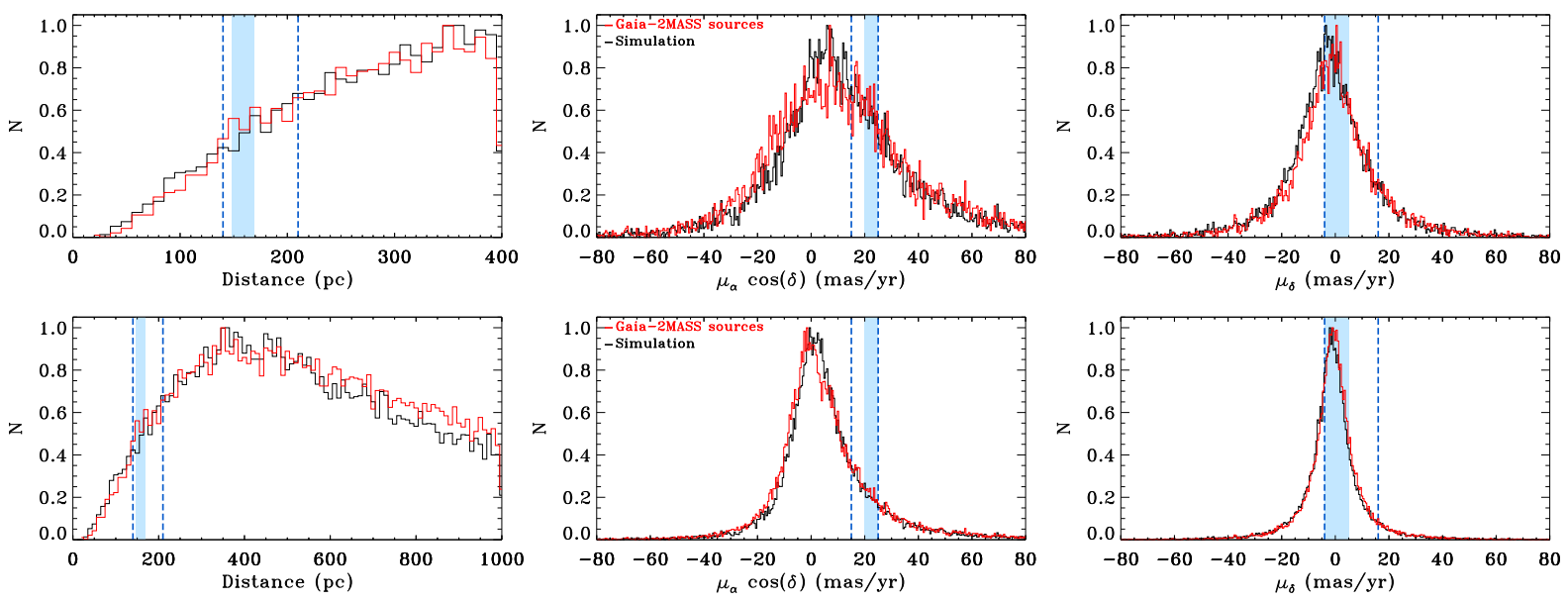

Fig. 12. Comparison of the distribution of distance (left panels), and proper motion in right ascension (middle panels) and in declination (right panels) between the Gaia-2MASS sources and the simulation with the Besançon model (Sect. 4.3). We normalized the number of stars in each bin, dividing by the maximum number of sources obtained for each histogram. For these plots, we only considered all the sources having a distance lower than $400 \mathrm{pc}$ (upper panels) and $1000 \mathrm{pc}$ (lower panels). In each panel, the blue-shaded area denotes the region populated by the likely members of Cep II, while the dashed lines correspond to those belonging to Cep III.
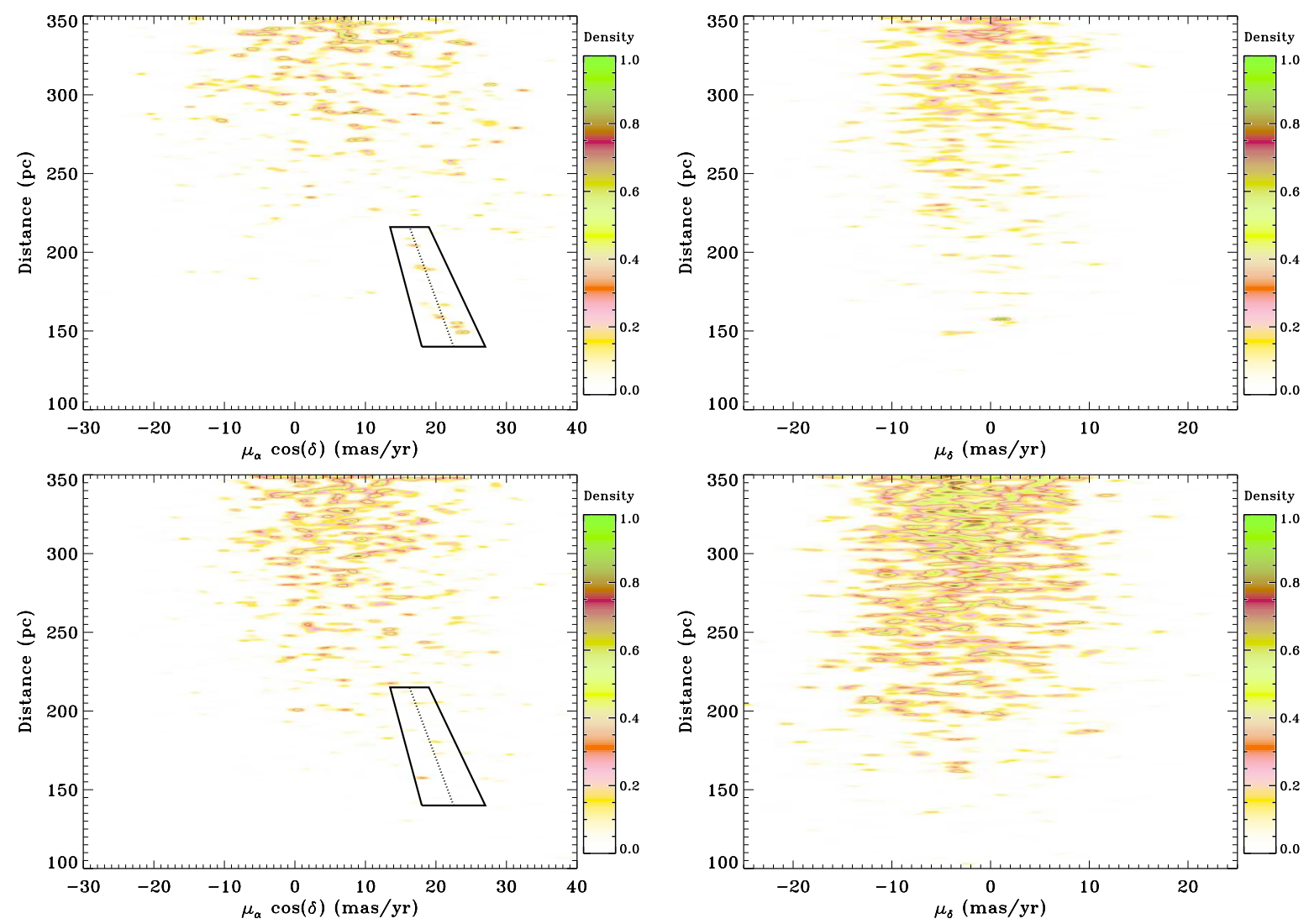

Fig. 13. Comparison of the density map obtained from the distribution of distance as a function of the proper motion in right ascension (left panels) and in declination (right panels), between the Gaia-2MASS sources (upper panels) and the simulation with the Besançon model (lower panels). Left panels: the polygonal shape denotes the region populated by the PMS-like stars belonging to Cep III.

the members of the most extended stellar group tend to move further away from the others. Based on Table 6, the properties of the three stellar groups could be interpreted as follows: (i) the strongest concentration of young stars (Cep I) could be the remnant of the past Cepheus cluster core, (ii) the sources belonging to the group Cep II could correspond to the members that are still gravitationally bound, and (iii) the most extended group (Cep III) denotes the original cluster.
The latter corresponds to the Cepheus association reported in Paper II.

The group 38 of Oh et al. (2017) and Faherty et al. (2018) actually coincides with the Cepheus association analyzed here and lists three additional members (HIP 115764, HIP 117376, and TYC 4500-616-1). Their astrometries and the $R V$ value of TYC 4500-616-1 in Gaia DR2 perfectly agree with those of the Cep II members (Fig. 14), while the $R V$ value of HIP 117376 


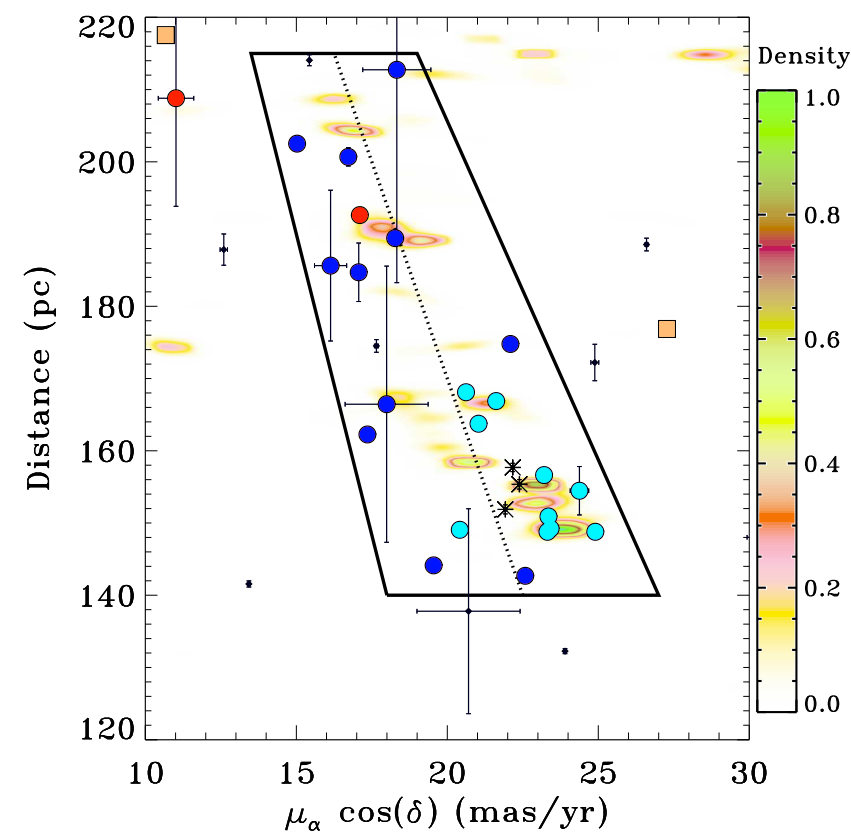

Fig. 14. ( $\mu_{\alpha} \cos \delta$, distance) diagram of our young star candidates located in the region populated by the members of the Cepheus association, overplotted on the Gaia-2MASS density map (Fig. 13). We use the same symbols as in Fig. 11. The asterisks mark the locus of additional members reported by Oh et al. (2017) and Faherty et al. (2018): HIP 115764, HIP 117376, and TYC 4500-616-1 (from bottom to top).

suggests a possible variation in radial velocity. The source HIP 115764 would be the warmest member of the association with an effective temperature of $8020_{-140}^{+420} \mathrm{~K}$ in Gaia DR2. We also note that the angular separation between HIP 117376 and our target \#158 is about $8.30 \pm 0.044$ mas. This brings the number of current bona fide members to 32 .

We discovered six PMS stars outside the CO Cepheus void (\#93, \#106 c1, \#115, \#124, \#150, and F1) with space-velocity components similar to the members of the Cepheus association. These correspond to the red circles in the $(U, W)$ plane of Fig. 11 . Their distances range from 160 to $240 \mathrm{pc}$, except for \#150 (distance $=375 \pm 3 \mathrm{pc}$ ). The five remaining sources (and their two comoving companions; Table 5) could be runaway stars. This list of sources includes one of the PMS stars from Paper III and one WTTS from Tachihara et al. (2005).

\section{Conclusions}

We present the results of a spectroscopic survey of optical counterparts of X-ray sources in the Cepheus region (near the North Celestial pole) aimed at discovering further young objects around the four comoving stars reported by us in Paper II. Based on multivariate analysis methods, we selected optical and infrared counterparts of ROSAT All-Sky Survey and XMMNewton X-ray sources, which are young star candidates. The analysis of the spectra of these candidates allowed us to determine their atmospheric parameters, radial and rotational velocities, and atmospheric lithium content. These data, along with the parallaxes and proper motions from the Gaia DR2 catalog, were used to characterize our sample and identify new young stars in this region. The main stellar populations composing this sample are young or active stars and multiple systems. We identified two distinct populations of young stars that are spatially and kinematically separated. The 18 Pleiades-like objects with an age between 100 and $300 \mathrm{Myr}$ are mostly projected towards the Galactic plane, while 23 of the 37 sources younger than $30 \mathrm{Myr}$ are located in the sky area of 8 degree-diameter filling the CO Cepheus void. Among these, 21 PMS stars (including five spectroscopic binaries) and their eight comoving companions belong to the Cepheus association, which is the first nearby (distance $=157 \pm 10 \mathrm{pc}$ ) young (age $=10-20 \mathrm{Myr})$ stellar association found northward of $\delta=+30^{\circ}$.

We provide the first comprehensive view of the Cepheus association. All studies carried out so far in this sky region have found a total of 32 bona fide members and nine member candidates of this young association, to which 14 (44\%) members and six $(67 \%)$ candidates are new discoveries. The kinematics of its members reveals a substantial mixture of the original cluster within the local population of the Galactic plane. The runaway hypothesis is highly improbable for explaining the formation of this homogeneous stellar group because of their kinematic properties and the identification of several new members. This raises the question of the in-situ star-formation scenario in low-mass cloud environments (as in many other SFRs).

Coupled to the Cepheus association properties derived from the current analysis, our Gaia-2MASS sample should contribute to the identification of new candidates to be followed up in the future.

Acknowledgements. We thank the anonymous referee for useful suggestions. This work is supported by the Universidad Complutense de Madrid and Spanish Ministerio de Ciencia e Innovación y Universidades (MICINN) under grant AYA2016-79425-C3-1-P. A.K. and D.M. were also supported by AstroMadrid (CAM S2009/ESP-1496), and MICINN under grants AYA2008-00695 and AYA2008-06423-C03-03. Part of this study is also supported by the Italian Ministero dell'Istruzione, Università e Ricerca (MIUR), the French Centre National d'Études spatiales (CNES), and the Région Alsace. This research made use of the SIMBAD database, the VIZIER catalog access and the X-Match service, which are operated at the Centre de Données astronomiques de Strasbourg (CDS). This publication makes use of the data products from ROSAT and XMM-Newton X-ray observatories, the Two Micron All Sky Survey, and the European Space Agency (ESA) missions Gaia and Planck. The ROSAT All-Sky Survey catalogs were produced by the ROSAT Scientific Data Center at the MaxPlanck-Institut für Extraterrestrische Physik (MPE), Garching (Germany). The 2XMMi-DR3 catalog is the fifth publicly released XMM-Newton X-ray source catalog produced by the XMM-Newton Survey Science Centre (SSC) consortium on behalf of ESA. The 2XMMi-DR3 is one of two incremental versions of the 2XMM catalog. The Two Micron All Sky Survey, which is a joint project of the University of Massachusetts and the Infrared Processing and Analysis Center/California Institute of Technology, was funded by the National Aeronautics and Space Administration and the National Science Foundation. The data from the ESA mission Gaia (https: //www. cosmos. esa.int/gaia) was processed by the Gaia Data Processing and Analysis Consortium (DPAC, https://www. cosmos.esa.int/web/gaia/dpac/consortium). Funding for the DPAC has been provided by national institutions, in particular the institutions participating in the Gaia Multilateral Agreement. Planck (http://www.esa.int/Planck) is an ESA science mission with instruments and contributions directly funded by ESA Member States, NASA, and Canada. This publication used the POLLUX database (http: //pollux.graal . univ-montp2 . fr) operated at LUPM (Laboratoire Univers et Particules de Montpellier, Université Montpellier II CNRS, France) with the support of the French Programme National de Physique Stellaire and Institut national des sciences de l'Univers.

\section{References}

Abt, H. A. 2008, ApJS, 176, 216

Abt, H. A., \& Willmarth, D. 2006, ApJS, 162, 207

Alcala, J. M., Krautter, J., Covino, E., et al. 1997, A\&A, 319, 184

Alonso-Floriano, F. J., Morales, J. C., Caballero, J. A., et al. 2015, A\&A, 577, A128

Barden, S. C. 1985, ApJ, 295, 162

Bertout, C., Siess, L., \& Cabrit, S. 2007, A\&A, 473, L21

Bessell, M. S., \& Brett, J. M. 1988, PASP, 100, 1134

Bouchy, F., Hébrard, G., Udry, S., et al. 2009, A\&A, 505, 853

Bouvier, J., Barrado, D., Moraux, E., et al. 2018, A\&A, 613, A63 
Bruntt, H., Bedding, T. R., Quirion, P.-O., et al. 2010, MNRAS, 405, 1907

Caballero, J. A., Cortés-Contreras, M., Alonso-Floriano, F. J., et al. 2013 Protostars and Planets VI Posters, 20

Carpenter, J. M. 2001, AJ, 121, 2851

Casagrande, L., Ramírez, I., Meléndez, J., Bessell, M., \& Asplund, M. 2010 A\&A, 512, A54

Casagrande, L., Schönrich, R., Asplund, M., et al. 2011, A\&A, 530, A138

Cowley, A. P., \& Bidelman, W. P. 1979, PASP, 91, 83

Cutri, R. M., Wright, E. L., Conrow, T., et al. 2012, Explanatory Supplement to the WISE All-Sky Data Release Products, Tech. rep.

Czekaj, M. A., Robin, A. C., Figueras, F., Luri, X., \& Haywood, M. 2014, A\&A, $564, \mathrm{~A} 102$

da Silva, L., Torres, C. A. O., de La Reza, R., et al. 2009, A\&A, 508, 833

da Silva, R., Milone, A. C., \& Reddy, B. E. 2011, A\&A, 526, A71

Daemgen, S., Siegler, N., Reid, I. N., \& Close, L. M. 2007, ApJ, 654, 558

Dame, T. M., Hartmann, D., \& Thaddeus, P. 2001, ApJ, 547, 792

Delfosse, X., Forveille, T., Perrier, C., \& Mayor, M. 1998, A\&A, 331, 581

Dobashi, K., Uehara, H., Kandori, R., et al. 2005, PASJ, 57, 1

Draine, B. T., \& Li, A. 2007, ApJ, 657, 810

Droege, T. F., Richmond, M. W., Sallman, M. P., \& Creager, R. P. 2006, PASP, 118,1666

Eggen, O. J. 1996, AJ, 112, 1595

ESA 1997, in The HIPPARCOS and TYCHO Catalogues. Astrometric and Photometric Star Catalogues Derived from the ESA HIPPARCOS Space Astrometry Mission, ESA Spec. Publ., 1200

Faherty, J. K., Bochanski, J. J., Gagné, J., et al. 2018, ApJ, 863, 91

Famaey, B., Jorissen, A., Luri, X., et al. 2005, A\&A, 430, 165

Feigelson, E. D. 1996, ApJ, 468, 306

Finch, C. T., \& Zacharias, N. 2016, VizieR Online Data Catalog: I/333

Fischer, D. A., \& Valenti, J. 2005, ApJ, 622, 1102

Fleming, T. A., Liebert, J., Gioia, I. M., \& Maccacaro, T. 1988, ApJ, 331, 958

Flesch, E. 2010, PASA, 27, 283

Frasca, A., \& Catalano, S. 1994, A\&A, 284, 883

Frasca, A., Catalano, S., \& Mantovani, D. 1997, A\&A, 320, 101

Frasca, A., Freire Ferrero, R., Marilli, E., \& Catalano, S. 2000, A\&A, 364, 179

Frasca, A., Guillout, P., Marilli, E., et al. 2006, A\&A, 454, 301

Frasca, A., Covino, E., Spezzi, L., et al. 2009, A\&A, 508, 1313

Frasca, A., Biazzo, K., Lanzafame, A. C., et al. 2015, A\&A, 575, A4

Frasca, A., Guillout, P., Klutsch, A., et al. 2018, A\&A, 612, A96

Fuhrmann, K. 2008, MNRAS, 384, 173

Gagné, J., \& Faherty, J. K. 2018, ApJ, 862, 138

Gagné, J., Lafrenière, D., Doyon, R., Malo, L., \& Artigau, É. 2014, ApJ, 783,

Gagné, J., Faherty, J. K., Cruz, K. L., et al. 2015a, ApJS, 219, 33

Gagné, J., Lafrenière, D., Doyon, R., Malo, L., \& Artigau, É. 2015b, ApJ, 798,

Gagné, J., Mamajek, E. E., Malo, L., et al. 2018a, ApJ, 856, 23

Gagné, J., Roy-Loubier, O., Faherty, J. K., Doyon, R., \& Malo, L. 2018b, ApJ, 860,43

Gaia Collaboration (Prusti, T., et al.) 2016, A\&A, 595, A1

Gaia Collaboration (Brown, A. G. A., et al.) 2018, A\&A, 616, A

Gigoyan, K. S., \& Mickaelian, A. M. 2012, MNRAS, 419, 3346

Gilmore, G., Randich, S., Asplund, M., et al. 2012, The Messenger, 147, 25

Girardi, L., Bressan, A., Bertelli, G., \& Chiosi, C. 2000, A\&AS, 141, 37

Glebocki, R., \& Gnacinski, P. 2005, VizieR Online Data Catalog: III/244

Gontcharov, G. A. 2006, Astron. Lett., 32, 759

Gonzalez, G., Carlson, M. K., \& Tobin, R. W. 2010, MNRAS, 403, 1368

Gorbikov, E., \& Brosch, N. 2014, MNRAS, 443, 725

Gorti, U., \& Bhatt, H. C. 1996, MNRAS, 278, 611

Gray, R. O., Napier, M. G., \& Winkler, L. I. 2001, AJ, 121, 2148

Gray, R. O., Corbally, C. J., Garrison, R. F., McFadden, M. T., \& Robinson, P. E. 2003, AJ, 126, 2048

Gray, R. O., Corbally, C. J., Garrison, R. F., et al. 2006, AJ, 132, 161

Grenier, I. A., Lebrun, F., Arnaud, M., Dame, T. M., \& Thaddeus, P. 1989, ApJ, 347,231

Guillout, P., Sterzik, M. F., Schmitt, J. H. M. M., et al. 1998a, A\&A, 334, 540

Guillout, P., Sterzik, M. F., Schmitt, J. H. M. M., Motch, C., \& Neuhaeuser, R. 1998b, A\&A, 337, 113

Guillout, P., Schmitt, J. H. M. M., Egret, D., et al. 1999, A\&A, 351, 1003

Guillout, P., Klutsch, A., Frasca, A., et al. 2009, A\&A, 504, 829

Guillout, P., Frasca, A., Klutsch, A., Marilli, E., \& Montes, D. 2010, A\&A, 520, A94

Harlan, E. A. 1969, AJ, 74, 916

Hauschildt, P. H., Allard, F., \& Baron, E. 1999, ApJ, 512, 377

Hekker, S., \& Meléndez, J. 2007, A\&A, 475, 1003

Henden, A. A., Levine, S., Terrell, D., \& Welch, D. L. 2015, Am. Astron. Soc. Meet. Abstr., 225, 336.16

Herbig, G. H. 1985, ApJ, 289, 269
Herrero, E., Ribas, I., Jordi, C., Guinan, E. F., \& Engle, S. G. 2012, A\&A, 537, A147

Høg, E., Fabricius, C., Makarov, V. V., et al. 2000, A\&A, 355, L27

Houdebine, E. R. 2010, MNRAS, 407, 1657

Houdebine, E. R. 2011, MNRAS, 416, 2233

Janson, M., Hormuth, F., Bergfors, C., et al. 2012, ApJ, 754, 44

Jeffers, S. V., Schöfer, P., Lamert, A., et al. 2018, A\&A, 614, A76

Jeffries, R. D., Pallavicini, R., Micela, G., \& Sciortino, S. 2000, in Stellar Clusters and Associations: Convection, Rotation, and Dynamos, ASP Conf. Ser., 198, 245

Jeffries, R. D. 2017, Mem. Soc. Astron. It., 88, 637

Jeffries, R. D., Jackson, R. J., Cottaar, M., et al. 2014, A\&A, 563, A94

Jenkins, J. S., Ramsey, L. W., Jones, H. R. A., et al. 2009, ApJ, 704, 975

Jiménez-Esteban, F. M., Caballero, J. A., Dorda, R., Miles-Páez, P. A., \& Solano, E. 2012, A\&A, 539, A86

Kharchenko, N. V., Scholz, R.-D., Piskunov, A. E., Röser, S., \& Schilbach, E. 2007, Astron. Nachr., 328, 889

Kiss, Z. T., Tóth, L. V., Krause, O., Kun, M., \& Stickel, M. 2006, A\&A, 453, 923

Klutsch, A. 2008, PhD Thesis, Observatoire de Strasbourg, Universié Louis Pasteur

Klutsch, A., Frasca, A., Guillout, P., et al. 2008, A\&A, 490, 737

Klutsch, A., Alonso-Floriano, F. J., Caballero, J. A., et al. 2012, in SF2A-2012: Proceedings of the Annual meeting of the French Society of Astronomy and Astrophysics, eds. S. Boissier, P. de Laverny, N. Nardetto, et al., 357

Klutsch, A., Freire Ferrero, R., Guillout, P., et al. 2014, A\&A, 567, A52

Kun, M. 2008, in Handbook of Star Forming Regions, Volume I, ed. B. Reipurth (Astronomical Society of the Pacific Monograph Publications), 240

Kun, M., \& Prusti, T. 1993, A\&A, 272, 235

Kun, M., Kiss, Z. T., \& Balog, Z. 2008, in Handbook of Star Forming Regions, Volume I, ed. B. Reipurth (Astronomical Society of the Pacific Monograph Publications), 136

Kun, M., Balog, Z., Kenyon, S. J., Mamajek, E. E., \& Gutermuth, R. A. 2009, ApJS, 185, 451

Lasker, B. M., Lattanzi, M. G., McLean, B. J., et al. 2008, AJ, 136, 735

Lee, S.-G. 1984, AJ, 89, 702

Lépine, S., \& Shara, M. M. 2005, AJ, 129, 1483

Lépine, S., Hilton, E. J., Mann, A. W., et al. 2013, AJ, 145, 102

Luri, X., Palmer, M., Arenou, F., et al. 2014, A\&A, 566, A119

Makarov, V. V., Zacharias, N., Hennessy, G. S., Harris, H. C., \& Monet, A. K. B. 2007, ApJ, 668, L155

Malo, L., Doyon, R., Lafrenière, D., et al. 2013, ApJ, 762, 88

Mapelli, M., Vallenari, A., Jeffries, R. D., et al. 2015, A\&A, 578, A35

McCarthy, K., \& Wilhelm, R. J. 2014, AJ, 148, 70

Meléndez, J., Asplund, M., Alves-Brito, A., et al. 2008, A\&A, 484, L21

Mishenina, T. V., Soubiran, C., Bienaymé, O., et al. 2008, A\&A, 489, 923

Monet, D. G., Levine, S. E., Canzian, B., et al. 2003, AJ, 125, 984

Montes, D., Fernandez-Figueroa, M. J., de Castro, E., \& Cornide, M. 1995, A\&AS, 109, 135

Montes, D., López-Santiago, J., Gálvez, M. C., et al. 2001, MNRAS, 328, 45

Moór, A., Pascucci, I., Kóspál, Á., et al. 2011, ApJS, 193, 4

Motch, C., Guillout, P., Haberl, F., et al. 1997a, A\&AS, 122, 201

Motch, C., Haberl, F., Dennerl, K., Pakull, M., \& Janot-Pacheco, E. 1997b, A\&A, 323,853

Motch, C., Guillout, P., Haberl, F., et al. 1998, A\&AS, 132, 341

Moultaka, J., Ilovaisky, S. A., Prugniel, P., \& Soubiran, C. 2004, PASP, 116, 693

Nidever, D. L., Marcy, G. W., Butler, R. P., Fischer, D. A., \& Vogt, S. S. 2002, ApJS, 141, 503

Nissen, P. E., \& Schuster, W. J. 2010, A\&A, 511, L10

Oh, S., Price-Whelan, A. M., Hogg, D. W., Morton, T. D., \& Spergel, D. N. 2017, AJ, 153, 257

Palacios, A., Gebran, M., Josselin, E., et al. 2010, A\&A, 516, A13

Pavlenko, Y. V., \& Magazzu, A. 1996, A\&A, 311, 961

Perruchot, S., Kohler, D., Bouchy, F., et al. 2008, in Presented at the Society of Photo-Optical Instrumentation Engineers (SPIE) Conference, SPIE Conf. Ser., 7014

Pfeiffer, M. J., Frank, C., Baumueller, D., Fuhrmann, K., \& Gehren, T. 1998, A\&AS, 130, 381

Pineau, F. X. 2009, PhD Thesis, Observatoire de Strasbourg, Universié de Strasbourg

Pineau, F. X., Derriere, S., Michel, L., \& Motch, C. 2008a, in Astronomical Data Analysis Software and Systems XVII, eds. R. W. Argyle, P. S. Bunclark, \& J. R. Lewis, ASP Conf. Ser., 394, 369

Pineau, F. X., Derriere, S., Michel, L., \& Motch, C. 2008b, in American Institute of Physics Conference Series, ed. C. A. L. Bailer-Jones, 1082, 15

Pineau, F.-X., Motch, C., Carrera, F., et al. 2011, A\&A, 527, A126

Piskunov, A. E., Kharchenko, N. V., Schilbach, E., et al. 2008, A\&A, 487, 557

Planck Collaboration XXIX. 2016, A\&A, 586, A132 
Preibisch, T., Kim, Y.-C., Favata, F., et al. 2005, ApJS, 160, 401

Ramírez, I., Allende Prieto, C., \& Lambert, D. L. 2007, A\&A, 465, 271

Randich, S., Gilmore, G., \& Gaia-ESO Consortium 2013, The Messenger, 154 47

Reddy, B. E., \& Lambert, D. L. 2008, MNRAS, 391, 95

Robin, A. C., Luri, X., Reylé, C., et al. 2012, A\&A, 543, A100

Roeser, S., Demleitner, M., \& Schilbach, E. 2010, AJ, 139, 2440

Sacco, G. G., Jeffries, R. D., Randich, S., et al. 2015, A\&A, 574, L7

Shkolnik, E., Liu, M. C., \& Reid, I. N. 2009, ApJ, 699, 649

Shkolnik, E. L., Hebb, L., Liu, M. C., Reid, I. N., \& Collier Cameron, A. 2010, ApJ, 716, 1522

Siess, L., Dufour, E., \& Forestini, M. 2000, A\&A, 358, 593

Simon, T. 2006, AJ, 131, 501

Simon, T. 2009, ApJ, 694, 425

Skiff, B. A. 2010, VizieR Online Data Catalog: B/mk/mktypes

Skrutskie, M. F., Cutri, R. M., Stiening, R., et al. 2003, VizieR Online Data Catalog: VII/233

Skrutskie, M. F., Cutri, R. M., Stiening, R., et al. 2006, AJ, 131, 1163

Soderblom, D. R., Jones, B. F., Balachandran, S., et al. 1993a, AJ, 106, 1059

Soderblom, D. R., Fedele, S. B., Jones, B. F., Stauffer, J. R., \& Prosser, C. F. 1993b, AJ, 106, 1080

Soderblom, D. R., King, J. R., \& Henry, T. J. 1998, AJ, 116, 396

Soubiran, C., Katz, D., \& Cayrel, R. 1998, A\&AS, 133, 221

Soubiran, C., Jasniewicz, G., Chemin, L., et al. 2013, A\&A, 552, A64

Soubiran, C., Jasniewicz, G., Chemin, L., et al. 2018, A\&A, 616, A7

Soubiran, C., Le Campion, J. F., Cayrel de Strobel, G., \& Caillo, A. 2010, A\&A 515, A111

Stassun, K. G., Oelkers, R. J., Pepper, J., et al. 2018, AJ, 156, 102
Stephenson, C. B. 1986, AJ, 91, 144

Sterzik, M. F., \& Durisen, R. H. 1995, A\&A, 304, L9

Sterzik, M. F., \& Durisen, R. H. 1998, A\&A, 339, 95

Sterzik, M. F., Alcala, J. M., Neuhaeuser, R., \& Schmitt, J. H. M. M. 1995, A\&A, 297,418

Tachihara, K., Neuhäuser, R., Kun, M., \& Fukui, Y. 2005, A\&A, 437, 919

Tonry, J., \& Davis, M. 1979, AJ, 84, 1511

Torres, C. A. O., Quast, G. R., da Silva, L., et al. 2006, A\&A, 460, 695

Torres, C. A. O., Quast, G. R., Melo, C. H. F., \& Sterzik, M. F. 2008, in Handbook of Star Forming Regions, Volume II, ed. B. Reipurth (Astronomical Society of the Pacific Monograph Publications), 757

Valenti, J. A., \& Fischer, D. A. 2005, ApJS, 159, 141

Voges, W., Aschenbach, B., Boller, T., et al. 1999, A\&A, 349, 389

Voges, W., Aschenbach, B., Boller, T., et al. 2000, IAU Circ., 7432, 3

Vyssotsky, A. N. 1956, AJ, 61, 201

Wang, S., \& Chen, X. 2019, ApJ, 877, 116

Watson, M. G., Schröder, A. C., Fyfe, D., et al. 2009, A\&A, 493, 339

West, A. A., Hawley, S. L., Bochanski, J. J., et al. 2008, AJ, 135, 785

Woolf, V. M., \& Wallerstein, G. 2005, MNRAS, 356, 963

Wright, E. L., Eisenhardt, P. R. M., Mainzer, A. K., et al. 2010, AJ, 140, 1868

Zacharias, N., Monet, D. G., Levine, S. E., et al. 2004, in American Astronomical Society Meeting Abstracts, BAAS, 36, 1418

Zacharias, N., Finch, C., Subasavage, J., et al. 2015, AJ, 150, 101

Zapatero Osorio, M. R., Béjar, V. J. S., Pavlenko, Y., et al. 2002, A\&A, 384, 937

Zinnecker, H. 2008, Workshop on Star Formation Across the Milky Way Galaxy (ESO)

Zuckerman, B., \& Song, I. 2004, ARA\&A, 42, 685

Zuckerman, B., Rhee, J. H., Song, I., \& Bessell, M. S. 2011, ApJ, 732, 61 


\section{Appendix A: Extraction of spectra of binary-pair candidates with a double Gaussian profile}

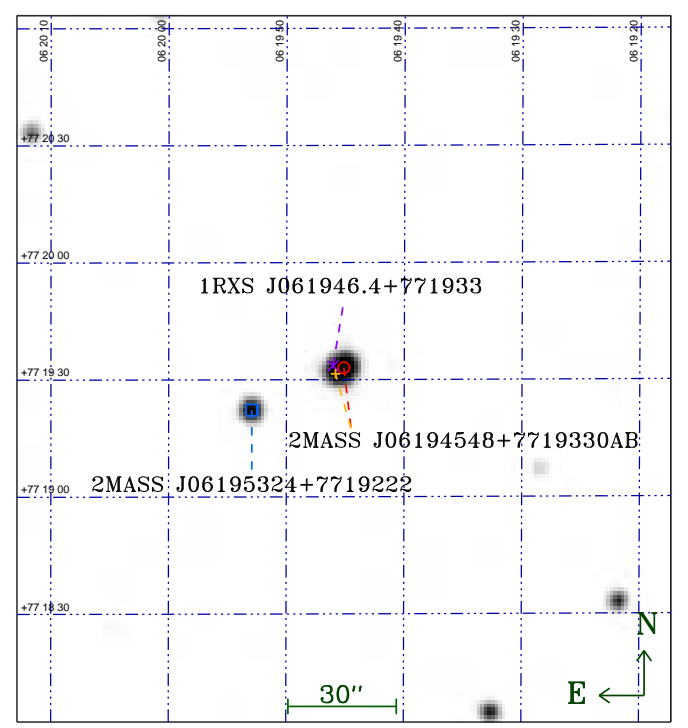

Fig. A.1. 2MASS $K_{s}$-band image (Epoch J1999.10), centered on the source 2MASS J06194548+7719330 (circle) and its nearest companion Gaia DR2 1116789735748819968 ("plus" symbol). We also show the positions of the X-ray source 1RXS J061946.4+771933 (cross) and the source 2MASS J06195324+7719222 (square).

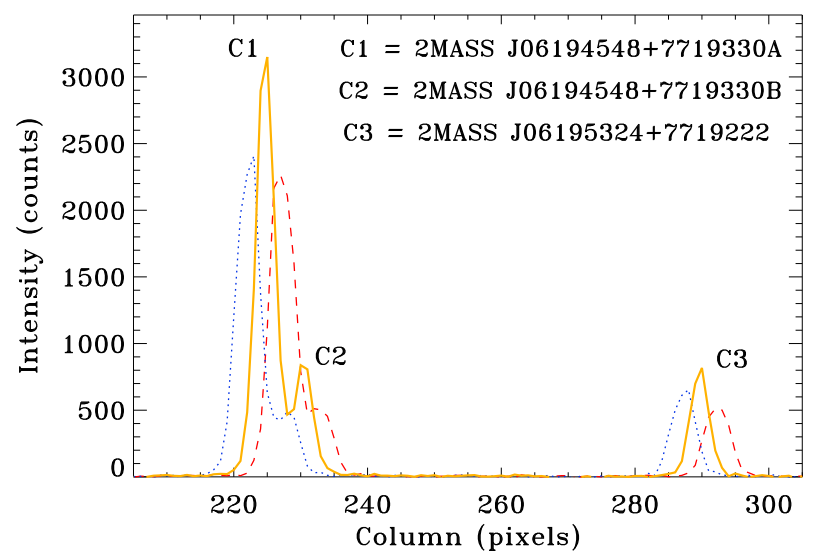

Fig. A.2. Line numbers 1300 (red dashed line), 2000 (orange solid line), and 2700 (blue dotted line), perpendicular to the spectral direction. The three apertures $\mathrm{C} 1-\mathrm{C} 3$ are clearly visible.

To benefit from the IDS instrumental setup, we sought to identify possible binary-pair candidates within our target list before starting our observing runs. However, during the observations themselves, we found additional likely visual binaries having a lower angular separation. We did not identify them previously because the resolution of public images is not enough to resolve such binaries with sufficient confidence, before the launch of Gaia observatory. In such cases, we oriented the slit with the position angle of the two components to simultaneously image them. Hereafter, we illustrate this work on the source \#40 (=1RXS J061946.4+771933) because the alignment of three sources allows us to validate our work process.

The source 2MASS J06194548+7719330 seems to have a weakly-elongated shape with respect to that of nearby stars (Fig. A.1), which is typical of unresolved binaries and sources with a low angular separation. During our observing runs

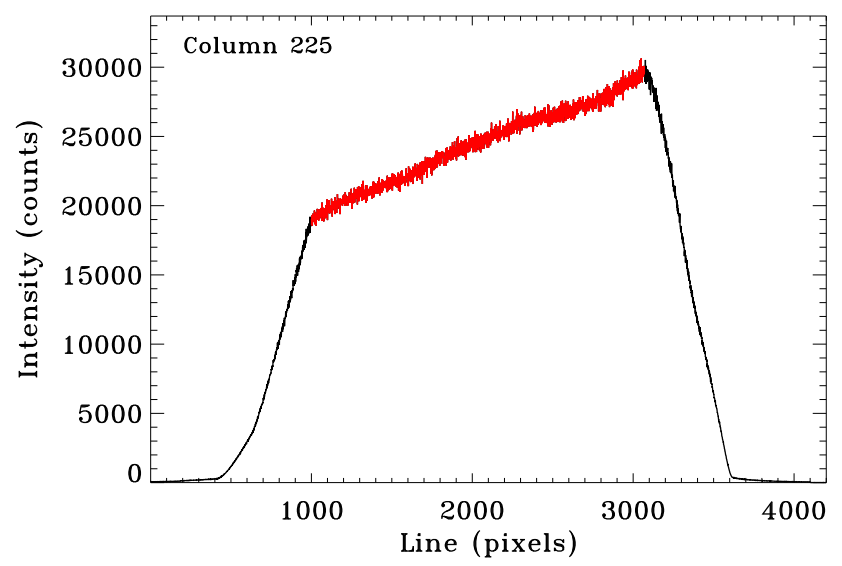

Fig. A.3. Lamp flat on the $235 \mathrm{~mm}$ camera with the $2 k \times 4 k$ RED +2 CCD detector mounted on IDS. The portion in red corresponds to the approximately $2070 \mathrm{CCD}$ pixels that are clear and unvignetted.

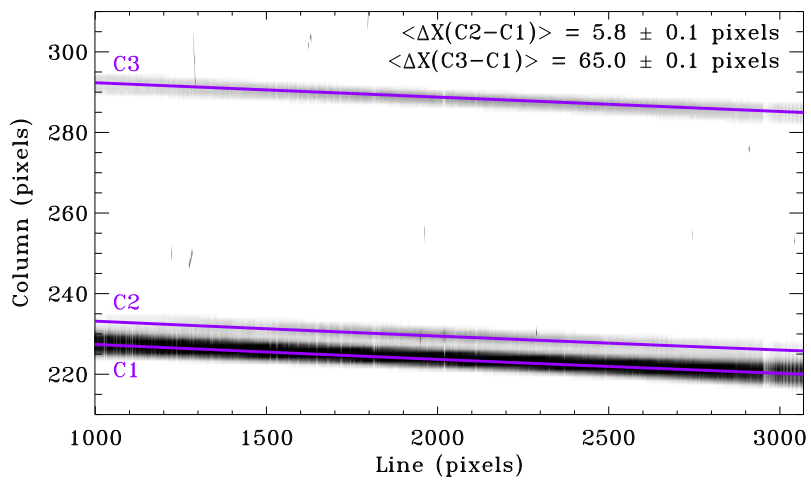

Fig. A.4. Zoom on the section of the image where the traces of the three sources can be used for a spectral analysis. This corresponds to all the clear and unvignetted lines (Fig. A.3) and columns from 205 to 305 . We also overplotted the trace (solid lines) obtained for each of the three sources. On the right upper corner, we indicate the mean separation between the main aperture $(\mathrm{C} 1)$ and the two others $(\mathrm{C} 2$ and $\mathrm{C} 3)$.

because we actually saw two sources close to the coordinates of our candidate. These turn out to be comoving stars (Table 5). To position the slit on these two objects, we then set the slit position angle to about $114^{\circ}$. Based on the Gaia DR2 coordinates, this angle is rather similar to the position angle $\theta$ of $115.13 \pm 24^{\prime \prime \prime 35}$ between these two sources, and of $114.44 \pm 2$ '.95 between the source \#40 and 2MASS J06195324+7719222. On the cuts perpendicular to the spectral direction, the aperture of the three components are clearly visible, regardless of the line used, even if the apertures $\mathrm{C} 1$ and $\mathrm{C} 2$ are partially blended (Fig. A.2).

We firstly used the procedure of extraction within the IRAF environment, APALL. As the spectrum obtained from the second aperture $(\mathrm{C} 2)$ was contaminated by the light of the bright source (C1), we needed to perform an extraction by means of a double Gaussian profile in order to accurately fit each trace and thus substantially reduce such a contamination. In this context, we adapted the code of Frasca et al. (1997) to the IDS data. As the $235 \mathrm{~mm}$ camera optics severely vignettes the outer regions of the dispersed light beam, only 2070 of the CCD pixels are useful for the H1800V dispersion grating (Fig. A.3). We determined the shape of each aperture by fitting all the perpendicular cuts along the spectral direction of clear and unvignetted lines. We extracted the two spectra from the partially blended apertures $\mathrm{C} 1$ and $\mathrm{C} 2$ using a double Gaussian profile, while we fitted the third aperture 
(C3) with an additional simple Gaussian profile. The traces for the three sources are displayed on Fig. A.4.

Using the 2MASS, NOMAD, and WISE coordinates, we found a spatial scale of $0.42-0.43 \operatorname{arcsec}$ pixel $^{-1}$. This agrees with the value of $0.44 \operatorname{arcsec}_{\text {pixel }}{ }^{-1}$ tabulated for this detector. From the clear and unvignetted lines, we found an average gap of $5.8 \pm 0.1$ and $65.0 \pm 0.1$ pixels between the main aperture $(\mathrm{C} 1)$ and the two others ( $\mathrm{C} 2$ and $\mathrm{C} 3$, respectively). These correspond to an angular separation $\rho$ of $22^{\prime \prime} 49 \pm 0 \prime \prime 07$ and $28^{\prime \prime} \cdot 0 \pm 0$ 0 $0^{\prime} 4$. These are consistent with the angular separation derived from the 2MASS coordinates $\left(\rho_{C_{1}-C_{3}}=27^{\prime \prime} \cdot 707 \pm 00^{\prime \prime} \cdot 079\right)$ and the Gaia DR2 ones $\left(\rho_{C_{1}-C_{2}}=2^{\prime \prime} .52 \pm 0.032\right.$ mas and $\left.\rho_{C_{1}-C_{3}}=28^{\prime \prime} .39 \pm 0.040 \mathrm{mas}\right)$.

\section{Appendix B: Multiple systems}

Table B.1. Identification of the second source that we have imaged during the acquisition of the IDS spectrum of our target.

\begin{tabular}{ll}
\hline \hline$\#$ & Source name \\
\hline $6 \mathrm{c} 1$ & TYC 4500-1549-1 \\
$6 \mathrm{c} 3$ & Gaia DR2 564707973733134080 \\
$31 \mathrm{c} 1$ & Gaia DR2 475251432820143872 \\
$37 \mathrm{c} 2$ & TYC 4354-793-1 \\
$40 \mathrm{c} 2$ & Gaia DR2 1116789735748819968 \\
$40 \mathrm{c} 3$ & Gaia DR2 1116789667029342080 \\
$42 \mathrm{c} 2$ & Gaia DR2 1116535920362524928 \\
$44 \mathrm{c} 2$ & TYC4618-329-1 \\
$81 \mathrm{c} 2$ & Gaia DR2 1723454381704389248 \\
$106 \mathrm{c} 2$ & Gaia DR2 2248508292487691008 \\
$138 \mathrm{c} 2$ & Gaia DR2 2203366330791767552 \\
$140 \mathrm{c} 2$ & Gaia DR2 2005807146670408704 \\
$163 \mathrm{c} 2$ & Gaia DR2 537608345003955840 \\
$168 \mathrm{c} 2$ & Gaia DR2 534835788997551488 \\
$169 \mathrm{c} 2$ & Gaia DR2 533784174844087168 \\
$174 \mathrm{c} 2$ & Gaia DR2 2291000598671699200 \\
$184 \mathrm{c} 2$ & Gaia DR2 2206637339215351936 \\
$185 \mathrm{c} 2$ & Gaia DR2 2226453257466755328 \\
$191 \mathrm{c} 2$ & Gaia DR2 2231866565525667712 \\
G3 c2 & Gaia DR2 564698451789359104 \\
\hline
\end{tabular}

The main goal of this analysis is to identify all the members of the Cepheus association among our targets and to derive the maximum information about their physical and kinematic properties. This includes their multiplicity on both small (i.e., spectroscopic systems or visual binaries with small angular separation) and large (i.e., common proper-motion binaries) scales.

\section{B.1. Binary-pair candidates}

During our observing runs with the IDS spectrograph, it has happened that two sources are located at the coordinates of our targets. To be sure to observe the optical counterpart(s) of the unresolved X-ray source, we oriented the spectrograph slit with the position angle of such a binary-pair candidate to image them simultaneously. For the 18 targets listed in Table B.1 we acquired their long-slit spectra to characterize both of them. We subsequently determined if they are physically associated or if that is just a spurious association. We note that the sources \#6 and \#40 have two entries in this table. Since the separation between the two apertures was large enough to avoid any contamination of the spectrum extracted from the second aperture by the light of the bright source (and for the sake of homogenization of data reduction), we used the procedure of extraction within the IRAF
Table B.2. Spectroscopic systems among our targets.

\begin{tabular}{lll}
\hline \hline SB1 & 1RXS J043208.0+811627, & 1RXS J062558.2+822124, \\
& 1RXS J083407.4+791449, & 1RXS J093852.0+852625, \\
& 1RXS J193958.2+851032, & 1RXS J213749.2+803228, \\
& 1RXS J231616.5+784156, & 1RXS J232209.7+575626, \\
& 1RXS J232647.5+770304. & \\
\hline SB1? & 1RXS J155547.5+684014, & 1RXS J181048.9+701601, \\
& 1RXS J222706.6+652127. & \\
\hline SB2 & 1RXS J000142.0+773057, & 1RXS J000806.3+475659, \\
& 1RXS J003904.2+791912c1, & 1RXS J003941.9+790526, \\
& 1RXS J010929.0+683916, & 1RXS J011415.5+715933, \\
& 1RXS J011523.1+882923, & 1RXS J012927.4+744448, \\
& 1RXS J013925.5+701853c1, & 1RXS J023919.7+872828, \\
& 1RXS J024324.6+695320, & 1RXS J025538.5+544706, \\
& 1RXS J030926.6+673238, & 1RXS J040745.1+875030, \\
& 1RXS J061946.4+771933c2, & 1RXS J085353.7+870708, \\
& 1RXS J163747.2+723937, & 1RXS J170526.8+743600, \\
& 1RXS J171928.8+652227, & 1RXS J175910.1+584300, \\
& 1RXS J183627.4+715311, & 1RXS J193141.7+641951, \\
& 1RXS J195758.2+664253, & 1RXS J203857.5+580452, \\
& 1RXS J212929.0+621859, & 1RXS J214719.8+611618, \\
& 1RXS J224917.6+522634, & 1RXS 189583. \\
\hline Likely & 1RXS J005300.8+682125, & 1RXS J044912.7+773719, \\
SB2 & 1RXS J071743.1+764416, & 1RXS J181610.9+585539. \\
\hline SB2? & 1RXS J000002.5+733942c1, & 1RXS J010117.1+713114, \\
& 1RXS J045808.2+790813, & 1RXS J050642.4+745604c2, \\
& 1RXS J064241.4+880442, & 1RXS J075427.0+780633, \\
& 1RXS J161939.9+765515, & 1RXS J165315.4+701554, \\
& 1RXS J192127.4+611208, & 1RXS J195542.3+663207, \\
& 1RXS J203549.9+594930, & 1RXS J230822.7+790829, \\
\hline SB3 & 1RXS J235502.1+541516. & \\
\hline 1RXS J050642.4+745604c1, & 1RXS J185131.1+584258, \\
1RXS J232346.4+620620. & \\
\hline & 1RXS J211232.5+741227. & \\
\hline & &
\end{tabular}

Notes. Any source whose lithium line is visible in absorption is named in bold. With respect to the profile of the Ca I $\lambda 6717.7$ line, we also highlight those for which the Li I $\lambda 6707.8$ line is strong and very strong (see text for details).

environment, APALL, with the exception of the source \#40. Nevertheless, we applied the procedure detailed in Appendix A to estimate the average separation in pixels between the two apertures. We then converted it into an angular separation $\rho$ according to the spatial scale indicated above.

Based on our estimate of the angular separation and the position angle value recorded in the header of the raw data, we were able to identify each of sources located near one of our targets by relying mainly on the Gaia DR2 catalog (Table B.1).

\section{B.2. Comoving stars in Gaia DR2}

To search for any common proper-motion companions to our targets, we made use of a search cone of a radius of $2^{\prime}$ around each of them in Gaia DR2. Taking advantage of the very accurate astrometry in this catalog, we found comoving stars for 46 of our targets (Table 5). In $90 \%$ of cases, we identified one companion only. Nevertheless, the sources \#123, \#125, \#192, and F3 have two comoving companions, while we are able to list three for source \#149. These five targets have one close companion $\left(\rho<10^{\prime \prime}\right)$ and at least another more distant one $\left(\rho>40^{\prime \prime}\right)$.

For the closest systems ( $\pi \geq 4$ mas), we also extended the radius of search cone up to $10^{\prime}$. We then found that (i) the source \#6 has another common proper-motion companion with a large angular separation ( $\rho=3.44 \pm 0.185$ mas) and (ii) the sources G3 and G4 are separated by $4.96 \pm 0.033$ mas. 

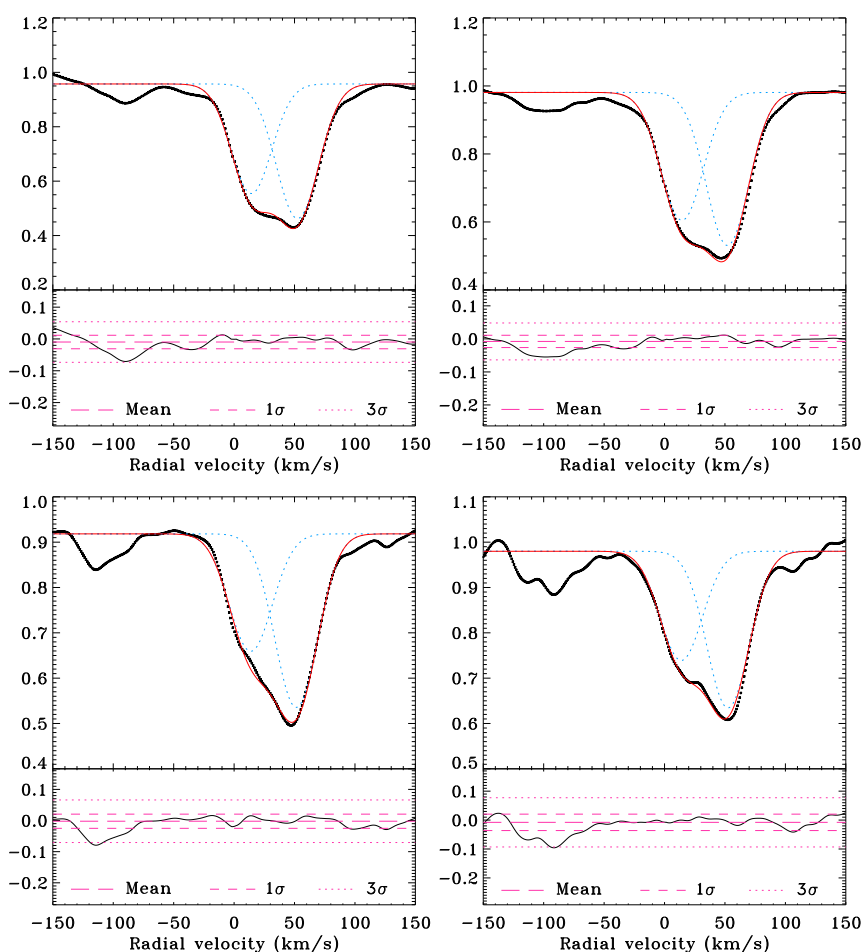

Fig. B.1. Cross-correlation function (asterisks) and Gaussian fitting (red solid line) for the source \#10 obtained from different orders of the SOPHIE spectrum (upper panel of each box). We denote the Gaussian fitting of each individual peak with the blue dotted lines. We also display the CCF residuals (lower panel of each box).

\section{B.3. Spectroscopic systems}

Table B.2 lists 44 spectroscopic systems found from our analysis of the CCF profiles (Sect. 3.1). We also include 17 possible spectroscopic systems for which the CCF profile is slightly asymmetric or the profile of the $\mathrm{H} \alpha$ line is double-peaked. In the latter case, the absence of a second peak in the CCF profile can be explained by a mass ratio of the two sources much different from 1, but that the activity of the fainter star is sufficiently strong so that its $\mathrm{H} \alpha$ line is visible in the spectrum obtained during the combined observation of the two sources.

Table B.2 also highlights the lithium-rich systems. Except for the SB1 systems, the determination of $W_{\mathrm{Li}}$ value for each component of the SB2 and SB3 systems goes beyond the scope of the work presented here (see Frasca et al. 2006; Klutsch et al. 2008 for a detailed analysis of SB2 and SB3 systems, respectively). We therefore decided to characterize the profile of the multiple Li I $\lambda 6707.8$ lines qualitatively. To this end, we compared their intensity with respect to that of the nearby $\mathrm{Ca}$ I $\lambda 6717.7$ line. We selected any system with Li I lines deeper than about the half of the CaI ones. We divided them into two groups by assuming that the $\mathrm{Li}$ I lines deeper than about the $\mathrm{Ca}$ I ones are very strong. The remaining sources are therefore listed as displaying a strong lithium line. Among the 44 spectroscopic systems, the lithium line is visible in the spectrum of $22(50 \%)$ sources. This includes nine $(20 \%)$ and six sources $(14 \%)$ with a strong and very strong lithium line, respectively.

Figure B.1 displays the complexity of some systems and the need to carefully analyze the CCF residues after subtracting the fitting, especially when the two peaks are severely blended (i.e., one peak with a clearly asymmetric shape) on several orders. We consider the source \#10 as an SB2 system observed near the conjunction but we have also to point out the possible detection of an additional smaller peak in its CCF profile. However its amplitude remains below our threshold.

\section{Appendix C: Low-mass stars}

We used Eq. (12) from Lépine et al. (2013) to convert the $V-J$ color index listed in the Table 1 into spectral type. We found that 45 of our targets are likely M-type stars (Table C.1). For six of them (\#20, \#48, \#54, \#61, \#62, \#69), we were not able to acquire a spectrum to confirm this classification.

Due to their low luminosity and our observational strategy, the survey of low-mass stars could only be conducted with the IDS spectrograph. For these sources, we normalized the spectra in two different ways to perform their spectral typing.

First, we defined the pseudo-continuum as a smooth line passing through the heads of molecular bands (see, e.g., Zapatero Osorio et al. 2002). In this way, all the molecular bands are fully in absorption and only the band-heads reach the 1.0 level. Such a task is impossible to be performed automatically. Normalized spectra are obtained by dividing each target spectrum by its continuum. This optimizes the comparison of our spectra with the Élodie library of standard stars, within the code ROTFIT. We refer the reader to Frasca et al. (2015) for an application of this code to M-type stars. The results are listed in Table 3.

Secondly, we divided each target spectrum by the continuum level in the spectral region near the $\mathrm{H} \alpha$ line. This procedure allows us to perform the spectral typing of the M-type stars through a comparative analysis of the spectral shape of our targets with those of standard stars observed with the same instrumental setup (Table E.1). To this end, we discarded the wavelength ranges contaminated by strong telluric lines or by chromospheric activity (e.g., the Balmer series). We looked for the best matches by means of a least-square minimization technique similar to that used by Klutsch et al. (2012) for the M-type stars from the CARMENES Input Catalog (CARMENCITA, Caballero et al. 2013). Based on an internal comparison of the CARMENES results as well as with three independent works, Alonso-Floriano et al. (2015) highlighted the reliability of this approach. The spectral typing resulting from both our procedures are fully compatible with each other. Table C.1 compares the spectral-type classifications resulting from our analysis with those available in the literature, and shows that they agree with each other with an accuracy of about one subclass. We finally found that three of these low-mass stars are late-K stars rather than early-M ones. Figure C. 1 shows the spectra of the 36 surveyed M-type stars, sorted by spectral type (from M0 to M4.5). 
Table C.1. Comparison of the spectral type derived for low-mass stars with those listed in the literature.

\begin{tabular}{|c|c|c|c|c|c|c|}
\hline \multirow[t]{2}{*}{$\#$} & \multicolumn{6}{|c|}{ Spectral type from: } \\
\hline & This work & Lépine et al. (2013) & CARMENCITA $^{(a)}$ & $\begin{array}{l}\text { SpT vs. } V-J \\
\text { relation }{ }^{(b)}\end{array}$ & Other(s) & Refs. \\
\hline 2 & $\mathrm{M} 3.5 \mathrm{~V}$ & M4V & & M3.4 & M3.5+M3.6, M4V & 9,11 \\
\hline 3 & $\mathrm{M} 2.5 \mathrm{~V}$ & & $\mathrm{M} 2.5 \mathrm{~V}$ & M2.4 & $\mathrm{M} 2.4 \mathrm{~V}$ & 8 \\
\hline 5 & M4V & M4V & $\mathrm{M} 4 \mathrm{~V}+\mathrm{M}$ & M3.6 & $\mathrm{M} 4.0+\mathrm{M} 4.5, \mathrm{M} 3.7 \mathrm{~V}$ & 7,8 \\
\hline $6 c 2$ & M1V & $\ldots$ & $\ldots$ & M0.4 & $\mathrm{M} 2 \mathrm{~V}$ & 6 \\
\hline 9 & M1V & $\mathrm{M} 1.5 \mathrm{~V}$ & $\ldots$ & M1.2 & $\ldots$ & $\ldots$ \\
\hline 12 & $\mathrm{M} 2.5 \mathrm{~V}$ & $\ldots$ & $\cdots$ & M0.9 & $\ldots$ & $\ldots$ \\
\hline 14 & $\mathrm{M} 3.5 \mathrm{~V}$ & $\ldots$ & $\ldots$ & M0.8 & $\ldots$ & $\ldots$ \\
\hline 15 & $\mathrm{M} 0.5 \mathrm{~V}$ & $\ldots$ & $\ldots$ & M3.1 & $\ldots$ & $\ldots$ \\
\hline 20 & & $\ldots$ & $\ldots$ & M2.4 & & \\
\hline 21 & M3V & $\ldots$ & $\ldots$ & M3.6 & M2e & 5 \\
\hline 23 & $\mathrm{M} 3.5 \mathrm{~V}$ & & & M1.9 & & \\
\hline 24 & $\mathrm{M} 1.5 \mathrm{~V}$ & $\mathrm{M} 2 \mathrm{~V}$ & M1V & M2.7 & M3 & 2 \\
\hline 26 & $\mathrm{M} 0.5 \mathrm{~V}$ & $\ldots$ & $\ldots$ & M2.5 & $\ldots$ & $\ldots$ \\
\hline 29 & MOV & $\ldots$ & $\ldots$ & M1.0 & $\ldots$ & $\ldots$ \\
\hline 30 & M4V & $\ldots$ & $\ldots$ & M3.7 & $\ldots$ & $\ldots$ \\
\hline 34 & M1V & $\ldots$ & $\ldots$ & M1.8 & $\ldots$ & $\ldots$ \\
\hline 35 & $\mathrm{M} 0.5 \mathrm{~V}$ & $\ldots$ & $\ldots$ & M1.3 & & $\ldots$ \\
\hline 38 & M0V & $\ldots$ & $\ldots$ & K7.85 & K5/M0 & 3 \\
\hline 39 & M3.5V & & & M3.8 & & \\
\hline 48 & $\ldots$ & M4V & $\mathrm{M} 3.5 \mathrm{~V}$ & M4.1 & $\mathrm{M} 4 / 5$ & 10 \\
\hline 54 & $\ldots$ & $\ldots$ & $\ldots$ & M2.8 & $\mathrm{M} 3 / 4$ & 10 \\
\hline 61 & $\ldots$ & & $\ldots$ & M1.0 & $\ldots$ & $\ldots$ \\
\hline 62 & $\ldots$ & M4V & $\ldots$ & M4.1 & $\ldots$ & $\ldots$ \\
\hline 69 & & & $\ldots$ & M2.4 & $\ldots$ & $\ldots$ \\
\hline 70 & M3V & $\mathrm{M} 2.5 \mathrm{~V}$ & $\ldots$ & M1.7 & $\ldots$ & $\ldots$ \\
\hline 73 & M1V & $\ldots$ & $\ldots$ & M1.4 & $\ldots$ & $\ldots$ \\
\hline 80 & $\mathrm{M} 1.5 \mathrm{~V}$ & $\ldots$ & $\ldots$ & M1.9 & $\ldots$ & $\ldots$ \\
\hline $81 \mathrm{c} 1$ & M1V & $\begin{array}{l}\cdots \\
\cdots\end{array}$ & $\begin{array}{l}\cdots \\
\cdots\end{array}$ & $\ldots$ & $\begin{array}{l}\cdots \\
\cdots\end{array}$ & $\begin{array}{l}\cdots \\
\cdots\end{array}$ \\
\hline $81 \mathrm{c} 2$ & $\mathrm{M} 2.5 \mathrm{~V}$ & $\ldots$ & $\ldots$ & & $\ldots$ & \\
\hline 85 & M2V & $\ldots$ & & M1.2 & $\mathrm{M} 2 \mathrm{~V}$ & 11 \\
\hline 88 & M4V & M4V & M3.5V & M3.6 & M4Ve & 4 \\
\hline 107 & MOV & $\ldots$ & $\ldots$ & M0.5 & $\ldots$ & $\ldots$ \\
\hline 111 & M1V & $\cdots$ & $\begin{array}{l}\cdots \\
\cdots\end{array}$ & M1.4 & $\begin{array}{l}\cdots \\
\cdots\end{array}$ & $\cdots$ \\
\hline 113 & M0V & MOV & $\ldots$ & M0.6 & $\ldots$ & $\ldots$ \\
\hline 121 & $\mathrm{M} 3.5 \mathrm{~V}$ & $\ldots$ & $\ldots$ & M4.6 & $\ldots$ & $\ldots$ \\
\hline 124 & K7V & $\ldots$ & $\ldots$ & M0.2 & $\ldots$ & $\ldots$ \\
\hline 136 & K6V & $\ldots$ & $\ldots$ & M0.2 & $\ldots$ & $\ldots$ \\
\hline 143 & MOV & $\ldots$ & $\ldots$ & M2.0 & $\ldots$ & $\ldots$ \\
\hline 146 & $\mathrm{M} 0.5 \mathrm{~V}$ & $\ldots$ & & M2.6 & $\ldots$ & \\
\hline 151 & MOV & $\mathrm{M} 0.5 \mathrm{~V}$ & MOV & M1.0 & M0V, M0.3V & 1,8 \\
\hline 154 & $\mathrm{M} 0.5 \mathrm{~V}$ & $\ldots$ & $\ldots$ & M1.9 & $\ldots$ & $\ldots$ \\
\hline 161 & $\mathrm{M} 1.5 \mathrm{~V}$ & $\ldots$ & $\ldots$ & M2.6 & $\ldots$ & $\ldots$ \\
\hline 189 & K7V & $\ldots$ & $\ldots$ & M1.1 & $\ldots$ & $\ldots$ \\
\hline $191 \mathrm{c} 1$ & MOV & $\ldots$ & $\ldots$ & M1.2 & K7V & 6 \\
\hline $191 \mathrm{c} 2$ & M2V & $\ldots$ & $\ldots$ & $\ldots$ & M3V & 6 \\
\hline
\end{tabular}

Notes. ${ }^{(a)}$ CARMENCITA stands for "CARMENes Cool star Information and daTa Archive" and is a private database of the CARMENES consortium (http://carmenes. caha.es/). ${ }^{(b)}$ We converted the $V-J$ color index into spectral type by using Eq. (12) of Lépine et al. (2013).

References. $1=$ Vyssotsky (1956); $2=$ Lee (1984); 3 = Stephenson (1986); $4=$ Fleming et al. (1988); $5=$ Motch et al. (1998); $6=$ Tachihara et al. (2005); 7 = Daemgen et al. (2007); 8 = Shkolnik et al. (2009); 9 = Shkolnik et al. (2010); 10 = Gigoyan \& Mickaelian (2012); and 11 = Janson et al. (2012). 
A. Klutsch et al.: Properties of the young stellar association in Cepheus
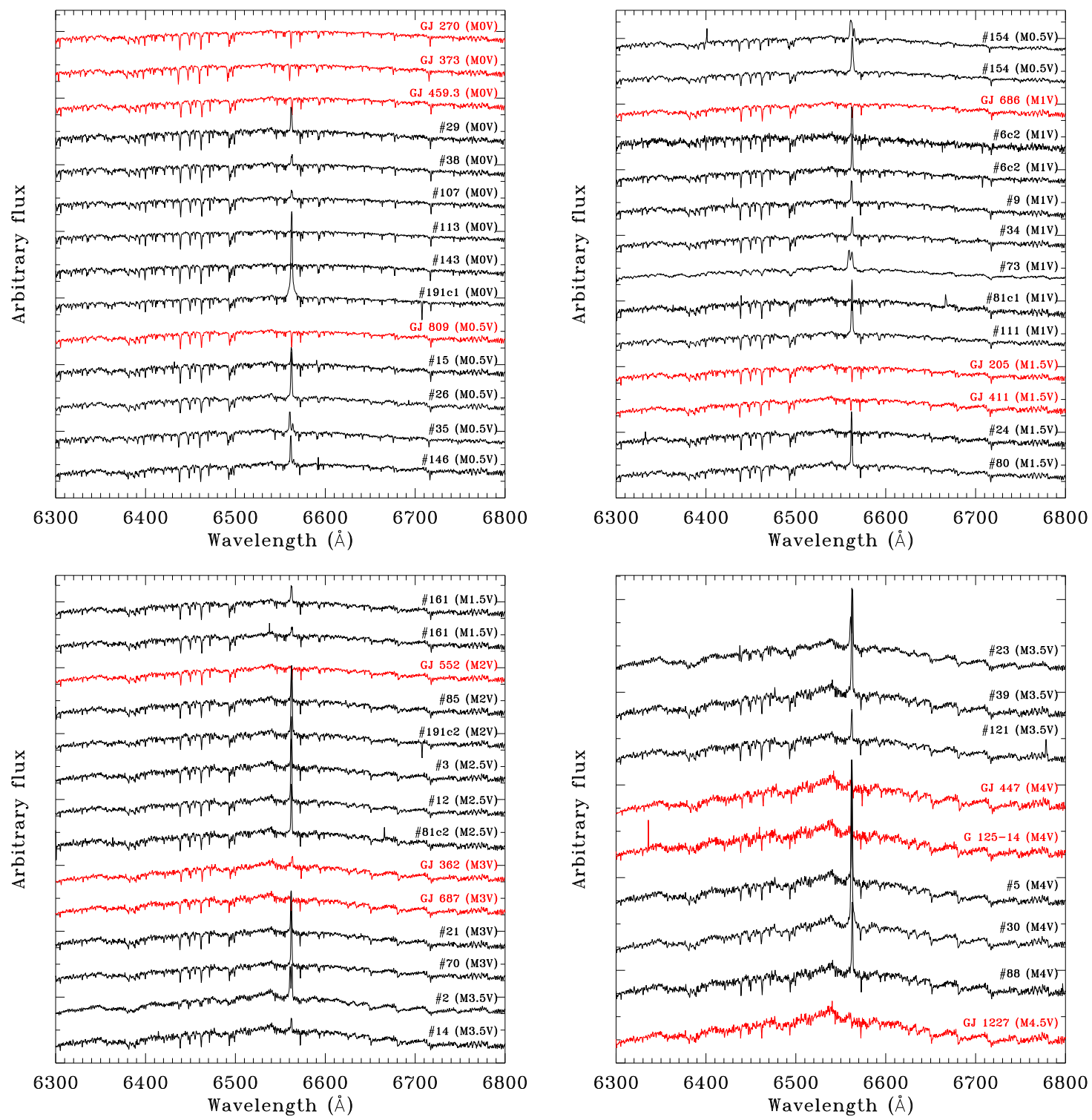

Fig. C.1. Sequential distribution of spectra for the M-type stars. Their coloring clearly distinguishes targets (black) from standards (red).

\section{Appendix D: Information about some stars}

1RXS J003904.2+791912. (Source \#6): The infrared counterpart identified for this source corresponds to the faint companion of the source [TNK2005] 5 that is known as a WTTS in Cepheus (Tachihara et al. 2005). Our target was only detected in photometry and classified as an M2-type companion candidate by the aforementioned authors. During one of our observing nights, both components were positioned simultaneously on the slit. These display a deep lithium absorption and the brightest component is an SB2 system (Fig. D.1). Based on our analysis, this source therefore is a hierarchical triple system composed of a close inner binary plus a tertiary component in a long-period orbit. Moreover, we found an additional wide-separation companion $(\rho=3.44 \pm 0.185$ mas; see Table 5). All these stars are associated to Cep I.

1RXS J010112.8+570839. (Source \#8): While its intrinsic color agrees with a late-M giant (see Fig. 4, along with Fig. 2 of Jiménez-Esteban et al. 2012), we classified it as a K5 giant with a large uncertainty on its surface gravity $(\log g=$ $4.20 \pm 0.95 \mathrm{dex})$. This is consistent with the extinction of $A_{V} \sim$

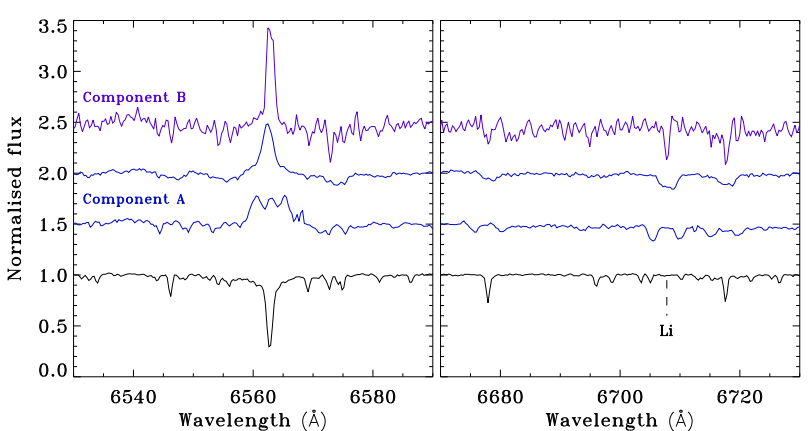

Fig. D.1. H $\alpha$ and lithium spectra of TYC 4500-1549-1 (\#6) found as a visual binary. Two of spectra for the bright component are also shown.

0.94 mag derived from our SED analysis (Sect. 3.3). Moreover, this source is more than 2 mag fainter compared to giants included in the LSPM-North catalog (Lépine \& Shara 2005), with a similar $J-K_{S}$ color index.

$1 R X S J 174104.6+842458$. (Source \#81): This visual binary is composed of an M1 and an M2.5-type star, with a small angu- 

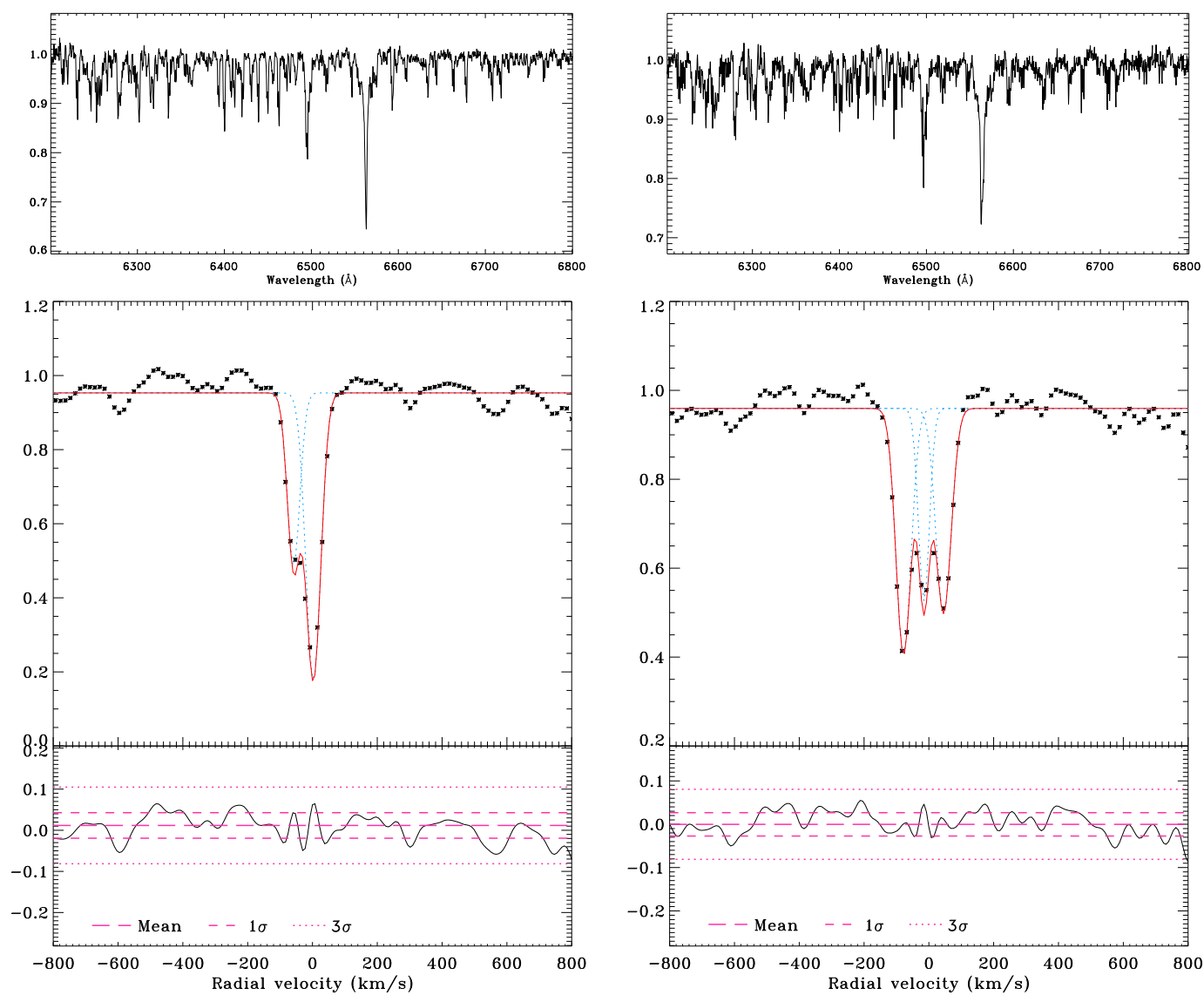

Fig. D.2. IDS spectra of the source $\# 92$ obtained in a time span of about $24 \mathrm{~h}$ (top panels) and their associated CCF profiles (bottom panels).

lar separation (Table 5). These are the only M-type stars displaying the He I $\lambda 6678 \AA$ line in emission (Fig. C.1).

$1 R X S J 185131.1+584258$. (Source \#92): We observed this source thrice. At the first epoch, it appears as an SB3 system. We then re-observed this star during our IDS runs in order to learn more about the temporal variation of this system. Since the source turned out to be an SB2 system, we repeated the observation. Within this time span of about one day its spectrum has drastically changed and a third peaks is clearly visible in the $\mathrm{CCF}$ profile (Fig. D.2). At this stage, it is complicated to put forward any hypothesis about the dynamics of this system, but the radial velocity of the three components seem to change quickly. This is in contrast with the three SB3 systems analyzed by Klutsch et al. (2008).

1RXS J192215.6+673918. (Source \#99): We selected the source 2MASS J19221478+6739142 as its infrared counterpart. With an angular separation of $\sim 6.42$, this source fulfilled all our criteria. Its infrared colors suggest a late M-type star according to West et al. (2008). At this position, however, we observed a giant star $\left(T_{\mathrm{eff}}=4835 \pm 102 \mathrm{~K}, \log g=2.80 \pm 0.23 \mathrm{dex}\right.$, and spectral type $=$ G9 III) with a small lithium absorption line: $\mathrm{EW}(\mathrm{Li})=16 \pm 11 \mathrm{~m} \AA$, and $\log \mathrm{N}(\mathrm{Li}) \sim 0.9$ dex. Seeking to understand the inconsistency between the photometric and spectroscopic data, we found that 2MASX 19221485+6739143 from the catalog of the 2MASS extended sources (Skrutskie et al. 2003) has similar coordinates. Therefore the infrared counterpart of this target is indeed the galaxy GALEX J192214.8+673914.

It appears that we observed the source TYC 4444-1831-1 (or 2MASS J19221289+6739053) that is somewhat more distant with a separation of $\sim 20$ '42 to the X-ray source. We illustrated

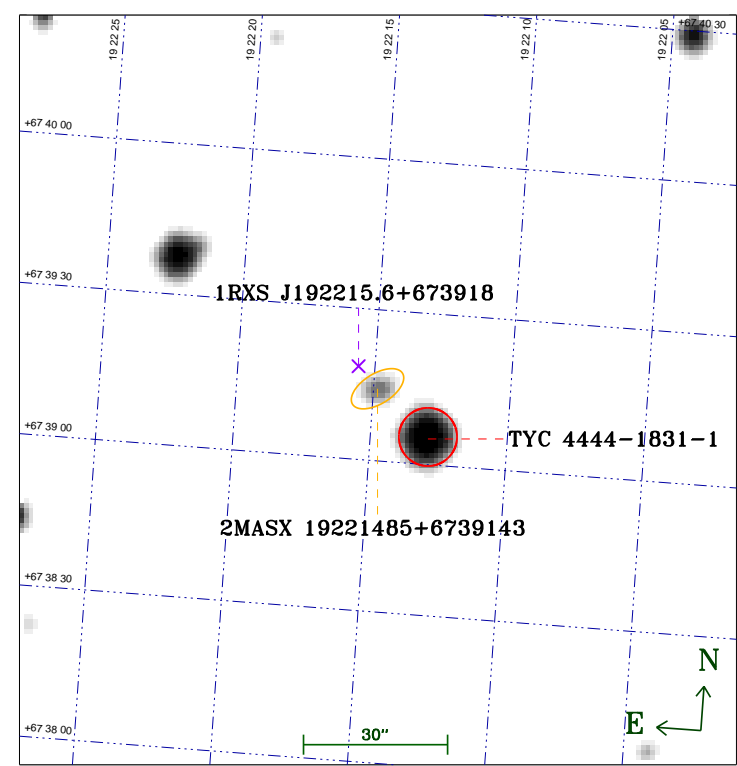

Fig. D.3. Photographic $B_{J}$-band image of the second Palomar Observatory Sky Survey (POSS-II, epoch J1992.57), centered on the 2MASS extended source 2MASX 19221485+6739143. We also show the positions of the X-ray source 1RXS J192215.6+673918 (\#99) and the optical source TYC 4444-1831-1.

the space configuration of these three sources on Fig. D.3. Moreover, its photometry is consistent with a late- $\mathrm{G}$ or early-K star and its small proper motions are in agreement with the expectation for a giant star. The probabilities of Flesch (2010) are rather 


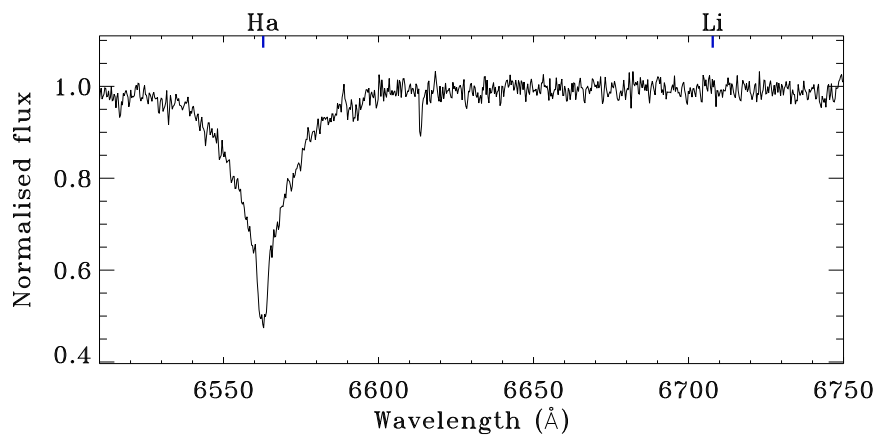

Fig. D.4. IDS spectrum of source \#139.

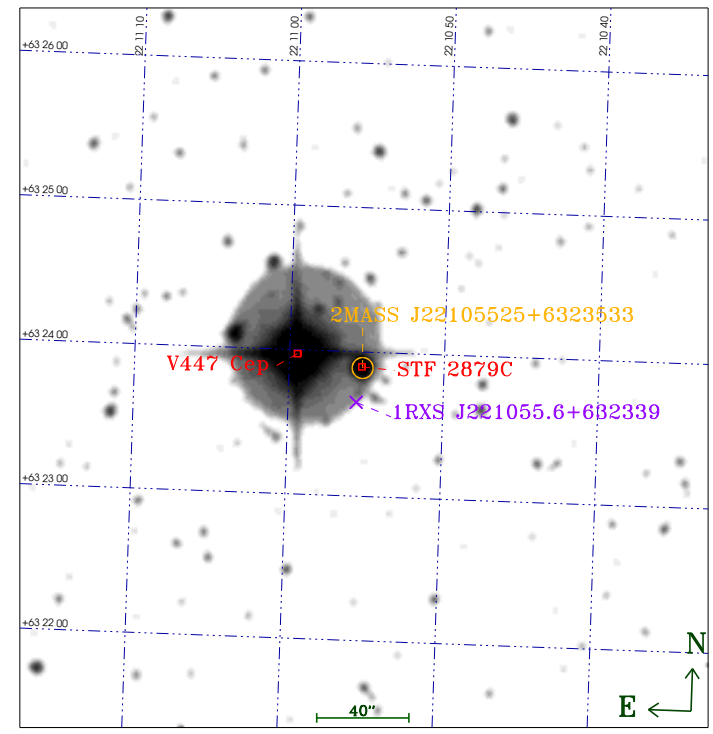

Fig. D.5. Photographic $B_{J}$-band image of the second Palomar Observatory Sky Survey (POSS-II), centered on the infrared source 2MASS J22105525+6323533. We mark the locus of the X-ray source 1RXS J221055.6+632339 (\#139) and its possible optical counterpart STF 2879 C, which is the companion of the bright B5 binary V447 Cep.

close that the radio/X-ray association is erroneous (51\%) and this $\mathrm{X}$-ray source is a star (42\%), while the probability to be a galaxy is only of $7 \%$.

1RXS J221055.6+632339. (Source \#139): As in Motch et al. (1997b), we consider the early-type star STF 2879 C (Fig. D.4) as being its optical counterpart due to its $B-V$ color index of about $0.75 \pm 0.44 \mathrm{mag}$ from the GSC II photometric data. In contrast, the APASS catalog reported a more reliable color index $(B-V=0.320 \pm 0.031 \mathrm{mag})$. This source has probably passed through the cracks because of its proximity to the B5 binary V447 Cep whose extended halo of light could affect the entire photometry of our target (Fig. D.5). Nevertheless, the particularity of this source is not limited to this aspect. Its magnitudes quoted in the final release of the WISE all-sky survey catalog are also atypical. With a $W 1$ magnitude of $10.819 \pm 0.023 \mathrm{mag}$ and a $W 1-W 4$ of $4.691 \pm 0.050 \mathrm{mag}$, this source falls in the area mainly populated by T Tauri stars displaying an infrared excess (Guillout, priv. comm.).

1RXS 184257. (Source \#160): This source (=[KP93] 2-43 in Kun \& Prusti 1993) is too faint in optical to acquire a spectrum with the instruments at our disposal in a reasonable exposure time. It is known as a visual binary whose primary component is a young star (1 Myr-old WTTS in Kun et al. 2009 or a CTTS in Simon 2009) and one of the strongest X-ray emitters during the XMM-Newton and Chandra observations of the L1251 cloud (Simon 2006, 2009).

1RXS J003941.9+790526. (Source G4): We followed a procedure similar to Frasca et al. (2006) to derive its orbital parameters. For this purpose we combined the radial velocities that we previously reported in Paper III with those obtained during the observing runs analyzed in the current paper. However it turns out that most measurements (HJD $=2455077-2455111$ ) only cover half of the orbit of this system. From our preliminary analysis the period would be estimated at $\sim 70$ days.

\section{Appendix E: Additional materials online}
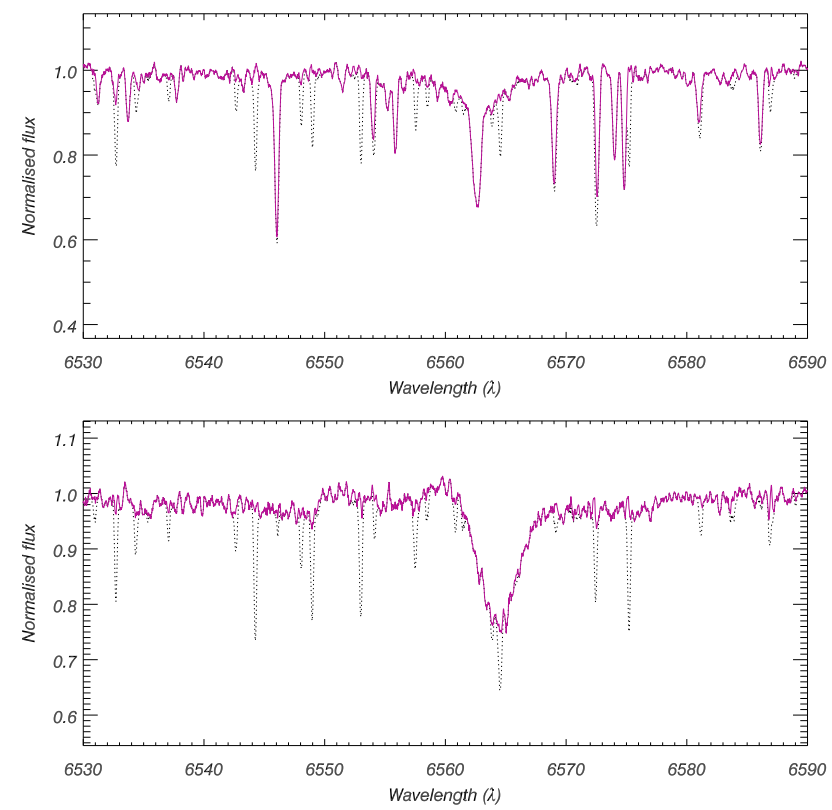

Fig. E.1. Results of our telluric line removal on SOPHIE spectra for a slow-rotating source (top panel) and a fast rotator (bottom panel). On each panel, the black dotted lines and purple solid lines show the spectra before and after the application of our procedure, respectively.

All long online tables are available at the CDS. Table 1 summarizes the optical and infrared names of each X-ray source, along with those appearing in Simbad and some main parameters coming from the literature. The three additional online tables present our results. Table 3 reports the radial velocity of all the stars, including the measurements for each component of a spectroscopic system, along with the rotational velocity, atmospheric parameters, and lithium equivalent widths derived for the targets identified as single stars or SB1 systems. Table 4 provides the astrometry in Gaia DR2, the Galactic positions, and the extinction estimate of our targets, as well as the space velocities of those identified as single stars. Table 5 lists all the sources that are comoving with one of our targets.

To derive the radial and rotational velocities of our targets and to perform their spectral typing, we made use of a smaller library of template spectra taken with FOCES and IDS during our observing runs. Their spectral type, atmospheric parameters, and radial and rotational velocities are given in Table E.1.

We show the efficiency of our procedure for removing telluric lines in the case of a few SOPHIE spectra (Fig. E.1). For each target observed during our survey, we display only one spectrum in the region around the Li I $\lambda 6707.8$ line (Fig. E.2). 
Table E.1. Basic parameters of reference stars observed during our runs.

\begin{tabular}{|c|c|c|c|c|c|c|c|c|c|c|}
\hline Star name & $\mathrm{SpT}^{(a)}$ & Ref. & $\begin{array}{c}T_{\text {eff }}{ }^{(b)} \\
(\mathrm{K})\end{array}$ & $\begin{array}{c}\log g^{(b)} \\
\left(\mathrm{cm}^{2} \mathrm{~s}^{-1}\right)\end{array}$ & $\begin{array}{r}{[\mathrm{Fe} / \mathrm{H}]^{(b)}} \\
(\mathrm{dex})\end{array}$ & Ref. & $\begin{array}{l}v \sin i^{(c)} \\
\left(\mathrm{km} \mathrm{s}^{-1}\right)\end{array}$ & Ref. & $\begin{array}{c}\langle R V\rangle^{(d)} \\
\left(\mathrm{km} \mathrm{s}^{-1}\right)\end{array}$ & $o_{\mathrm{RV}}$ \\
\hline HD 187691 & F8 V & A3 & 6173 & 4.25 & 0.04 & B15 & 3.6 & $\mathrm{C} 3$ & 0.03 & 6 \\
\hline HD 19373 & F9.5 V & A4 & 6008 & 4.33 & 0.15 & B12 & 4.5 & B11 & 49.4 & 1 \\
\hline HD 22879 & G0 V & A3 & 5759 & 4.25 & -0.85 & B14 & 2.3 & $\mathrm{C} 3$ & 120.31 & 4 \\
\hline HD 10307 & G1 V & A4 & 5859 & 4.27 & 0.04 & B15 & 2.3 & $\mathrm{C} 3$ & 4.97 & 10 \\
\hline HD 159222 & G1 V & A4 & 5851 & 4.41 & 0.16 & B12 & 3.3 & B11 & -51.62 & 15 \\
\hline HD 196850 & G1 V & A4 & 5838 & 4.37 & -0.09 & B16 & 2.0 & В02 & -21.07 & 9 \\
\hline HD 197076 & G1 V & A4 & 5828 & 4.45 & -0.10 & B15 & 2.7 & $\mathrm{C} 7$ & -35.44 & 13 \\
\hline HD 19445 & $\mathrm{G} 2 \mathrm{~V} \mathrm{Fe}-3$ & A4 & 5918 & 4.41 & -1.89 & B08 & 4.1 & $\mathrm{C} 2$ & -139.84 & 2 \\
\hline HD 193664 & G3 V & A5 & 5886 & 4.48 & -0.09 & B02 & 1.3 & B02 & -4.51 & 28 \\
\hline HD 65583 & G8 V & $\mathrm{A} 1$ & 5279 & 4.76 & -0.69 & B02 & 3.3 & $\mathrm{C} 8$ & $14.89^{(3)}$ & 20 \\
\hline HD 10700 & $\mathrm{G} 8.5 \mathrm{~V}$ & A6 & 5290 & 4.46 & -0.48 & B11 & 0.9 & C3 & -16.65 & 1 \\
\hline HD 10780 & G9 V & $\mathrm{A} 4$ & 5309 & 4.56 & 0.00 & B04 & 1.3 & B01 & 2.72 & 33 \\
\hline HD 12051 & G9 V & A4 & 5458 & 4.55 & 0.24 & B07 & 0.5 & B06 & -35.22 & 4 \\
\hline HD 185144 & G9 V & A4 & 5204 & 4.37 & -0.26 & B15 & 0.8 & $\mathrm{C} 3$ & 26.61 & 1 \\
\hline HD 182488 & $\mathrm{G} 9+\mathrm{V}$ & A4 & 5393 & 4.55 & 0.22 & B05 & 1.2 & $\mathrm{C} 3$ & -21.57 & 12 \\
\hline HD 3651 & K0 V & A4 & 5218 & 4.52 & 0.20 & B05 & 1.2 & $\mathrm{C} 2$ & -33.08 & 2 \\
\hline HD 38230 & K0 V & A4 & 5174 & 4.53 & -0.08 & B02 & 0.9 & $\mathrm{C} 3$ & -29.22 & 33 \\
\hline HD 10476 & $\mathrm{~K} 1 \mathrm{~V}$ & A6 & 5173 & 4.59 & -0.08 & B04 & 1.7 & B01 & -33.76 & 3 \\
\hline HD 4628 & $\mathrm{~K} 2 \mathrm{~V}$ & A4 & 4905 & 4.60 & -0.36 & B07 & 1.5 & B06 & -10.35 & 1 \\
\hline HD 73667 & $\mathrm{~K} 2 \mathrm{~V}$ & A4 & 4884 & 4.40 & -0.58 & B07 & 1.2 & B01 & -12.12 & 21 \\
\hline HD 166620 & $\mathrm{~K} 2 \mathrm{~V}$ & A4 & 5007 & 4.62 & -0.24 & B04 & 0.6 & $\mathrm{C} 3$ & -19.59 & 3 \\
\hline HD 16160 & K3 V & A6 & 4829 & 4.60 & -0.16 & B07 & 0.9 & B06 & 25.73 & 1 \\
\hline HD 219134 & K3 V & A4 & 4835 & 4.56 & 0.12 & B02 & 1.8 & B01 & -18.69 & 4 \\
\hline HD 190007 & $\mathrm{~K} 4 \mathrm{~V}$ & A4 & 4786 & 4.31 & -0.02 & B10 & 0.9 & B10 & -30.43 & 10 \\
\hline HD 201091 & K5 V & A4 & 4236 & 4.50 & -0.03 & B07 & 3.8 & B06 & -65.51 & 5 \\
\hline HD 221503 & K6 V & A6 & 4270 & 4.99 & 0.02 & B13 & 2.5 & $\mathrm{C} 4$ & $-0.96^{(5)}$ & 6 \\
\hline HD 201092 & K7 V & A4 & 4200 & 4.60 & -0.63 & B09 & 1.6 & $\mathrm{C} 3$ & -64.15 & 5 \\
\hline GJ 270 & M0 V & A9 & 3856 & 4.69 & $\ldots$ & B17 & $\leq 3.0$ & C9 & $-69.78^{(4)}$ & 1 \\
\hline GJ 373 & M0 V & A9 & 3820 & 4.68 & $\ldots$ & B17 & $\leq 2.0$ & $\mathrm{C} 9$ & $15.13^{(4)}$ & 4 \\
\hline GJ 459.3 & M0V & A9 & 3852 & 4.66 & $\ldots$ & B17 & $\leq 3.0$ & C9 & -0.64 & 1 \\
\hline GJ 809 & M0.5 V & A9 & 3720 & 4.67 & -0.13 & B01 & $\leq 2.0$ & C9 & -17.55 & 10 \\
\hline GJ 686 & M1 V & A9 & 3611 & 4.84 & -0.44 & A8 & $\leq 2.0$ & C9 & -9.87 & 11 \\
\hline GJ 205 & $\mathrm{M} 1.5 \mathrm{~V}$ & A9 & 3626 & 4.80 & 0.60 & B09 & $\leq 2.0$ & C9 & 8.25 & 15 \\
\hline GJ 411 & $\mathrm{M} 1.5 \mathrm{~V}$ & A9 & 3671 & 4.89 & $\ldots$ & B17 & $\leq 2.0$ & C9 & $-84.71^{(4)}$ & 7 \\
\hline GJ 552 & M2 V & A9 & 3574 & 4.79 & $\ldots$ & B17 & $\leq 2.0$ & C9 & $7.36^{(4)}$ & 2 \\
\hline GJ 362 & M3 V & A9 & 3430 & 4.85 & $\ldots$ & B17 & $\leq 2.0$ & C9 & 6.21 & 2 \\
\hline GJ 687 & M3 V & A9 & 3340 & 4.82 & 0.15 & B01 & $\leq 2.5$ & C9 & -29.16 & 9 \\
\hline GJ 447 & M4 V & A9 & 3192 & 5.07 & $\ldots$ & B17 & $\leq 2.0$ & C9 & $-30.86^{(4)}$ & 1 \\
\hline GJ 1227 & M4.5 V & A8 & 3072 & 5.01 & $\ldots$ & B17 & $\leq 2.0$ & C9 & -14.04 & 6 \\
\hline HD 84737 & G0 IV-V & $\mathrm{A} 4$ & 5934 & 4.16 & 0.16 & B12 & 3.0 & B11 & $4.79^{(1)}$ & 25 \\
\hline HD 51000 & G5 III & A2 & 5180 & 3.05 & -0.04 & B03 & 2.3 & В03 & -9.36 & 1 \\
\hline HD 62509 & G9 III & A7 & 4955 & 3.07 & 0.16 & B15 & 2.4 & $\mathrm{C} 3$ & 3.24 & 2 \\
\hline HD 124897 & K0 III & A4 & 4280 & 1.69 & -0.52 & B06 & 2.5 & $\mathrm{C} 3$ & -5.30 & 8 \\
\hline HD 12929 & K1 III & A4 & 4546 & 2.40 & -0.24 & B04 & 1.6 & $\mathrm{C} 3$ & $-14.64^{(2)}$ & 170 \\
\hline HD 26162 & K2 III & $\mathrm{A} 2$ & 4800 & 2.90 & 0.06 & B03 & 4.0 & B03 & 24.74 & 7 \\
\hline HD 29139 & K5 III & A6 & 3891 & 1.20 & -0.15 & B06 & 2.6 & $\mathrm{C} 3$ & 54.02 & 8 \\
\hline
\end{tabular}

Notes. We adopted the spectral type, atmospheric parameters, and projected rotational velocities found in the literature (see references below). We also determined the mean $R V$ values from those listed in the online Élodie archive, along with the number of observations used $o_{\mathrm{RV}}$.

References. ${ }^{(a)}$ For the G- and K-type sources, we list the spectral type most often cited in the last release of the catalog of stellar spectral classification (Skiff 2010): A1 = Harlan (1969), A2 = Cowley \& Bidelman (1979), A3 = Gray et al. (2001), A4= Gray et al. (2003), A5 = Abt \& Willmarth (2006), A6 = Gray et al. (2006), A7 = Abt (2008), and A8 = Jenkins et al. (2009). For the M-type stars, we usually adopt the spectral type from Alonso-Floriano et al. (2015), labeled A9. ${ }^{(b)}$ The atmospheric parameters come from: B01 =Woolf \& Wallerstein (2005), B02 = Valenti \& Fischer (2005), B03 = Hekker \& Meléndez (2007), B04 = Ramírez et al. (2007), B05=Fuhrmann (2008), B06 = Meléndez et al. (2008), B07 = Mishenina et al. (2008), B08 = Reddy \& Lambert (2008), B09 = Frasca et al. (2009), B10= Guillout et al. (2009), B11 = Bruntt et al. (2010), B12= Gonzalez et al. (2010), B13=Casagrande et al. (2010), B14= Nissen \& Schuster (2010), B15 = da Silva et al. (2011), B16= Casagrande et al. (2011), and B17 = Stassun et al. (2018). Most of them are available in the PASTEL catalog (Soubiran et al. 2010). ${ }^{(c)} v \sin i$ values from: C1 = Delfosse et al. (1998), C2 = Fischer $\&$ Valenti (2005), C3= the catalog of stellar rotational velocities of Glebocki \& Gnacinski (2005), C4 = Torres et al. (2006), C5 = Houdebine (2010), C6 = Houdebine (2011), C7 = Herrero et al. (2012), C8 = McCarthy \& Wilhelm (2014), and C9 = Jeffers et al. (2018). Some of these publications contain also an AP determination. ${ }^{(d)}$ We also made use of the $R V$ values from ${ }^{(1)}$ Soubiran et al. (1998), (2) Famaey et al. (2005), ${ }^{(3)}$ Soubiran et al. (2013), (4) Jeffers et al. (2018), and ${ }^{(5)}$ Soubiran et al. (2018). 
A. Klutsch et al.: Properties of the young stellar association in Cepheus
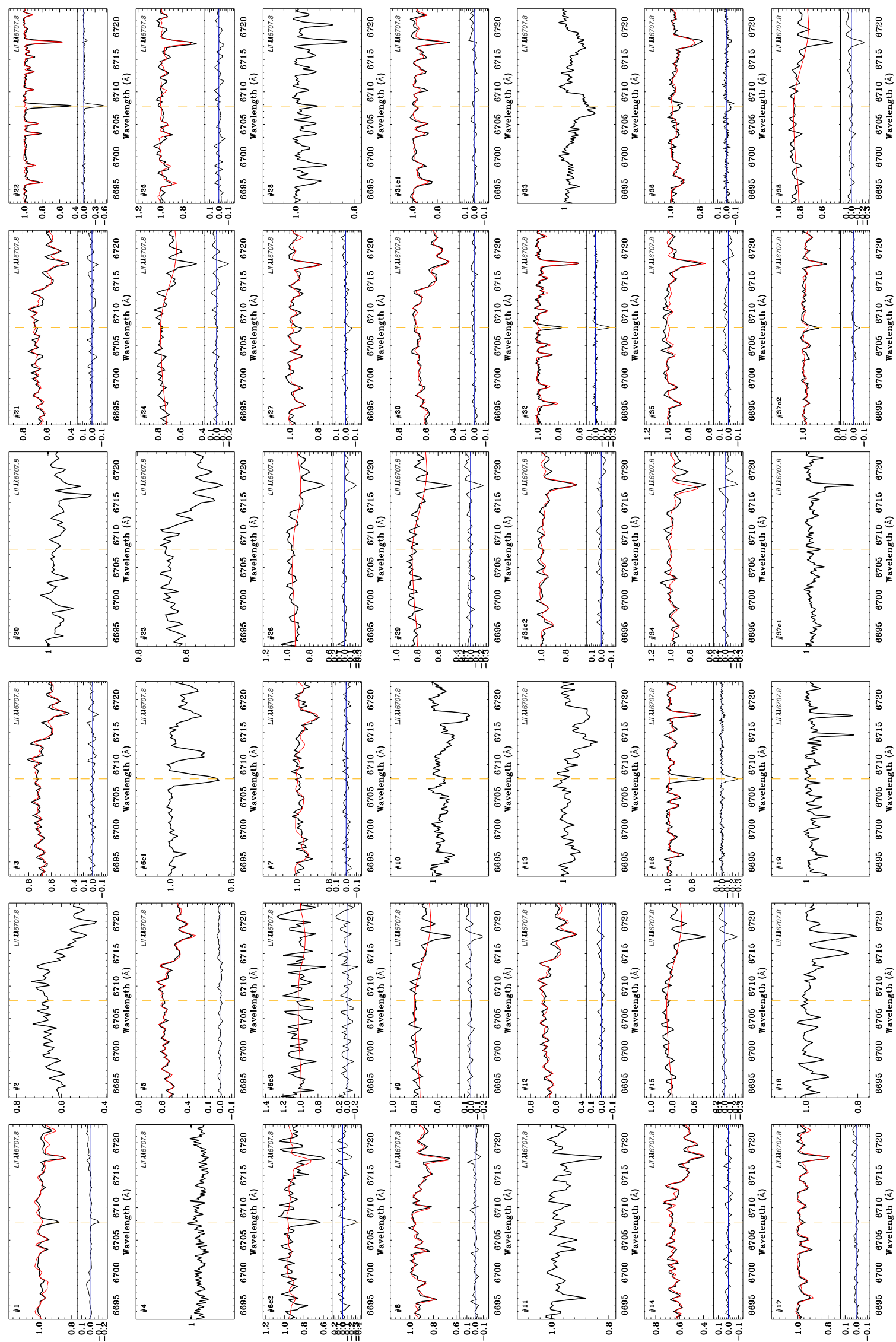

Fig. E.2. Target spectra (black line) around the lithium line (vertical dashed line). We display the best match rotationally broadened to the $v$ sin $i$ obtained with ROTFIT with the red line only for single stars and SB1 systems (upper panel), while the difference (target-template) spectrum shows their lithium content (lower panel). The number of the source appears in the upper left corner. 

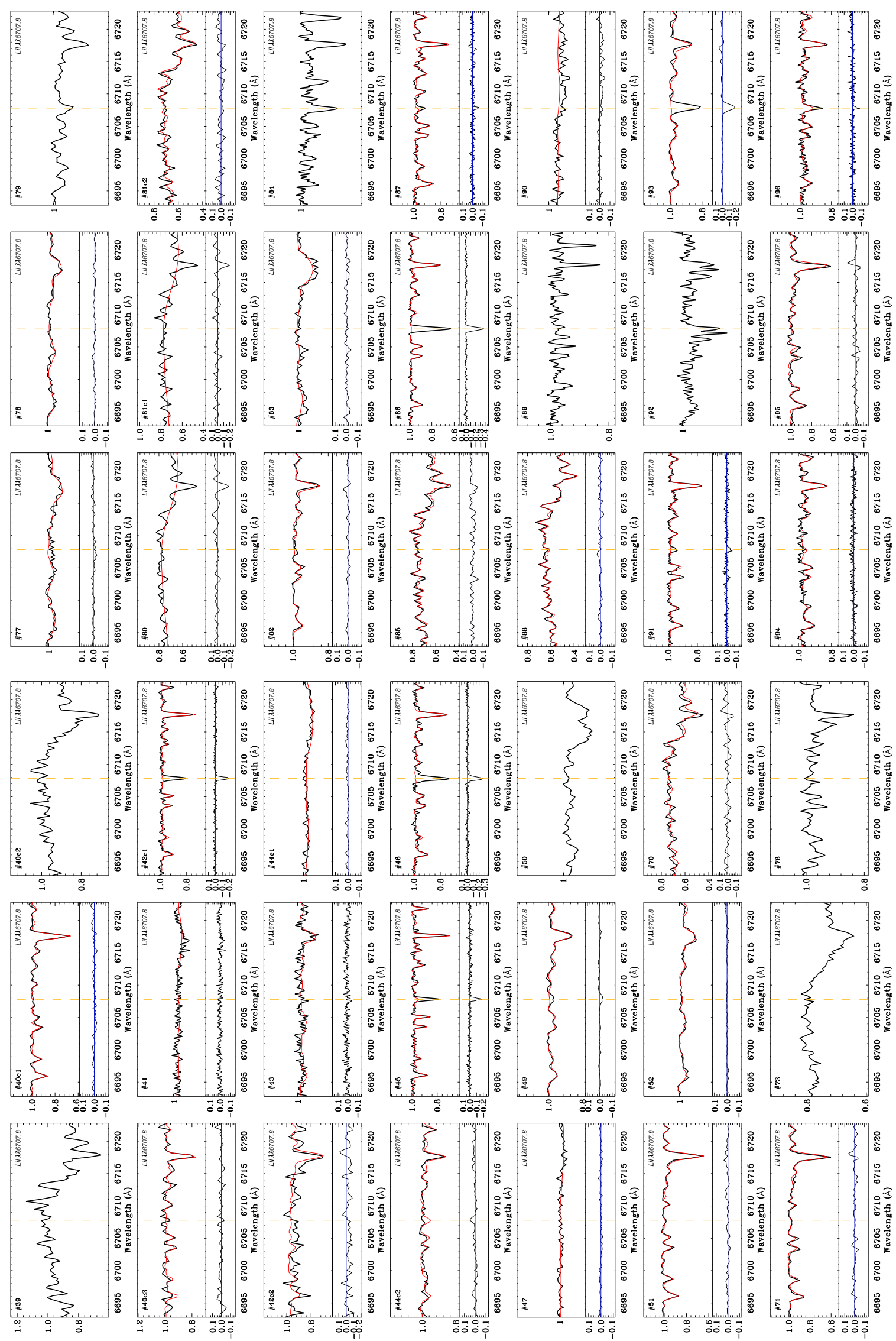

Fig. E.2. continued. 
A. Klutsch et al.: Properties of the young stellar association in Cepheus
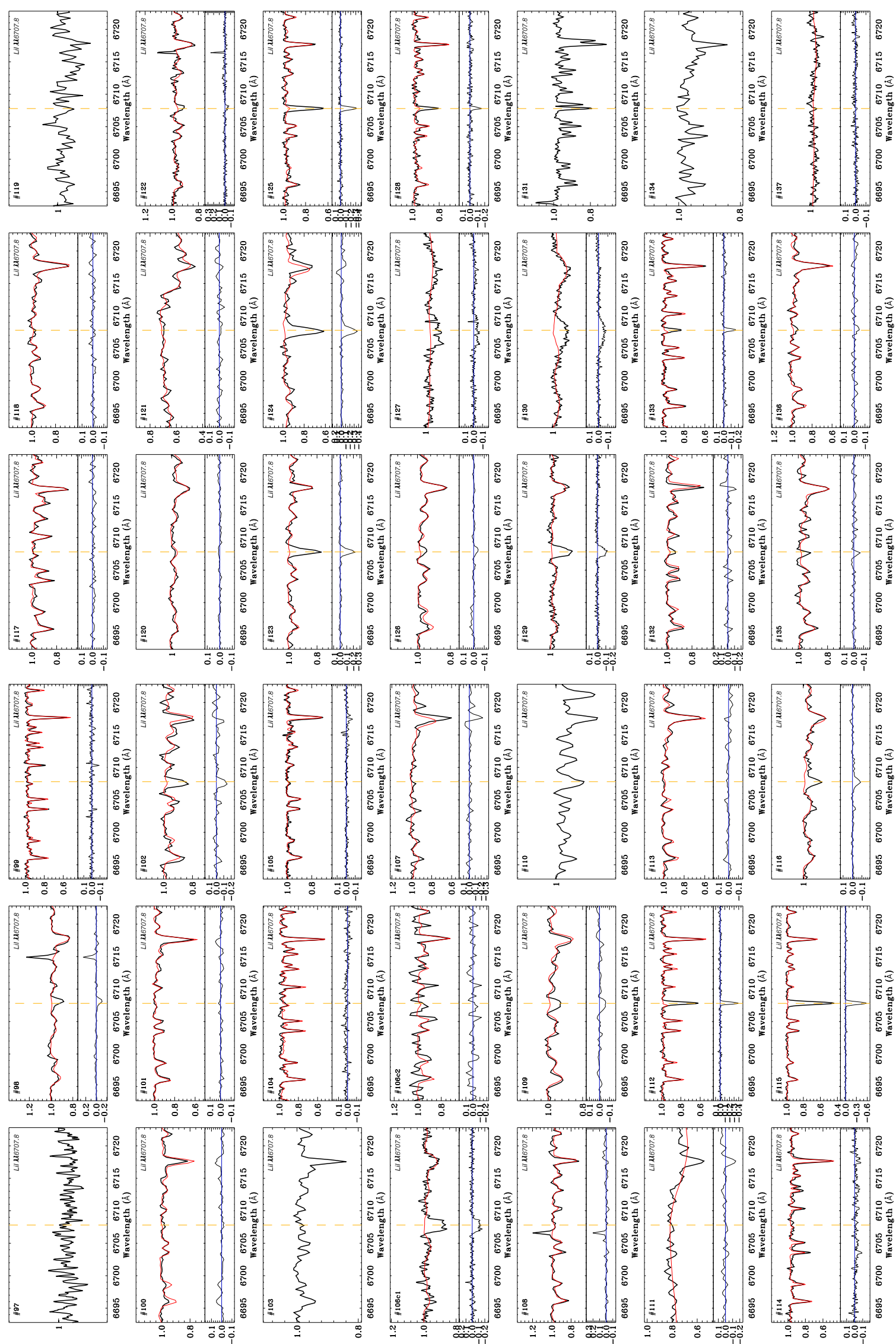

Fig. E.2. continued. 


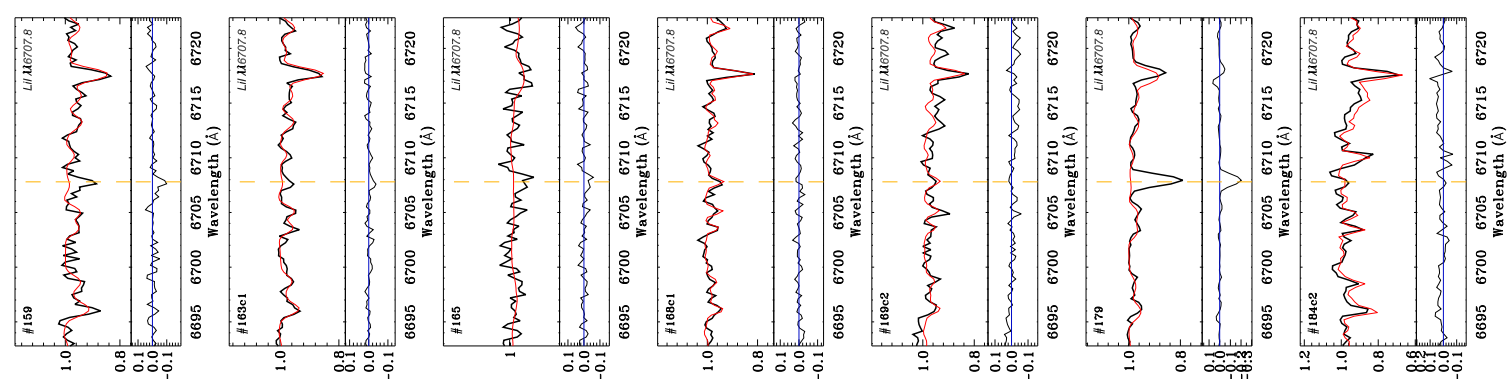

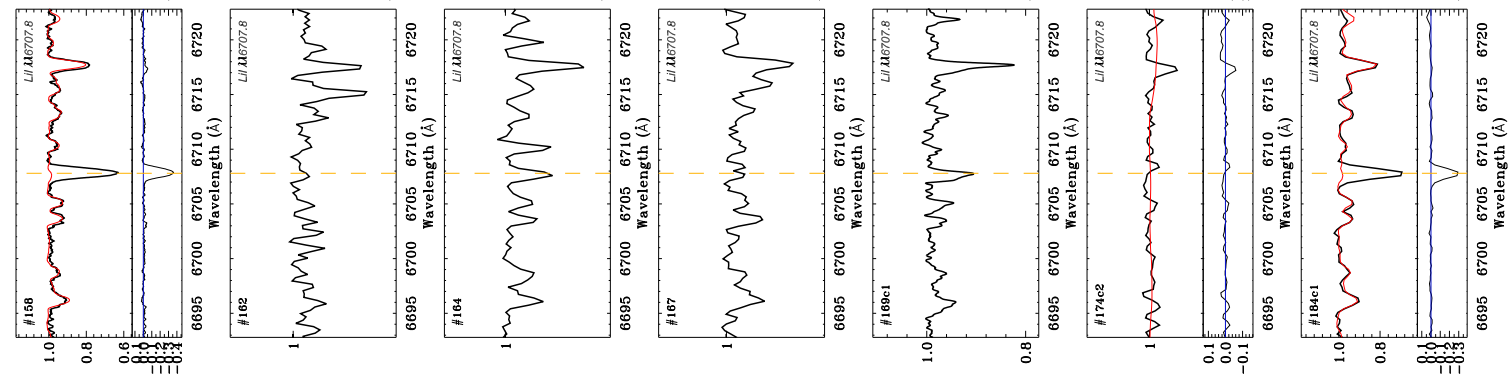
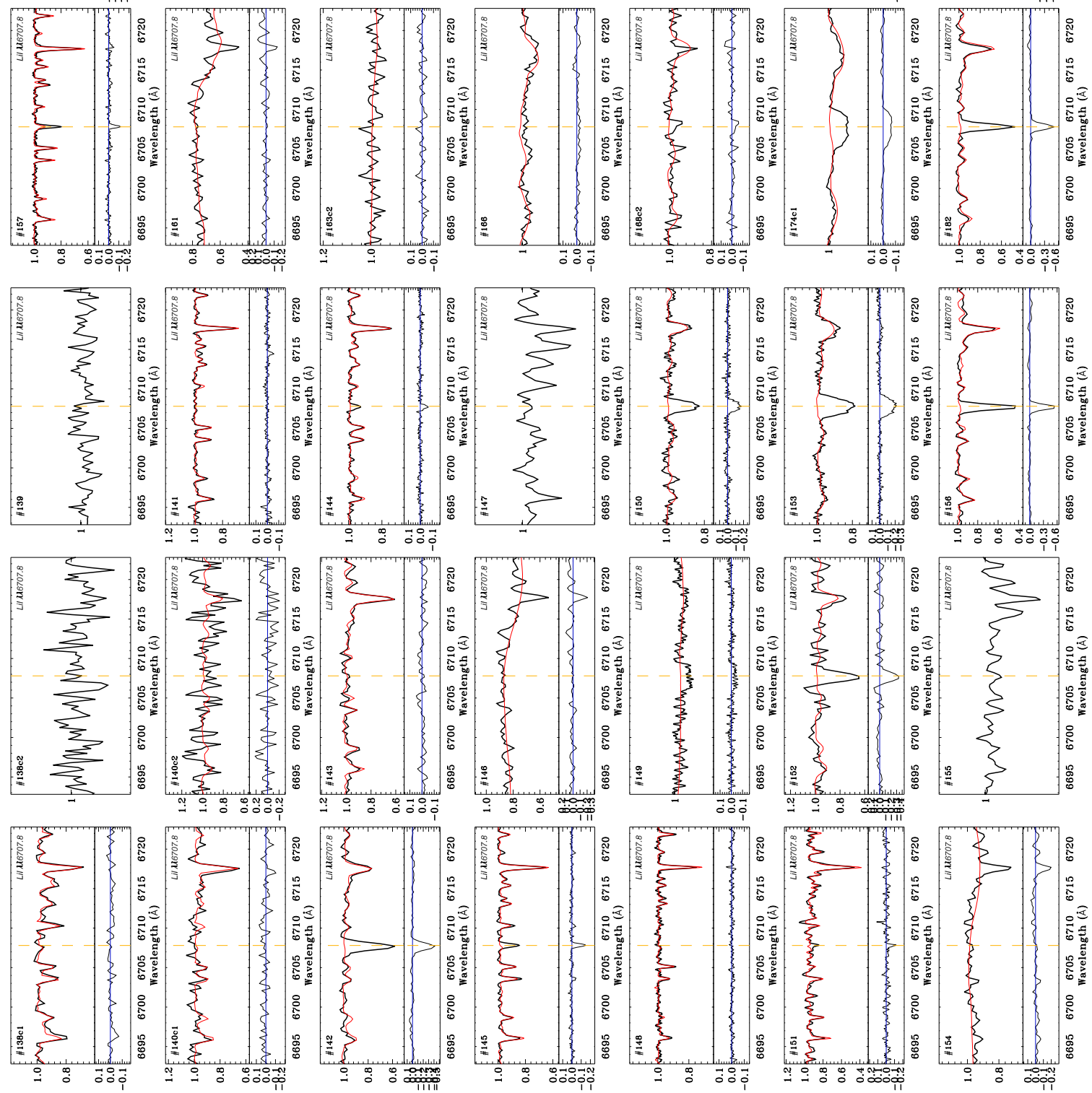

Fig. E.2. continued. 
A. Klutsch et al.: Properties of the young stellar association in Cepheus
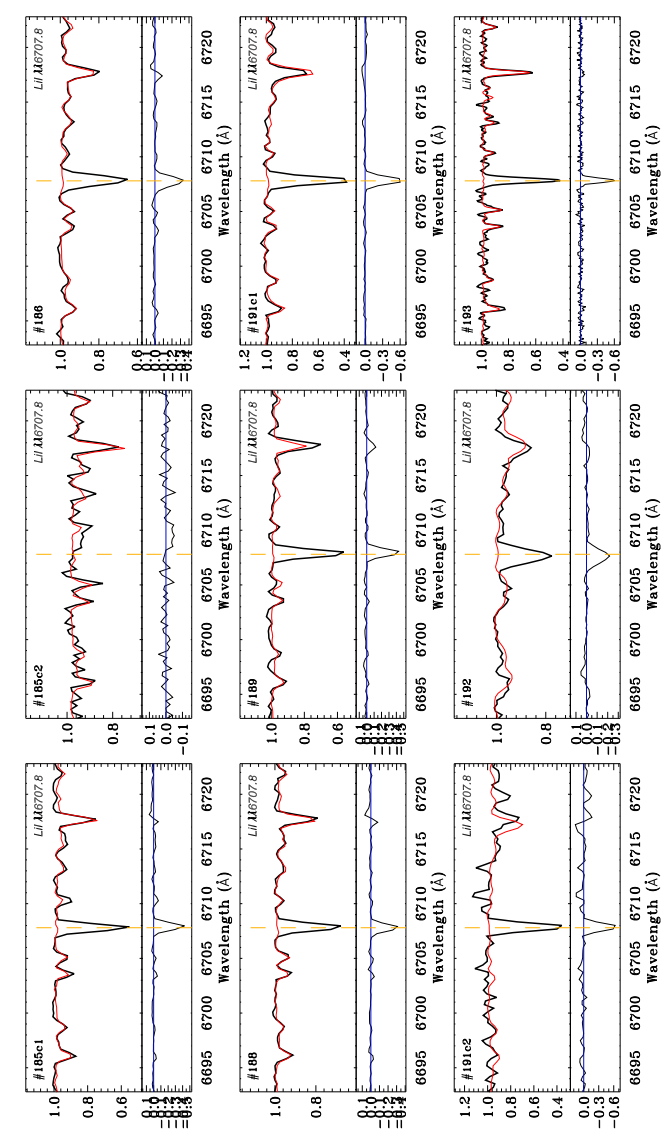

Fig. E.2. continued. 